\title{
Vascular risk levels affect the predictive value of platelet reactivity for the occurrence of MACE in patients on clopidogrel: IPD meta-analysis
}

\begin{tabular}{|c|c|}
\hline Journal: & Thrombosis and Haemostasis \\
\hline Manuscript ID & Draft \\
\hline Manuscript Type: & Original Article: Atherosclerosis and Ischaemic Disease \\
\hline Category: & Clinical Studies \\
\hline Date Submitted by the Author: & $\mathrm{n} / \mathrm{a}$ \\
\hline Complete List of Authors: & $\begin{array}{l}\text { Reny, Jean-Luc; Geneva University Hospitals, Département de médecine } \\
\text { interne, de réhabilitation et de gériatrie } \\
\text { Fontana, Pierre; Geneva University Hospitals, } \\
\text { Hochholzer, Willibald; Freiburg-Bad Krozingen, Klinik, } \\
\text { Neumann, Franz-Josef; Universitaets-Herzzentrum Bad Krozingen, Klinik } \\
\text { fuer Kardiologie und Angiologie II } \\
\text { ten Berg, Jurrien; St. Antonius Hospital, } \\
\text { Janssen, Paul; St. Antonius Hospital, Cardiology } \\
\text { Geisler, Tobias; University Hospital Tübingen, } \\
\text { Gawaz, Meinrad; Eberhard-Karls-Universitaet Tuebingen, Medizinische } \\
\text { Klinik III, Kardiologie } \\
\text { Marcucci, Rossella; University of Florence, Medical and Surgical Critical } \\
\text { Care Medicine } \\
\text { Gori, Anna Maria; University of Florence, Medical and Surgical Critical Care } \\
\text { Medicine, } \\
\text { Cuisset, Thomas; CHU Timone Unité, Inserm UMR 626, } \\
\text { Alessi, Marie-Christine; CHU Timone Unité, Inserm UMR 626, } \\
\text { Berdagué, Philippe; CH Béziers, Cardiology } \\
\text { Gurbel, Paul; Sinai Hospital of Baltimore, } \\
\text { Yong, Gerald; Maryland Royal Perth Hospital, } \\
\text { Angiolillo, Dominick; University of Florida College of Medicine-Jacksonville, } \\
\text { Division of Cardiology } \\
\text { Aradi, Dániel; Heart Institute, University of Pécs, Departement of } \\
\text { Interventional Cardiology; } \\
\text { Beigel, Roy; Sheba Medical Center, Leviev Heart Center } \\
\text { Campo, Gianluca; U.O. Cardiologia, AOU S.Anna Ferrara, Medicina e Clinica } \\
\text { Sperimentale } \\
\text { Combescure, Christophe; University Hospital of Geneva and University of } \\
\text { Geneva, Division of clinical epidemiology }\end{array}$ \\
\hline Keywords: & Antiplatelet agents, Metaanalysis, Ischaemic heart disease \\
\hline
\end{tabular}




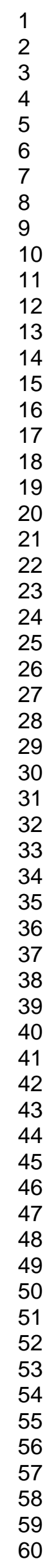

SCHOLARONE $^{\text {'m }}$

Manuscripts 
1 Title: Vascular risk levels affect the predictive value of platelet reactivity for the occurrence of

2 major adverse cardiovascular events in patients on clopidogrel: Systematic review and

collaborative meta-analysis of individual patient data

Running head: Vascular risk, platelet reactivity, and prognosis

6

\author{
Authors: \\ Jean-Luc Reny; Pierre Fontana; Willibald Hochholzer; Franz Josef Neumann; Jurriën ten \\ Berg; Paul W.Janssen; Tobias Geisler; Meinrad Gawaz; Rossella Marcucci; Anna-Maria Gori; \\ Thomas Cuisset; Marie-Christine Alessi; Philippe Berdagué; Paul A. Gurbel; Gerald Yong; \\ Dominick J. Angiolillo; Daniel Aradi; Roy Beigel; Gianluca Campo; and Christophe \\ Combescure.
}

\section{Authors' affiliations:}

Geneva Platelet Group, School of Medicine, University of Geneva, Geneva, Switzerland (Dr. Reny, Dr. Fontana); Division of Internal Medicine and Rehabilitation, Trois-Chêne Hospital, Geneva University Hospitals, Geneva, Switzerland (Dr. Reny); Division of Angiology and Haemostasis, Geneva University Hospitals, Geneva, Switzerland (Dr. Fontana); Universitaets Herzzentrum Freiburg-Bad Krozingen, Klinik für Kardiologie und Angiologie II, Bad Krozingen, Germany (Dr. Hochholzer, Dr. Neumann); St Antonius Hospital, Nieuwegein, the Netherlands (Dr. ten Berg, Dr. Janssen); University Hospital Tübingen, Department of cardiology, Tübingen, Germany (Dr. Geisler, Dr. Gawaz) Center for atherothrombotic diseases, Dept Experimental and Clinical Medicine, University of Florence, Azienda Ospedaliero Universitaria Careggi, Florence, Italy (Dr. Marcucci, Ms Gori); Cardiologie Interventionnelle, CHU Timone Unité Inserm UMR 626, Faculté de Médecine Marseille, France (Dr Cuisset); UMR Inserm 1062, inra 1260, université Aix-Marseille, France (Dr Alessi); Division of Cardiology, Beziers Hospital, Béziers, France (Dr Berdagué); Sinai Center for Thrombosis Research, Sinai Hospital of Baltimore, Baltimore, USA (Dr Gurbel); Maryland Royal Perth Hospital, Australia (Dr. Yong); Division of Cardiology, University of Florida College of Medicine, Jacksonville, Florida, USA (Dr. Angiolillo); Department of Cardiology, Heart Center Balatonfüred, Hungary (Dr. Aradi); The Leviev Heart Center, Sheba Medical Center, Tel-Hashomer, and the Sackler Faculty of Medicine, Tel Aviv University, TelAviv, Israel (Dr. Beigel); Cardiovascular Institute, Azienda Ospedaliera Universitaria di Ferrara, Cona (FE), Italy (Dr Campo); Division of Clinical Epidemiology, University Hospitals of Geneva, Geneva, Switzerland (Dr. Combescure), Center of Clinical Research, Faculty of Medicine, University of Geneva, Geneva, Switzerland (Dr. Combescure)

\section{Corresponding author:}

Jean-Luc Reny

Division of Internal Medicine and Rehabilitation

Trois-Chêne Hospital, Geneva University Hospitals

Ch. du Pont-Bochet 3

$\mathrm{CH}-1226$ Thônex-Geneva

Switzerland

Phone: +41-22-305 6537

Fax: +41-22-305 6115

E-mail: jean-luc.reny@hcuge.ch 


\section{Abstract}

50 Prior studies have shown an association between high on-clopidogrel platelet reactivity (PR)

51 and the risk of major adverse cardiovascular events (MACE). However, large intervention

52 trials on PR-tailored treatments have been neutral. The role and usefulness of PR with

53 regard to levels of cardiovascular risk are unclear. We undertook a systematic review and

54 meta-analysis of individual patient data on MACE outcomes (acute coronary syndromes

55 (ACS), ischemic strokes, and vascular deaths) in relation to PR and its interaction with

56 cardiovascular risk levels. PR was determined using ADP-induced light transmission

57 aggregometry with a primary concentration of $20 \mu \mathrm{M}$ ADP. Thirteen prospective studies

58 totaled 6,478 clopidogrel-treated patients who experienced 421 MACE (6.5\%) during a

59 median follow-up of 12 months. The strength of the association between the risk of MACE

60 and PR increased significantly $(p=0.04)$ with the number of risk factors present (age $>75$

61 years, ACS at inclusion, diabetes, and hypertension). No association was detected in

62 patients with no risk factor $(p=0.48)$. In patients presenting one risk factor, only high-PR was

63 associated with an increased risk of MACE (HR 3.2, $p=0.001)$. In patients presenting $\geq 2$ risk

64 factors, the increase of risk started from medium-PR (medium-PR: HR=2.9, $p=0.0004$; high-

65 PR: HR=3.7, p=0.0003). PR allowed the reclassification of $44 \%$ of the total population to a

66 different risk level for the outcome of MACE, mostly in intermediate or high risk patients.

67 In conclusion, the magnitude of the association between PR and MACE risk is strongly

68 dependent on the level of cardiovascular risk faced by patients on clopidogrel.

Keywords: clopidogrel, drug response, platelets, cardiovascular diseases, ischemic events. 


\section{Introduction}

Atherosclerotic diseases account for more than $40 \%$ of deaths in Western countries, and antiplatelet therapy is a major preventive strategy in this setting(1). Clopidogrel, a $P 2 Y_{12}$ receptor blocker, inhibits the activation of platelets by adenosine diphosphate (ADP), and is widely prescribed for secondary prevention in patients with atherosclerotic diseases. When combined with aspirin, clopidogrel is particularly effective in patients with acute coronary syndromes (ACS)(2), and has proved superior to aspirin alone in several other large randomised controlled trials. The pharmacodynamic response to clopidogrel shows a wide inter-individual variability $(3,4)$. Numerous cohort studies, often performed on patients with acute coronary syndrome (ACS) and/or undergoing percutaneous coronary interventions $(\mathrm{PCl})$, have shown an association between high on-treatment platelet reactivity $(\mathrm{PR})$ and the risk of recurrent major adverse cardiovascular events (MACE)(5-7). However, recent studies in cohorts of stable cardiovascular outpatients $(8,9)$ or in medically managed ACS patients(10) failed to confirm these results. Several randomized trials aimed at reducing the recurrence of ischemic events have compared standard clopidogrel treatment to a $\mathrm{P}_{2} \mathrm{Y}_{12^{-}}$ inhibitor strategy tailored according to the presence of high PR. Although initial small trials were promising $(11,12)$ more recent larger trials showed no benefit from adjusting clopidogrel doses or switching to prasugrel based on PR testing in low-risk coronary patients undergoing $\mathrm{PCl}(13,14)$. These contrasting results, both from observational studies and randomized intervention trials, may be explained by different patient characteristics including the level of risk, but to date few data substantiate these hypotheses. We previously showed, in a studylevel meta-analysis, that the risk of recurrent MACE associated with high PR was greater in studies using Gpllb/llla inhibitors (a marker of high-risk patients) than in studies which did not(7). Another meta-regression from a study-level meta-analysis of randomized trials suggested that the higher the incidence of coronary stent thrombosis in a given study, the larger the net clinical benefit from a PR-tailored strategy(15). Finally, the ADAPT-DES registry of patients undergoing $\mathrm{PCl}$ showed that high $\mathrm{PR}$ was predictive of stent thrombosis mostly in ACS patients, but there was no interaction reported between PR and the presence 
101 of an ACS at inclusion(16). This information suggests the hypothesis that high PR might be

102 more relevant in high-risk populations, but convincing data at the individual level are lacking.

103 To date, the only meta-analysis on individual patient data performed on 6 studies totaling

1043,059 patients assessed with the VerifyNow P2Y12 assay did not explore this hypothesis(17).

105 Similarly, one of the largest and more recent meta-analysis on 8 studies and 4817 patients

106 did not explore this interaction due to the lack of individual data(18). To further investigate

107 this interaction on a larger population we performed a collaborative meta-analysis of

108 individual patient data and focused on the interaction between relevant vascular risk factors

109 and PR, assessed with ADP induced light transmission aggregometry (LTA), in order to

110 better define the risk of MACE. ADP-induced LTA is the assay upon which all P2Y 12 receptor

111 inhibitors have been developed, thus supporting its use in the present meta-analysis. In

112 addition, among several available assays to evaluate PR, LTA is the historical gold standard

113 with which most platelet function assays were compared.

114 


\section{Methods}

116 Data sources

117 Literature review, confined to articles in English(19), was based on electronic databases

118 (Medline, Embase, Web of Science, Cochrane Central Register of Controlled Trials) and

119 abstracts from major international meetings held from 2010-2013 (ISTH, AHA, ACC, ESC).

120 A free-text search was conducted using an 'ADP' and 'aggregation' and 'clopidogrel' key-

121 word combination. Articles were selected on the basis of abstracts, before examination of the

122 full text. Reference lists of selected articles were also hand-searched to identify additional

123 relevant reports. Reviewers (JLR and PF) were not blinded to the journal, authors or

124 institutions in the publications as this has been shown to be unnecessary(20). The electronic

125 database search was last updated on 31 July, 2013. The objective of this individual patients'

126 data meta-analysis was described in a project that was part of French ministry of health's

127 initiative to encourage meta-analyses (PHRC 15-07 to JL Reny "Etudes prospectives sur la

128 réponse biologique au clopidogrel et évènements ischémiques chez les patients

129 athérothrombotiques : Métaanalyse sur données individuelles et résumées" http://www.plan-

130 alzheimer.gouv.fr/IMG/pdf/Liste des dossiers retenus - 2 mai 2008.pdf ). Protocol in

131 French available upon request.

132

133 Study selection

134 Selected studies met the following criteria: (a) patients were treated with clopidogrel and had 135 symptomatic atherothrombosis (clinical signs related to vascular atherothrombotic lesions);

136 (b) pharmacodynamic response to clopidogrel was evaluated using the maximal aggregation 137 value from LTA on platelet-rich plasma with 20 , 10 , or $5 \mu \mathrm{M}$ ADP as an agonist; (c) LTA was 138 performed remote from platelet function interfering drugs such as Gpllb/llla inhibitors; (d) 139 patients were prospectively monitored for MACE for at least 30 days, defined using at least

140 one of the following items: acute coronary syndrome (unstable angina, myocardial infarction

141 with/without ST segment elevation), ischemic stroke (acute neurological deficit due to a

142 cerebral infarction), and vascular death; (e) studies involved either a prospective cohort or a 
143 randomised therapeutic trial, but one in which treatment was allocated independently of the

144 response to clopidogrel. When studies were suspected of including the same patients, the

145 authors were asked to provide data from the largest possible number of independent patients.

146

147 Data extraction

148 The corresponding authors or principal investigators of eligible studies were contacted and

149 asked to participate in the CLOpidogrel and Vascular ISchemic events - Individual Patient

150 Data (CLOVIS-IPD) meta-analysis group. Investigators provided individual data on: the

151 qualifying cardiovascular condition and clinical setting at inclusion (ACS or stable disease);

152 MACE and date of occurrence during follow-up; platelet reactivity (PR) with ADP 20, 10,

153 and/or $5 \mu \mathrm{M}$ and its timing relative to loading dose of clopidogrel; age, gender, height, and

154 weight; current smoking status, diabetes, hypercholesterolemia, and hypertension; left

155 ventricular ejection fraction; platelet count; PCl; use of Gpllb/llla inhibitors and timing;

156 concomitant medications; and bleeding events and timing during follow-up. Data were

157 checked for completeness and consistency with published reports. Any discrepancies were

158 resolved with the corresponding authors. After format harmonization, data were compiled for

159 statistical analysis. All studies were approved by their respective institutional review boards.

160

161 Quality assessment of studies

162 A new quality assessment tool for prognostic studies called PROBAST (see

163 Acknowledgements) was used to estimate risks of bias and concerns about applicability. As

164 PROBAST is not customized for meta-analyses of individual patient data, items were

165 adapted accordingly. Based on the present study's list of relevant criteria, risks of bias, and

166 concerns about applicability are rated as low, unclear, or high. Supplemental Figure 1 shows

167 the list of criteria.

168

169 Primary outcomes and measures 
170 The primary clinical outcome was the occurrence of MACE, as defined above (see Study

171 selection (d)). The primary biological outcome was maximal aggregation with $20 \mu \mathrm{M} A D P$, as

172 it is a better concentration for analyzing the effects of clopidogrel than lower ones. PR was

173 categorized in three strata. The higher cut-offs were selected on the basis of previously

174 published cut-offs (59\% to $64 \%$ for $20 \mu \mathrm{M}$ ADP, and $43 \%$ to $46 \%$ for $5 \mu \mathrm{M}$ ADP)(21), and to

175 keep relatively balanced numbers of patients in each PR categories. Three pre-specified

176 categories allowed a better description of the dose-dependent effects of PR on the risk of

177 MACE compared to the usual dichotomous high and low PR categorization. Three categories

178 were also chosen to better parallel the analysis with a therapeutic PR window that has been

179 associated with optimal net clinical benefit(22). A surrogate for the level of cardiovascular

180 risk was defined as the number of factors with homogeneous definitions across studies, and

181 these were markers of MACE in the meta-analysis. The factors were selected from among

182 age, diabetes, hypertension, smoking, hypercholesterolemia, and the presence of an ACS at

183 inclusion (as defined in study selection (d)), and were all provided at the time of inclusion and

184 PR testing.

185

186 Statistical analysis

187 MACE-free survival curves were derived from individual patient data using the Kaplan-Meier

188 estimator; curves were compared using log-rank tests stratified by study. Associations

189 between conventional risk factors, PR strata, and risk of MACE were analyzed using

190 multivariate, mixed-effect Cox models. The amount of heterogeneity was assessed by the

191 size of the random effects $\left(\mathrm{Tau}^{2}\right)$ which is an estimate of the between study variability(23).

192 The presence of heterogeneity was tested by comparing models with and without random

193 effects (likelihood ratio test). The interactions between the level of risk and PR strata were

194 tested. MACE-free survival according to PR, as a continuous variable, was assessed using

195 the R package prodlim using the symmetrical nearest neighborhoods method.(24) Sensitivity

196 analyses were conducted to check the robustness of the findings with respect to: the risks of

197 bias and concerns about the applicability of studies; the definition of MACE, including target 
198 vessel revascularization or $\mathrm{PCl}$ at inclusion, and; the influence of a given specific study. The

199 net reclassification index (NRI) for survival data(25) was computed to quantify the

200 contribution of PR testing for the prediction of the 6-month risk of MACE in patients with

201 increasing numbers of traditional risk factors. The event and non-event continuous NRIs

202 were reported. Potential publication bias was checked for. P-values below 0.05 were

203 considered significant and all tests were two-sided. Published guidelines for meta-analysis of

204 observational studies in epidemiology (MOOSE) and their reporting(26) were followed.

205 Details on statistical methods are given in the online data supplement.

206

207 Results

208 Characteristics of included studies

209 The Figure 1 flow-chart details how 13 of 20 qualifying studies were included, totaling 6,478

210 patients $(8,27-38)$. Table 1 shows their characteristics. Data on body mass index,

211 concomitant medications, left ventricular ejection fraction, or the occurrence of target and

212 non-target vessel revascularization during follow-up were only available in some studies. All

213 studies provided individual data allowing a homogeneous definition of MACE, current

214 smoking status, ACS, diabetes (fasting plasma glucose $\geq 7.0 \mathrm{mmol} / \mathrm{l}$, 2-h plasma glucose $\geq$

$21511.1 \mathrm{mmol} / \mathrm{l}$ after $75 \mathrm{~g}$ oral glucose load or background therapy for diabetes), and

216 hypertension (systolic blood pressure $\geq 140 \mathrm{mmHg}$ or diastolic blood pressure $\geq 90 \mathrm{mmHg}$ or

217 a documented history of hypertension). Hypercholesterolemia was not defined in a

218 homogeneous fashion across studies and plasma LDL-cholesterol levels were not available

219 for more than 2,000 patients. Overall, risks of bias and concerns about applicability were low

220 (online data supplement further details study characteristics, bias, and applicability).

221 Information on bleeding was limited to five studies, with only 67 major and 20

222 moderate/minor bleedings.

223

224 MACE and level of risk 
225 Overall, 421 MACE occurred in 6,478 patients $(6.5 \%)$, the majority being ACS $(n=383)$.

226 There were 83 stent thromboses, including 79 definite or probable and four possible ones, all

227 included in the composite outcome of MACE. The MACE-free survival rate across the

228 different studies at the end of follow-up ranged from $77.4 \%$ to $97.3 \%$. In a multivariate

229 analysis, four factors were found relevant to determining patients' levels of risk: age greater

230 than 75 years, diabetes, ACS at inclusion, and hypertension (Table 2). The number of these

231 factors was used as a surrogate for the individual risk of MACE. Patients with none of these

232 factors were classified 'low-risk', patients with one factor 'intermediate-risk', and patients with

233 two or more factors 'high-risk' (global p-value $<0.0001$ for the trend).

234

235 MACE and PR

236 Nine studies ( $n=4,438$ patients) performed LTA using $20 \mu$ MDP, four studies $(n=2,144$

237 patients) used $10 \mu \mathrm{M}$ ADP, and eight studies ( $n=3,317$ patients) used $5 \mu$ M ADP. Figure 2

238 shows the MACE-free survival curves by category of ADP concentration. Risk of MACE

239 increased significantly with PR with $20 \mu \mathrm{M}$ ADP, $10 \mu \mathrm{M}$ ADP, and $5 \mu \mathrm{M}$ ADP.

240 With adjustment, high PR was still significantly associated with an increased risk of MACE

241 (Table 3). However, for PR evaluated using $10 \mu \mathrm{M}$ ADP, risk only increased for the highest

242 PR category, corresponding to LTA values greater than $60 \%$.

243

244 Interaction between risk level and PR for the outcome of MACE

246 Platelet reactivity assessed with $20 \mu \mathrm{M}$ ADP. Patients with none of the four risk factors

247 showed no significantly increased risk associated with PR, while for patients with one risk

248 factor only, the higher strata of PR was associated with an increased risk of MACE. Patients

249 with two or more risk factors showed an increased risk of MACE for both the medium and

250 higher strata of PR. (Figure 3). In a Cox model, the interaction between PR strata and the

251 risk level was statistically significant $(p=0.04)$. The corresponding hazard ratios $(\mathrm{HRs})$ are

252 shown in Figure 3. Heterogeneity was not detected for the overall interaction $(p=0.81)$, as 
253 well as when it was restricted to each risk level category (intermediate versus low risk level, $254 \mathrm{p}=0.45$, and high versus low risk level, $\mathrm{p}=0.90$ ). Additional results on heterogeneity are 255 provided in the supplemental material. Figure 4A shows that PR, when analyzed in a 256 continuous fashion, barely affects the risk of MACE at 6 months in patients with no risk 257 factors: the risk is close to $2 \%$ at six months, irrespective of the level of platelet reactivity. 258 Conversely, patients with one risk factor and an overall $4.1 \%$ risk of MACE at six months 259 have in fact a $2 \%$ risk of MACE when they have a low PR, or a $6 \%$ risk of MACE when they 260 have a high PR (Figure 4B). Similarly, patients with two or more risk factors and an overall $2616 \%$ risk of MACE at six months can indeed have a $2 \%$ risk of MACE when they have a low 262 PR (Figure 4C). The reclassification of the 6-month risk of MACE, according to the three 263 categories of platelet reactivity, in patients with no, one and two or more risk factors, is 264 shown in Table 4. Overall, PR allowed the reclassification of $44 \%$ of the total population 265 (1837/4193 patients) included in a 6-month follow-up to a different level, mostly in patients 266 originally identified as intermediate or high risk on the basis of the number of risk factors only.

267 In patients experiencing MACE in the first 6 months of follow-up, the risk predicted by the 268 combination of PR and risk factors was on average increased compared with the risk 269 predicted from risk factors only: the continuous event net reclassification index (NRI) was $270 \quad 0.39(95 \% \mathrm{Cl} 0.23$ to 0.62$)$. Conversely, in patients free of MACE at 6 months, the measure of 271 PR did not modify the predicted risk: the continuous non-event $\mathrm{NRI}$ was $0.01(95 \% \mathrm{Cl}-0.16$ to 272 0.09). The overall NRI was $0.39(95 \% \mathrm{Cl} 0.22$ to 0.57$)$.

274 Platelet reactivity assessed with $10 \mu \mathrm{M}$ ADP. A total of only five low-risk patients in four 275 studies performing $10 \mu \mathrm{M}$ ADP LTA to assess PR precluded an analysis of this low-risk 276 group. Furthermore, the surrogate for risk level failed to demonstrate an association with the 277 observed risk of MACE in these studies. Figure 4B shows that the risk of MACE increased in 278 both intermediate- and high-risk patients for PR values above $40 \%$, without any obvious 279 relation with the level of risk. 
$281 \quad$ Platelet reactivity assessed with $5 \mu \mathrm{M}$ ADP. The direction of interaction between PR using 5

$282 \mu \mathrm{M}$ ADP and the risk level was similar to that observed for PR using $20 \mu \mathrm{M}$ ADP, even

283 though overall interaction did not reach the significance level $(p=0.17)$. Of note there were

284980 fewer patients in the studies performing $5 \mu \mathrm{M}$ ADP than in those using $20 \mu \mathrm{M}$ ADP. The

285 increased risk of MACE as PR increases is indeed similar for intermediate- and high-risk

286 patients; for low-risk patients PR is not associated with a MACE outcome (online data

287 supplement). Heterogeneity was not detected for the overall interaction $(p=0.19)$. Figure $4 \mathrm{C}$

288 shows that the risk of MACE was unaffected by PR in low-risk patients while it increased for

289 PR values above $30 \%$ in intermediate-risk patients and for PR values above $10 \%-20 \%$ in

290 high-risk patients.

291

292 Sensitivity analyses

293 Sensitivity analyses were performed for PR using $20 \mu \mathrm{M}$ ADP to assess: the robustness of

294 the association between PR and risk of MACE and its interaction with the level of

295 cardiovascular risk; the robustness of the results in the population of $\mathrm{PCl}$ patients and when

296 target vessel revascularization is added to the composite outcome. All analyses showed that

297 the sizes of the effects remained similar, and whilst in some instances the statistical

298 significance of the interactions could be lost, there was no impact on their magnitudes

299 (supplemental Tables 1 and 2). Notably, when PR was categorized in quartiles (20 $\mu$ M ADP

300 maximal aggregation quartiles $=0 \%-38.1 \%, 38.2 \%-51.3 \% .51 .4 \%-63.0 \%, 63.1 \%-100 \%$ )

301 the interaction between PR and the number of risk factors remained significant $(p=0.01)$.

302 When restricted to the population of 3,564 patients treated with $\mathrm{PCl}$ and tested using $20 \mu \mathrm{M}$

303 ADP the interaction was of similar magnitude but no longer significant (supplemental Table

$3043)$.

305

306 Publication and availability biases

307 A check for potential publication bias was made for PR using $20 \mu \mathrm{M}$ ADP, on which the main 308 analyses were performed. A funnel plot was obtained by representing the HR of PR using 20 
$309 \mu \mathrm{M}$ ADP and the standard error, assessed in each separate study (supplemental Figure 4).

310 Two studies with a negative association between PR using $20 \mu \mathrm{M}$ ADP and the risk of MACE

311 (with small sample sizes) were detected as missing using the 'trim and fill' method for making

312 the funnel plot symmetrical. When these missing studies were added, the pooled HR was not

313 significantly modified. These findings suggested that the publication bias in our meta-analysis

314 was minor.

315 Seven qualifying studies could not provide individual patient data. It is of note that in five of

316 these, the relation between clopidogrel non-response and ischemic events was not a study

317 objective (pharmacokinetic-pharmacodynamic studies or randomized trials of different

318 clopidogrel loading doses). The two remaining studies ( $n=101$ and 111 patients) were

319 specifically interested in the prognostic value of PR for MACE.

320 


\section{Discussion}

322 In the present meta-analysis of individual patient data conducted in a representative panel of 323 clopidogrel-treated patients we demonstrated that the association between PR and the risk of 324 MACE depended strongly on the level of cardiovascular risk. When using $20 \mu \mathrm{M}$ ADP, the 325 most commonly used concentration in LTA, the risk of MACE associated with PR increased 326 with the level of cardiovascular risk. Indeed, PR did not affect the risk of MACE in patients 327 presenting no risk factors, however it gradually increased the risk of MACE as the number of 328 cardiovascular risk factors increased, reaching a 3.7 times greater risk in high-risk patients 329 with a high PR. The measure of PR with $20 \mu \mathrm{M}$ ADP, in addition to risk factors, modified the 330 interpretation of the 6 -month risk of MACE in $44 \%$ of patients, mainly in patients with at least

331 one risk factor.

332 Interestingly, smoking and hypercholesterolemia were not associated with the outcome of 333 MACE and were not included in the analysis of the interaction between PR and risk factors. 334 In randomized controlled trials, the benefit of clopidogrel in reducing the incidence of MACE 335 is primarily seen in smokers, with little benefit to non-smokers(39). With regard to the cohort 336 studies of clopidogrel-treated patients included in this meta-analysis, this differential effect

337 suggests that the increased risk of MACE related to smoking is offset by the benefit 338 clopidogrel provides to smokers; it thereby weakens any possible analysis of the interaction 339 between smoking and PR for outcomes of MACE. Regarding hypercholesterolemia, this 340 conventional risk factor is likely to be confounded by indications for statin treatment. Indeed, 341 in the ADAPT-DES registry(16) hyperlipidemia was protective against mortality with a $342 \mathrm{HR}=0.60(0.41-0.86)$ and was not prognostic of MACE in post-ACS patients with optimal 343 medical therapy(40). In addition, hypercholesterolemia was not homogeneously defined 344 across the studies in the present meta-analysis and other markers, such as plasma LDL345 cholesterol levels, were not widely available.

346 When PR was evaluated using $5 \mu \mathrm{M}$ ADP, its interaction with the level of cardiovascular risk

347 for the prediction of MACE was of a similar magnitude, although non-significant. These

348 findings may reflect the lower number of patients available in studies using $5 \mu \mathrm{M}$ ADP, and a 
349 corresponding loss of power. Moreover, it was previously shown that ADP-induced platelet

350 aggregation in citrated plasma was dependent on the artifactual generation of TxA2 that was 351 modulated by aspirin, at least at lower ADP concentrations(41). This may be associated with 352 an additional background noise in which the interaction between the identified risk factors 353 and PR to predict MACE is blurred, as seen with the lowest $5 \mu \mathrm{M}$ ADP concentrations and 354 partially also with the intermediate $10 \mu \mathrm{M}$ ADP concentrations. Only four of the studies 355 analysed used $10 \mu \mathrm{M}$ ADP, and two of these had a follow-up limited to 30 days; with only 356124 MACEs during follow-up, this accounts for a limitation in power to reliably study 357 interactions. Overall, the concentration of ADP used is of limited significance since the 358 influence of risk factors appears in all three ADP concentration groups (table 3 and figure 2).

359 Which laboratory assay and which platelet agonist concentration are best suited for the 360 clinical evaluation of platelet function is the matter of some debate. ADP-induced LTA is 361 highly reproducible within a given laboratory, but its lack of standardization across studies 362 may have slightly weakened the positive findings or lower the level of significance for the 363 interactions found in the present meta-analysis. Of note, the present meta-analysis does not 364 aim to promote the use of LTA to tailor antiplatelet therapy but it rather relied on a historical 365 gold standard in platelet function testing to evidence an interaction with patients' 366 characteristics that should be considered for a tailored approach. The point-of care 367 VerifyNow P2Y 12 assay, used in several intervention trials, correlates well with ADP-induced $368 \operatorname{LTA}(42,43)$ and we speculate that the main findings of the present meta-analysis would 369 have been similar, had PR been evaluated using the VerifyNow P2Y ${ }_{12}$ assay.

370 Several intervention trials have compared conventional clopidogrel treatment to an 371 antiplatelet strategy tailored according to PR. Early, small randomized trials(11, 12) that 372 utilized vasodilator-stimulated phosphoprotein phosphorylation level measurement to indicate $373 \mathrm{P}_{2} \mathrm{Y}_{12}$ receptor reactivity, showed a protective effect for repeat $600 \mathrm{mg}$ clopidogrel loading 374 doses in ACS patients prior to $\mathrm{PCl}$. However, recent larger trials utilizing the VerifyNow $\mathrm{P}_{2} \mathrm{Y}_{12}$ 375 assay were negative. Indeed, the GRAVITAS(13) and ARCTIC(14) studies failed to show the 376 benefit of a PR-tailored antiplatelet strategy after PCl. Various limitations of these trials were 
377 addressed in a recent consensus publication(22). The event rate of the GRAVITAS study

378 was low compared to the one used for power calculation, and the antiplatelet effect of the

379 high-dose regimen may have been suboptimal as it reduced the prevalence of high PR by

380 only $22 \%$. Similarly, the ARTIC study population was also at a low absolute risk of

381 subsequent cardiovascular events because the prevalence of ACS patients was low, and the

382 composite endpoint also included other events that may not be related to platelet function.

383 The interaction of PR and the number of risk factors, as identified in the present meta-

384 analysis, substantiates the hypothesis that the risk associated with high PR was not clinically

385 relevant in low-risk patients, and that any measure aiming to lower PR is unlikely to lead to a

386 beneficial reduction of MACE for these low-risk patients. Based on these observations we

387 speculate that higher risk patients are more likely to benefit from a therapy tailored to their

388 initial PR. This may explain why early interventions designed to efficiently blunt high PR in

389 ACS patients with multiple conventional risk factors translated into a reduction of MACE(11,

390 12, 22).

391 In the current new antiplatelet era, prasugrel and ticagrelor have a major part to play in the

392 management of ACS, leaving clopidogrel as an alternative for patients with high bleeding risk.

393 However, a recent cost-effectiveness analysis for six European perspectives showed that the

394 universal use of newer $\mathrm{P}_{2} \mathrm{Y}_{12}$ inhibitors for ACS patients is probably not as cost-effective as

395 strategies based on $\mathrm{PR}(44)$. It should also be kept in mind that ticagrelor and prasugrel

396 increase the risk of bleeding and that a therapeutic medium-PR window is associated with

397 optimal net clinical benefit(22). The net benefits of newer $\mathrm{P}_{2} \mathrm{Y}_{12}$ inhibitors could also probably

398 be improved not only by testing for PR, but also by incorporating patient risk levels in the

399 decision-making process. Although ongoing trials on tailored $\mathrm{P}_{2} \mathrm{Y}_{12}$ strategies, including

400 TROPICAL-ACS (ClinicalTrials.gov identifier: NCT01959451) and ANTARCTIC(45) partly

401 include this concept of risk levels, further efforts in this direction are needed.

402 This meta-analysis has several strengths, such as the good overall quality of the studies

403 included, as assessed using a quality tool specifically adapted to prognostic studies. The

404 availability of individual patient data allowed a reliable evaluation of the risk associated with 
405 PR and of the interaction with vascular risk factors. Readily available risk factors relevant to a 406 secondary prevention population were thus identified. The consistency of results across the 407 different ADP concentrations used in the different studies to assess PR, as well as the 408 sensitivity analyses, indicated that the results were robust.

409 Despite the advantages related to the availability of individual patient data, this meta-analysis 410 also had some limitations, including a low proportion of women (25\%). This did not allow a 411 stratification of the analyses by gender, as is usually the case in risk assessment tools such 412 the European SCORE or the Framingham risk score. Indeed, in these latter scores gender is 413 not considered as one of traditional risk factors, but is rather presented in separate charts for 414 women and men. There were incomplete data on concomitant medications or other relevant 415 risk factors such as the left ventricular ejection fraction, cholesterol levels or renal 416 insufficiency. Finally, information on bleeding was limited to five studies and a low number of 417 events, thus precluding a reliable analysis of bleeding events and their relation to PR. 418 In conclusion, high PR in patients on clopidogrel is associated with an increased risk of 419 MACE in patients with vascular risk factors, but not in low-risk patients. These findings 420 suggest that trials on tailored PR treatment strategies should be primarily stratified on the 421 individual vascular risk factors in order to assess a truly personalized approach.

422

423 Funding statement: French ministry of health PHRC 15-07 to JL Reny "Etudes prospectives 424 sur la réponse biologique au clopidogrel et évènements ischémiques chez les patients 425 athérothrombotiques : Métaanalyse sur données individuelles et résumées" (http://www.plan426 alzheimer.gouv.fr/IMG/pdf/Liste des dossiers retenus - 2 mai 2008.pdf ) and the Geneva 427 University Hospitals. Conducted within the Geneva Platelet Group and the division of Clinical 428 Epidemiology. The funders had no role in study design, data collection and analysis, decision 429 to publish, or preparation of the manuscript.

\section{Acknowledgments:}


432 We thank P Whiting for advices on the evaluation of the quality using the PROBAST tool:

433 The PROBAST tool for the assessment of risk of bias and concerns about applicability for 434 prognostic studies was kindly provided by the PROBAST steering group. This tool is still in 435 development and is available from the PROBAST team (www.systematic-reviews.com/probast).

436

\section{Author contributions:}

438 Reny JL, Fontana $\mathrm{P}$, and Combescure $\mathrm{C}$ are guarantors for the study, had full access to the 439 data and take responsibility for the integrity of the data and the accuracy of its analysis 440 Study concept and design: Reny JL, Fontana P, and Combescure C 441 Acquisition of data: Reny JL, Fontana P, Hochholzer W, Neumann FJ, Ten Berg J, Janssen 442 PW, Geisler T, Gawaz M, Marcucci R, Gori AM, Cuisset T, Alessi MC, Berdagué P, Gurbel P, 443 Yong G, Angiolillo D, Aradi D, Beigel R, Campo G.

444 Data management and statistical analysis: Combescure C and Reny JL

445 Drafting and critical revision of the manuscript for important intellectual content:: Reny JL, 446 Fontana P, Hochholzer W, Neumann FJ, Ten Berg J, Janssen P, Geisler T, Gawaz M, 447 Marcucci R, Gori AM, Cuisset T, Alessi MC, Berdagué P, Gurbel P, Yong G, Angiolillo D, 448 Aradi D, Beigel R, Campo G, Combescure C.

449 All authors had full access to all of the data (including statistical reports and tables) in the 450 study and can take responsibility for the integrity of the data.

\section{Conflicts of interest:}

453 Reny JL: payment for lectures by Merck Sharp and Dohme

454 Fontana P: consultancy for Evolva; grants from Evolva and Astra Zeneca, payment for

455 lectures by Bayer, AstraZeneca.

456 Hochholzer W: no conflicts of interest.

457 Neumann FJ: no conflicts of interest

458 Ten Berg J: consultancy for Astra Zeneca, Eli Lilly, Merck, and Daiichi Sankyo

459 Janssen $\mathrm{P}$ : no conflicts of interest 
460 Geisler T: consultancy for Bayer, Medicines company, Eli Lilly, Pfizer, BMS, and Daiichi 461 Sankyo; payments for lectures by Bayer, Medicines company, Eli Lilly, Pfizer, BMS, and 462 Daiichi Sankyo; MSD, Boehringer, Astra Zeneca.

463 Gawaz M: consulting fee for Bayer, Astra Zeneca, MSD. Lilly; consultancy for Boehringer464 Ingelheim

465 Marcucci R: no conflicts of interest

466 Gori AM: no conflicts of interest

467 Cuisset $\mathrm{T}$ : no conflicts of interest

468 Alessi MC: Board membership for Astra Zeneca and Lilly; lectures for Roche;

469 Berdagué $P$ : no conflicts of interest

470 Gurbel P: Served as a consultant for Daiichi Sankyo, Sankyo, Lilly, Bayer, AstraZeneca, 471 Accumetrics, Merck, Medtronic, CSL, and Haemonetics; receiving grants from the National 472 Institutes of Health, Daiichi Sankyo, Lilly, CSL, AstraZeneca, Harvard Clinical Research 473 Institute, Haemonetics, and Duke Clinical Research Institute; receiving payment for lectures, 474 including service on speakers' bureaus, from Lilly, Daiichi Sankyo, and Merck; receiving 475 payment for development of educational presentations from Merck, the Discovery Channel, 476 and Pri-Med; Dr. Gurbel holds stock or stock options in Merck, Medtronic, and Pfizer; and 477 holds patents in the area of personalised antiplatelet therapy and interventional cardiology. 478 Yong G: no conflicts of interest

479 Angiolillo DJ: Received payment as an individual for: a) Consulting fee or honorarium from 480 Bristol-Myers Squibb, Sanofi-Aventis, Eli Lilly, Daiichi Sankyo, Inc., The Medicines Company, 481 AstraZeneca, Merck, Evolva, Abbott Vascular, and PLx Pharma; b) Participation in review 482 activities from Johnson \& Johnson, St. Jude, and Sunovion ; c) has received institutional 483 payments for grants from Bristol-Myers Squibb, Sanofi-Aventis, GlaxoSmith Kline, Otsuka, 484 Eli Lilly, Daiichi Sankyo, Inc., The Medicines Company, AstraZeneca, Evolva, Gilead; and 485 has other financial relationships with Esther and King Biomedical Research Grant. 486 Aradi D: consultancy for Verum Diagnostica $\mathrm{GmbH}$; lectures for Verum Diagnostica, Roche, 487 DSI/Lilly, Bayer, Astra-Zeneca, Pfizer, Biotronic, Abbott. 
488 Beigel R: no conflicts of interest

489 Campo G: no conflicts of interest

490 Combescure C: no conflicts of interest

7

8

9

10

11

12

13

14

15

16

17

18

19

20

21

22

23

24

25

26

27

28

29

30

31

32

33

34

35

36

37

38

39

40

41

42

43

44

45

46

47

48

49

50

51

52

53

54

55

56

57

58

59

60 


\section{References}

492 1. Eikelboom JW, Hirsh J, Spencer FA, et al. Antiplatelet drugs: Antithrombotic Therapy

493 and Prevention of Thrombosis, 9th ed: American College of Chest Physicians Evidence-

494 Based Clinical Practice Guidelines. Chest 2012; 141(2 Suppl): e89S-119S.

495 2. Yusuf S, Zhao F, Mehta SR, et al. Effects of clopidogrel in addition to aspirin in 496 patients with acute coronary syndromes without ST-segment elevation. N Engl J Med 2001; 497 345(7): 494-502.

498 3. Gurbel PA, Bliden KP, Hiatt BL, et al. Clopidogrel for coronary stenting: response 499 variability, drug resistance, and the effect of pretreatment platelet reactivity. Circulation 2003; 500 107(23): 2908-13.

501 4. Hochholzer W, Trenk D, Frundi D, et al. Time dependence of platelet inhibition after a 502 600-mg loading dose of clopidogrel in a large, unselected cohort of candidates for 503 percutaneous coronary intervention. Circulation 2005; 111(20): 2560-4.

504 5. Snoep JD, Hovens MM, Eikenboom JC, et al. Association of laboratory-defined 505 aspirin resistance with a higher risk of recurrent cardiovascular events: a systematic review 506 and meta-analysis. Arch Intern Med 2007; 167(15): 1593-9.

507 6. Aradi D, Komocsi A, Vorobcsuk A, et al. Prognostic significance of high on-clopidogrel 508 platelet reactivity after percutaneous coronary intervention: systematic review and meta509 analysis. Am Heart J 2010; 160(3): 543-51.

$5107 . \quad$ Combescure C, Fontana P, Mallouk N, et al. Clinical implications of clopidogrel non511 response in cardiovascular patients: a systematic review and meta-analysis. J Thromb 512 Haemost 2010; 8(5): 923-33.

513 8. Reny JL, Berdague P, Poncet A, et al. Antiplatelet Drug Response Status Does Not 514 Predict Recurrent Ischemic Events in Stable Cardiovascular Patients: Results of the 515 Antiplatelet Drug Resistances and Ischemic Events Study. Circulation 2012; 125(25): 320151610.

517 9. Pettersen AA, Seljeflot I, Abdelnoor M, et al. High On-Aspirin Platelet Reactivity and 518 Clinical Outcome in Patients With Stable Coronary Artery Disease: Results From ASCET 
519 (Aspirin Nonresponsiveness and Clopidogrel Endpoint Trial). Journal of the American Heart $520 \quad$ Association 2012; 1(3): e000703.

521 10. Gurbel PA, Erlinge D, Ohman EM, et al. Platelet function during extended prasugrel 522 and clopidogrel therapy for patients with ACS treated without revascularization: the 523 TRILOGY ACS platelet function substudy. JAMA 2012; 308(17): 1785-94.

524 11. Bonello L, Camoin-Jau L, Arques S, et al. Adjusted clopidogrel loading doses 525 according to vasodilator-stimulated phosphoprotein phosphorylation index decrease rate of 526 major adverse cardiovascular events in patients with clopidogrel resistance: a multicenter 527 randomized prospective study. J Am Coll Cardiol 2008; 51(14): 1404-11.

528 12. Bonello L, Camoin-Jau L, Armero S, et al. Tailored clopidogrel loading dose 529 according to platelet reactivity monitoring to prevent acute and subacute stent thrombosis. $530 \quad$ Am J Cardiol 2009; 103(1): 5-10.

531 13. Price MJ, Berger PB, Teirstein PS, et al. Standard- vs high-dose clopidogrel based on 532 platelet function testing after percutaneous coronary intervention: the GRAVITAS 533 randomized trial. JAMA 2011; 305(11): 1097-105.

534 14. Collet JP, Cuisset T, Range G, et al. Bedside monitoring to adjust antiplatelet therapy 535 for coronary stenting. N Engl J Med 2012; 367(22): 2100-9.

536 15. Aradi D, Komocsi A, Price MJ, et al. Efficacy and safety of intensified antiplatelet 537 therapy on the basis of platelet reactivity testing in patients after percutaneous coronary 538 intervention: systematic review and meta-analysis. Int J Cardiol 2013; 167(5): 2140-8.

539 16. Stone GW, Witzenbichler B, Weisz G, et al. Platelet reactivity and clinical outcomes 540 after coronary artery implantation of drug-eluting stents (ADAPT-DES): a prospective 541 multicentre registry study. Lancet 2013; 382(9892): 614-23.

542 17. Brar SS, ten Berg J, Marcucci R, et al. Impact of platelet reactivity on clinical 543 outcomes after percutaneous coronary intervention. A collaborative meta-analysis of 544 individual participant data. J Am Coll Cardiol 2011; 58(19): 1945-54. 
545 18. Yamaguchi Y, Abe T, Sato Y, et al. Effects of VerifyNow P2Y12 test and CYP2C19*2

546 testing on clinical outcomes of patients with cardiovascular disease: a systematic review and 547 meta-analysis. Platelets 2013; 24(5): 352-61.

548 19. Moher D, Pham B, Klassen TP, et al. What contributions do languages other than 549 English make on the results of meta-analyses? J Clin Epidemiol 2000; 53(9): 964-72.

550 20. Berlin JA. Does blinding of readers affect the results of meta-analyses? University of 551 Pennsylvania Meta-analysis Blinding Study Group. Lancet 1997; 350(9072): 185-6.

552 21. Bonello L, Tantry US, Marcucci R, et al. Consensus and future directions on the 553 definition of high on-treatment platelet reactivity to adenosine diphosphate. J Am Coll Cardiol $5542010 ; 56(12): 919-33$.

555 22. Tantry US, Bonello L, Aradi D, et al. Consensus and update on the definition of on556 treatment platelet reactivity to adenosine diphosphate associated with ischemia and bleeding. 557 J Am Coll Cardiol 2013; 62(24): 2261-73.

558 23. Stewart GB, Altman DG, Askie LM, et al. Statistical analysis of individual participant 559 data meta-analyses: a comparison of methods and recommendations for practice. PLoS One $560 \quad 2012 ; 7(10):$ e46042.

561 24. Akritas MG. Nearest neighbor estimation of a bivariate distribution under random 562 censoring. Ann Stat 1994; 22: 1299-327.

563 25. Pencina MJ, D'Agostino RB, Sr., Steyerberg EW. Extensions of net reclassification 564 improvement calculations to measure usefulness of new biomarkers. Stat Med 2011; 30(1): $565 \quad 11-21$.

566 26. Stroup DF, Berlin JA, Morton SC, et al. Meta-analysis of observational studies in 567 epidemiology: a proposal for reporting. Meta-analysis Of Observational Studies in 568 Epidemiology (MOOSE) group. JAMA 2000; 283(15): 2008-12.

569 27. Campo G, Valgimigli M, Gemmati D, et al. Value of Platelet Reactivity in Predicting 570 Response to Treatment and Clinical Outcome in Patients Undergoing Primary Coronary 571 Intervention. Insights Into the STRATEGY Study. Journal of the American College of 572 Cardiology 2006; 48(11): 2178-85. 
573 28. Hochholzer W, Trenk D, Bestehorn HP, et al. Impact of the degree of peri574 interventional platelet inhibition after loading with clopidogrel on early clinical outcome of 575 elective coronary stent placement. J Am Coll Cardiol 2006; 48(9): 1742-50.

576 29. Angiolillo DJ, Bernardo E, Sabate M, et al. Impact of platelet reactivity on 577 cardiovascular outcomes in patients with type 2 diabetes mellitus and coronary artery 578 disease. J Am Coll Cardiol 2007; 50(16): 1541-7.

579 30. Cuisset T, Frere C, Quilici J, et al. High post-treatment platelet reactivity is associated 580 with a high incidence of myonecrosis after stenting for non-ST elevation acute coronary 581 syndromes. Thrombosis and haemostasis $2007 ; 97(2): 282-7$.

582 31. Geisler T, Grass D, Bigalke B, et al. The Residual Platelet Aggregation after 583 Deployment of Intracoronary Stent (PREDICT) score. J Thromb Haemost 2008; 6(1): 54-61.

584 32. Gurbel PA, Antonino MJ, Bliden KP, et al. Platelet reactivity to adenosine diphosphate 585 and long-term ischemic event occurrence following percutaneous coronary intervention: a 586 potential antiplatelet therapeutic target. Platelets 2008; 19(8): 595-604.

587 33. Cuisset T, Frere C, Quilici J, et al. Predictive values of post-treatment adenosine 588 diphosphate-induced aggregation and vasodilator-stimulated phosphoprotein index for stent 589 thrombosis after acute coronary syndrome in clopidogrel-treated patients. Am J Cardiol 2009; 590 104(8): 1078-82.

591 34. Yong G, Rankin J, Ferguson L, et al. Randomized trial comparing 600- with 300-mg 592 loading dose of clopidogrel in patients with non-ST elevation acute coronary syndrome 593 undergoing percutaneous coronary intervention: results of the Platelet Responsiveness to 594 Aspirin and Clopidogrel and Troponin Increment after Coronary intervention in Acute 595 coronary Lesions (PRACTICAL) Trial. Am Heart J 2009; 157(1): 60 e1-9.

596 35. Breet NJ, van Werkum JW, Bouman HJ, et al. Comparison of platelet function tests in 597 predicting clinical outcome in patients undergoing coronary stent implantation. JAMA 2010; 598 303(8): 754-62.

599 36. Marcucci R, Gori AM, Paniccia R, et al. High on-treatment platelet reactivity by more 600 than one agonist predicts 12-month follow-up cardiovascular death and non-fatal myocardial 
601 infarction in acute coronary syndrome patients receiving coronary stenting. Thromb Haemost $602 \quad 2010 ; 104(2): 279-86$.

603 37. Beigel $\mathrm{R}$, Hod $\mathrm{H}$, Fefer $\mathrm{P}$, et al. Relation of aspirin failure to clinical outcome and to 604 platelet response to aspirin in patients with acute myocardial infarction. Am J Cardiol 2011; 605 107(3): 339-42.

606 38. Aradi D, Rideg O, Vorobcsuk A, et al. Justification of $150 \mathrm{mg}$ clopidogrel in patients 607 with high on-clopidogrel platelet reactivity. Eur J Clin Invest 2012; 42(4): 384-92.

608 39. Gagne JJ, Bykov K, Choudhry NK, et al. Effect of smoking on comparative efficacy of 609 antiplatelet agents: systematic review, meta-analysis, and indirect comparison. BMJ 2013; $610 \quad 347:$ f5307.

611 40. Lafitte M, Pucheu Y, Latry K, et al. Predictors of cardiovascular prognosis in patients 612 receiving optimized secondary prevention measures after acute coronary syndrome. 613 European journal of preventive cardiology 2013; 20(2): 283-90.

614 41. Cattaneo M. Aspirin and clopidogrel: efficacy, safety, and the issue of drug resistance. 615 Arterioscler Thromb Vasc Biol 2004; 24(11): 1980-7.

616 42. Jeong YH, Bliden KP, Antonino MJ, et al. Usefulness of the VerifyNow P2Y12 assay 617 to evaluate the antiplatelet effects of ticagrelor and clopidogrel therapies. Am Heart J 2012; 618 164(1): 35-42.

619 43. Van Werkum J, Van Der Stelt CA, Seesing TH, et al. A head-to-head comparison 620 between the VerifyNow P2Y12 assay and light transmittance aggregometry for monitoring 621 the individual platelet response to clopidogrel in patients undergoing elective percutaneous 622 coronary intervention. J Thromb Haemost 2006; 4(11): 2516-8.

623 44. Coleman $\mathrm{Cl}$, Limone BL. Universal versus platelet reactivity assay-driven use of 624 P2Y12 inhibitors in acute coronary syndrome patients: cost-effectiveness analyses for six 625 European perspectives. Thromb Haemost 2014; 111(1): 103-10.

626 45. Cayla G, Cuisset T, Silvain J, et al. Platelet function monitoring in elderly patients on 627 prasugrel after stenting for an acute coronary syndrome: Design of the randomized antarctic 628 study. American heart journal 2014; 168(5): 674-81. 
629

630

5

6

11

12

13

14

15

16

17

18

19

20

21

22

23

24

25

26

27

28

29

30

31

32

33

34

35

36

37

38

39

40

41

42

43

44

45

46

47

48

49

50

51

52

53

54

55

56

57

58

59

60 
Table 1. Main characteristics of published studies

\begin{tabular}{|c|c|c|c|c|c|c|c|c|c|c|c|c|c|}
\hline Study & $\begin{array}{c}\text { Years of } \\
\text { publication }\end{array}$ & $\begin{array}{c}\text { Patients } \\
\text { (n) }\end{array}$ & $\begin{array}{l}\text { Age } \\
(y)\end{array}$ & $\begin{array}{c}\text { Male } \\
(\%)\end{array}$ & $\begin{array}{c}\text { Diabetics } \\
(\%)\end{array}$ & $\begin{array}{c}\text { Smokers } \\
(\%)\end{array}$ & $\begin{array}{l}\text { Hyper- } \\
\text { tension } \\
(\%)\end{array}$ & $\begin{array}{c}\text { Hypercholes- } \\
\text { terolemia } \\
(\%)\end{array}$ & $\begin{array}{c}\text { ACS at } \\
\text { inclusion } \\
(\%)\end{array}$ & $\begin{array}{l}\mathrm{PCl} \\
(\%)\end{array}$ & $\begin{array}{c}\text { Gpllb/llla } \\
\text { inhibitor } \\
(\%)\end{array}$ & $\begin{array}{l}\text { Follow-up } \\
\text { (months) }^{*}\end{array}$ & $\begin{array}{l}\text { ADP } \\
(\mu \mathrm{M})\end{array}$ \\
\hline Campo et al.(27) & 2006 & 70 & $64 \pm 13$ & 69 & 19 & 37 & 63 & 34 & 100 & 100 & 100 & $10(15)$ & 5,20 \\
\hline Hochholzer et al.(28) & 2006 & 765 & $66 \pm 9$ & 78 & 24 & 11 & 82 & 92 & 0 & 100 & 0 & $12(12)$ & 5,20 \\
\hline Angiolillo et al.(29) & 2007 & 173 & $67 \pm 9$ & 65 & 100 & 13 & 65 & 68 & 0 & 0 & 0 & $24(36)$ & 20 \\
\hline Cuisset et al.(30) & 2007 & 190 & $65 \pm 12$ & 76 & 33 & 48 & 58 & 53 & 87.4 & 100 & 14.7 & $1(1)$ & 10,20 \\
\hline Geisler et al.(31) & 2008 & 1,092 & $67 \pm 11$ & 74 & 33 & 39 & 80 & 59 & 51.7 & 100 & 7.7 & $1(1)$ & 20 \\
\hline Gurbel et al.(32) & 2008 & 297 & $65 \pm 12$ & 65 & 41 & 55 & 74 & 82 & 0 & 100 & 42 & $24(24)$ & 5,20 \\
\hline Cuisset et al.(33) & 2009 & 598 & $65 \pm 12$ & 78 & 35 & 39 & 56 & 55 & 100 & 100 & 9.9 & $1(1)$ & 10 \\
\hline Yong et al.(34) & 2009 & 248 & $63 \pm 12$ & 71 & 22 & 27 & 53 & 52 & 100 & 55 & 39.7 & $6(21)$ & $5,10,20$ \\
\hline Breet et al.(35) & 2010 & 1,069 & $64 \pm 11$ & 75 & 81 & 11 & 77 & 80 & 0 & 100 & 7.0 & $12(12)$ & 5,20 \\
\hline Marcucci et al.(36) & 2010 & 1,108 & $69 \pm 10$ & 75 & 24 & 23 & 66 & 55 & 100 & 100 & 26.0 & $12(12)$ & 10 \\
\hline Beigel et al.(37) & 2011 & 174 & $59 \pm 12$ & 83 & 27 & 41 & 51 & 45 & 100 & 100 & - & $6(6)$ & 5 \\
\hline Aradi et al.(38) & 2012 & 160 & $62 \pm 9$ & 63 & 38 & 36 & 84 & 50 & 0 & 100 & 0 & $12(12)$ & 5 \\
\hline Reny et al.(8) & 2012 & 534 & $62 \pm 12$ & 82 & 21 & 20 & 56 & 63 & 0 & 0 & 0 & $32(50)$ & 5,20 \\
\hline
\end{tabular}

633 Age, mean \pm standard deviation; CAD, coronary artery disease; ACS, acute coronary syndrome; PCl, percutaneous coronary intervention; ADP,

634 adenosine diphospate concentration used for the evaluation of platelet reactivity

$635{ }^{*}$ Median (maximum) 
637 Table 2: Multivariate analysis to assess the associations between the risk factors and the composite outcome of MACE. This analysis was 638 conducted on the patients of the 13 studies of the meta-analysis ( $n=6,256$ after exclusion of missing data). MACE were observed in 412 patients.

639 Hazard ratios (HR) greater than one show an increased risk of MACE in patients having the corresponding risk factor.

\begin{tabular}{|l|c|c|l|c|c|}
\hline Factors collected in studies & Adjusted HR [95\% Cl] & $\mathbf{p}$ & Level of risk of MACE * & HR [95\% Cl] & $\mathbf{p}$ \\
\hline Current smoking status & $0.92[0.71 ; 1.18]$ & 0.50 & Low risk $(n=579)$ & 1 & \\
\hline Age $>75)$ & $1.56[1.25 ; 1.95]$ & $<0.0001$ & Intermediate risk $(n=2444)$ & $1.61[1.05 ; 2.45]$ & 0.03 \\
\hline Diabetes & $1.58[1.27 ; 1.96]$ & $<0.0001$ & High risk $(n=3435)$ & $2.58[1.69 ; 3.94]$ & $<0.0001$ \\
\hline Hypercholesterolemia & $0.86[0.69 ; 1.06]$ & 0.15 & & & \\
\hline Hypertension & $1.23[0.98 ; 1.54]$ & 0.07 & & & \\
\hline ACS at inclusion & $2.00[1.27 ; 3.16]$ & 0.003 & & & \\
\hline Gender (Male) & $1.11[0.89 ; 1.40]$ & 0.35 & & & \\
\hline
\end{tabular}

: a surrogate for the level of risk was defined as the number of risk factors (among age, diabetes, hypertension, and ACS at inclusion): low risk for 641 no risk factor, intermediate risk for one risk factor and high risk for two or more risk factors). 
644 Table 3: Associations between the ADP induced-aggregation categories and the composite outcome of MACE with adjustment on the factors 645 collected in the studies of the meta-analysis (factors shown in Table 2).

\begin{tabular}{|c|c|c|c|c|c|c|}
\hline & \multicolumn{2}{|c|}{ ADP $20 \mu \mathrm{M}$} & \multicolumn{2}{|c|}{ ADP $10 \mu \mathrm{M}$} & \multicolumn{2}{|c|}{ ADP $5 \mu \mathrm{M}$} \\
\hline & $\mathbf{N}$ & & $\mathbf{N}$ & & $\mathbf{N}$ & \\
\hline Studies & 9 & & 4 & & 8 & \\
\hline Events & 287 & & 124 & & 229 & \\
\hline Patients (after exclusion of missing data) & 4,140 & & 2,077 & & 3,160 & \\
\hline & HR $[95 \% \mathrm{Cl}]$ & p & HR [95\% Cl] & $p$ & HR [95\% Cl] & $p$ \\
\hline ADP induced-aggregation categories & & $0.0003 * *$ & & $0.03 * *$ & & $0.02^{* *}$ \\
\hline Lower category * & 1 & & 1 & & 1 & \\
\hline Intermediate category ${ }^{*}$ & $1.85[1.26 ; 2.73]$ & 0.002 & $1.31[0.79 ; 2.17]$ & 0.30 & $1.79[1.02 ; 3.14]$ & 0.04 \\
\hline Higher category* & $2.91[1.78 ; 4.74]$ & $<0.0001$ & $2.61[1.64 ; 4.16]$ & $<0.0001$ & $2.79[1.50 ; 5.22]$ & 0.001 \\
\hline
\end{tabular}

HR, Hazard Ratio; $\mathrm{Cl}$, Confidence Interval

$647 *$ Categories for ADP 20 and $10 \mu \mathrm{M}$ are 0\%-40\%, 41\%-60\%, 61\%-100\%, and for ADP $5 \mu \mathrm{M}$ are 0\%-30\%, 31\%-50\%, 51\%-100\%

$648 * *$ : global $\mathrm{p}$-values for testing the hypothesis that both HRs (intermediate- and higher-ADP induced-aggregation category) equal 1 
650 Table 4: Reclassification of the 6-month risk of MACE when the individual risk was predicted from platelet reactivity measured by $20 \mu \mathrm{M}$ ADP in 651 addition to risk factors. The predicted risk was stratified in three levels (low: $\leq 3 \%$, intermediate: $>3 \%$ and $\leq 5 \%$, high: $>5 \%$ ) in agreement with the 6 652 month risk observed in patients with none, one and two or more risk factors $(2.3 \%, 4.1 \%$ and $6.2 \%$ respectively). Patients were stratified according 653 to their number of risk factors and to the level of the predicted risk. The numbers of patients and, in brackets, the corresponding observed 6-month 654 risk of MACE in each stratum.

\begin{tabular}{|l|c|c|c|c|}
\hline & \multicolumn{4}{|c|}{ Risk predicted by the combination of risk factors and platelet reactivity measured by } \\
$20 \mu \mathrm{M}$ ADP
\end{tabular}

*: number of patients

$656{ }^{* *}$ : observed 6-month risk of MACE 
659 Figure 1: Flow chart of the meta-analysis

660

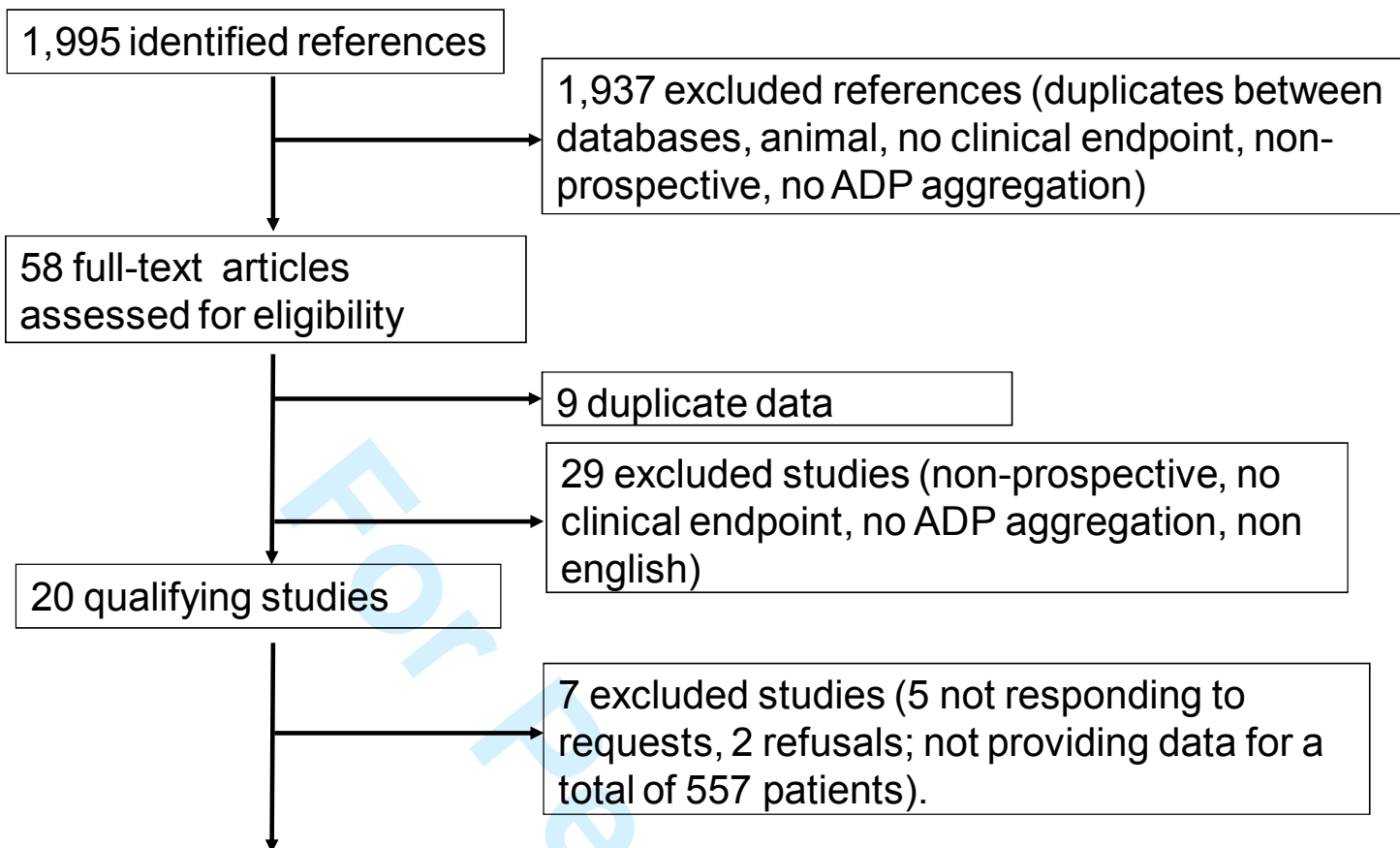

13 included studies

661 totaling 6,478 patients 
662 Figure 2: Kaplan-Meier survival curve for the occurrence of MACE

663

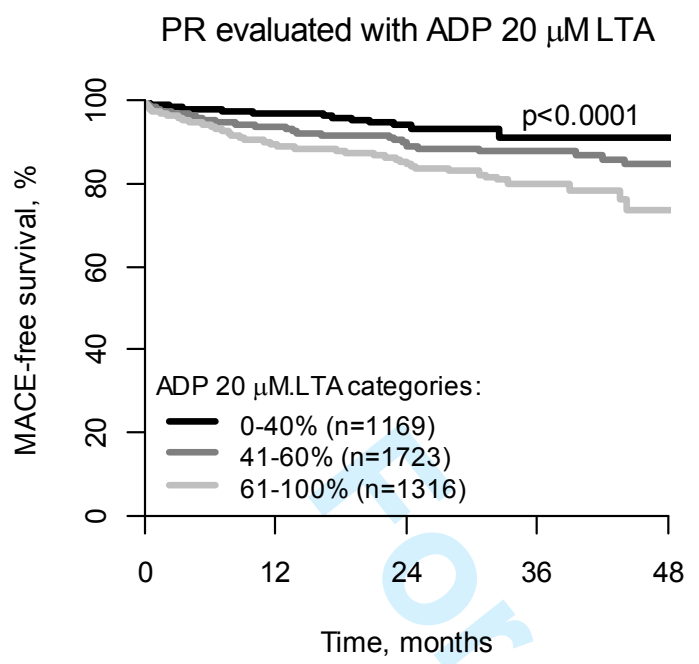

PR evaluated with ADP $10 \mu \mathrm{M} \mathrm{LTA}$
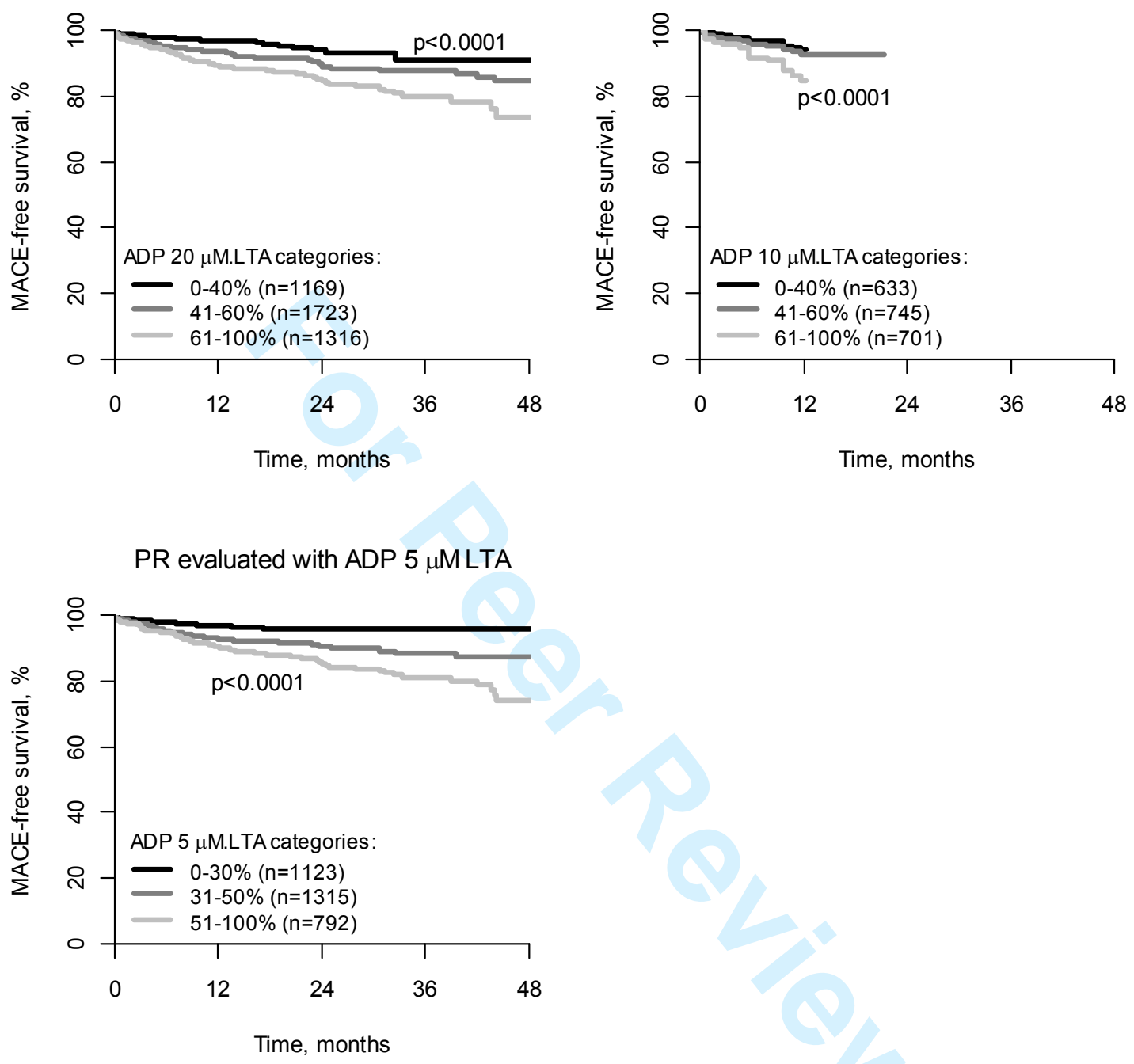

664 
665 Figure 3: Association between platelet reactivity and the occurrence of MACE according to 666 the level of risk. Low-risk patients have none of the risk factors (among age $>75$ years, acute 667 coronary syndrome at inclusion, diabetes, and hypertension), intermediate-risk patients have 668 one risk factor and high-risk patients have two or more risk factors. PR was assessed with 20 $669 \mu \mathrm{M}$ ADP LTA.

670
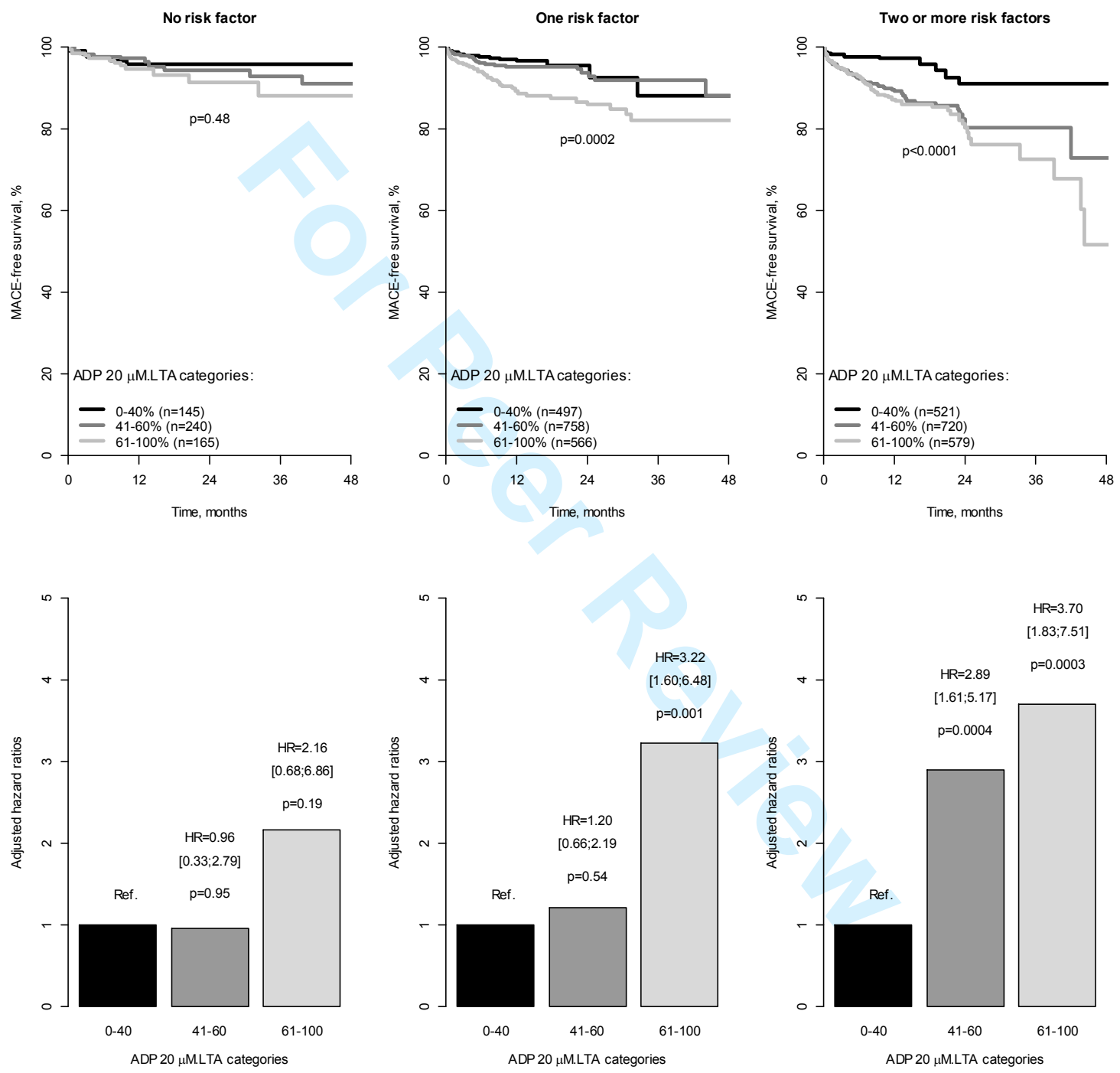

671

49

50

51

52

53

54

55

56 
Figure 4: 6-month risk of MACE according to platelet reactivity in the different risk groups. The dashed line represents the overall risk, ignoring platelet reactivity and the black line shows the risk according to the platelet reactivity assessed with $20 \mu M$ ADP LTA, in patients with no risk factors $(A)$, one risk factor $(B)$ and two or more risk factors $(C)$.
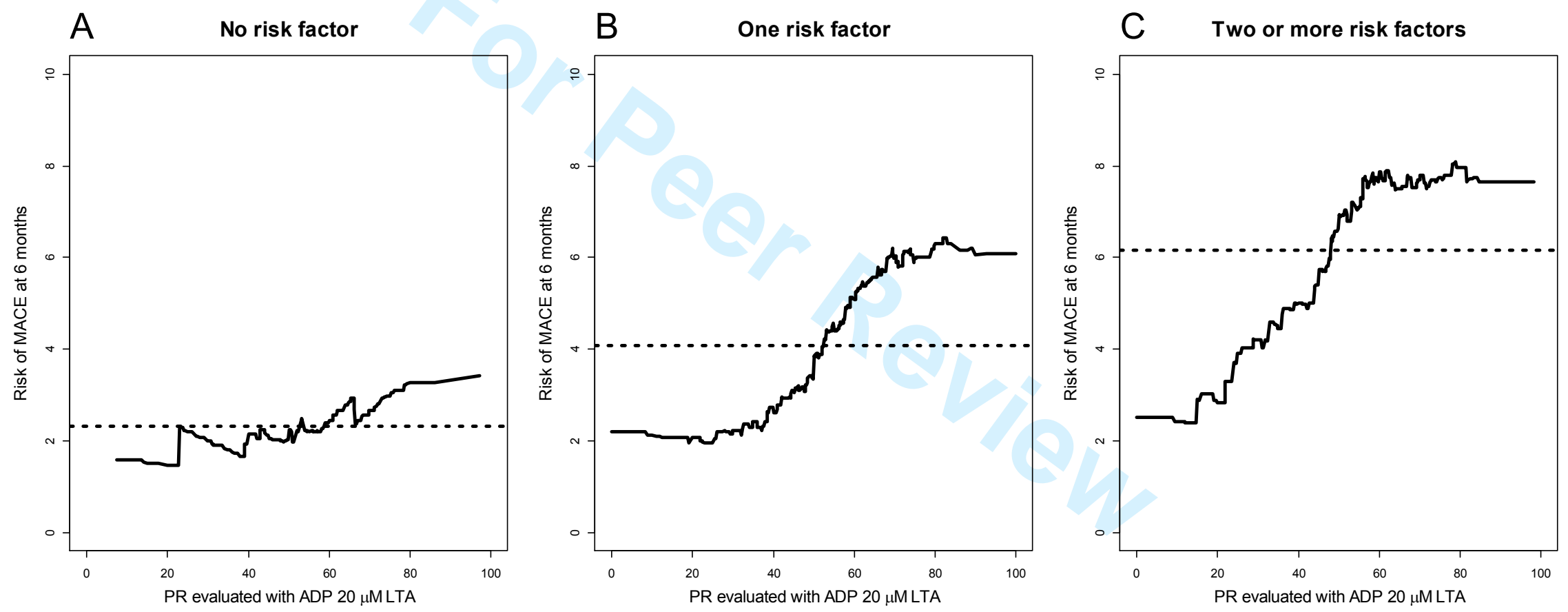
1 Title: Vascular risk levels affect the predictive value of platelet reactivity for the occurrence of

2 major adverse cardiovascular events in patients on clopidogrel: Systematic review and

3 collaborative meta-analysis of individual patient data

4

Running head: Vascular risk, platelet reactivity, and prognosis

\section{Authors:}

Jean-Luc Reny; Pierre Fontana; Willibald Hochholzer; Franz Josef Neumann; Jurriën ten Berg; Paul W.Janssen; Tobias Geisler; Meinrad Gawaz; Rossella Marcucci; Anna-Maria Gori; Thomas Cuisset; Marie-Christine Alessi; Philippe Berdagué; Paul A. Gurbel; Gerald Yong; Dominick J. Angiolillo; Daniel Aradi; Roy Beigel; Gianluca Campo; and Christophe Combescure.

\section{Authors' affiliations:}

Geneva Platelet Group, School of Medicine, University of Geneva, Geneva, Switzerland (Dr. Reny, Dr. Fontana); Division of Internal Medicine and Rehabilitation, Trois-Chêne Hospital, Geneva University Hospitals, Geneva, Switzerland (Dr. Reny); Division of Angiology and Haemostasis, Geneva University Hospitals, Geneva, Switzerland (Dr. Fontana); Universitaets Herzzentrum Freiburg-Bad Krozingen, Klinik für Kardiologie und Angiologie II, Bad Krozingen, Germany (Dr. Hochholzer, Dr. Neumann); St Antonius Hospital, Nieuwegein, the Netherlands (Dr. ten Berg, Dr. Janssen); University Hospital Tübingen, Department of cardiology, Tübingen, Germany (Dr. Geisler, Dr. Gawaz) Center for atherothrombotic diseases, Dept Experimental and Clinical Medicine, University of Florence, Azienda Ospedaliero Universitaria Careggi, Florence, Italy (Dr. Marcucci, Ms Gori); Cardiologie Interventionnelle, CHU Timone Unité Inserm UMR 626, Faculté de Médecine Marseille, France (Dr Cuisset); UMR Inserm 1062, inra 1260, université Aix-Marseille, France (Dr Alessi); Division of Cardiology, Beziers Hospital, Béziers, France (Dr Berdagué); Sinai Center for Thrombosis Research, Sinai Hospital of Baltimore, Baltimore, USA (Dr Gurbel); Maryland Royal Perth Hospital, Australia (Dr. Yong); Division of Cardiology, University of Florida College of Medicine, Jacksonville, Florida, USA (Dr. Angiolillo); Department of Cardiology, Heart Center Balatonfüred, Hungary (Dr. Aradi); The Leviev Heart Center, Sheba Medical Center, Tel-Hashomer, and the Sackler Faculty of Medicine, Tel Aviv University, TelAviv, Israel (Dr. Beigel); Cardiovascular Institute, Azienda Ospedaliera Universitaria di Ferrara, Cona (FE), Italy (Dr Campo); Division of Clinical Epidemiology, University Hospitals of Geneva, Geneva, Switzerland (Dr. Combescure), Center of Clinical Research, Faculty of Medicine, University of Geneva, Geneva, Switzerland (Dr. Combescure)

\section{Corresponding author:}

Jean-Luc Reny

Division of Internal Medicine and Rehabilitation

Trois-Chêne Hospital, Geneva University Hospitals

Ch. du Pont-Bochet 3

$\mathrm{CH}-1226$ Thônex-Geneva

Switzerland

Phone: +41-22-305 6537

Fax: +41-22-305 6115

E-mail: jean-luc.reny@hcuge.ch 
Abstract

Prior studies have shown an association between high on-clopidogrel platelet reactivity (PR) and the risk of major adverse cardiovascular events (MACE). However, large intervention trials on PR-tailored treatments have been neutral. The role and usefulness of PR with regard to levels of cardiovascular risk are unclear. We undertook a systematic review and meta-analysis of individual patient data on MACE outcomes (acute coronary syndromes (ACS), ischemic strokes, and vascular deaths) in relation to PR and its interaction with cardiovascular risk levels. PR was determined using ADP-induced light transmission aggregometry with a primary concentration of $20 \mu \mathrm{M}$ ADP. Thirteen prospective studies totaled 6,478 clopidogrel-treated patients who experienced 421 MACE (6.5\%) during a median follow-up of 12 months. The strength of the association between the risk of MACE and PR increased significantly $(p=0.04)$ with the number of risk factors present (age $>75$ years, ACS at inclusion, diabetes, and hypertension). No association was detected in patients with no risk factor $(p=0.48)$. In patients presenting one risk factor, only high-PR was associated with an increased risk of MACE (HR 3.2, $p=0.001)$. In patients presenting $\geq 2$ risk factors, the increase of risk started from medium-PR (medium-PR: HR=2.9, $p=0.0004$; highPR: $H R=3.7, p=0.0003)$. PR allowed the reclassification of $44 \%$ of the total population to a different risk level for the outcome of MACE, mostly in intermediate or high risk patients. In conclusion, the magnitude of the association between PR and MACE risk is strongly dependent on the level of cardiovascular risk faced by patients on clopidogrel.

Keywords: clopidogrel, drug response, platelets, cardiovascular diseases, ischemic events. 


\section{Introduction}

Atherosclerotic diseases account for more than $40 \%$ of deaths in Western countries, and antiplatelet therapy is a major preventive strategy in this setting(1). Clopidogrel, a P2Y 12 receptor blocker, inhibits the activation of platelets by adenosine diphosphate (ADP), and is widely prescribed for secondary prevention in patients with atherosclerotic diseases. When combined with aspirin, clopidogrel is particularly effective in patients with acute coronary syndromes (ACS)(2), and has proved superior to aspirin alone in several other large randomised controlled trials. The pharmacodynamic response to clopidogrel shows a wide inter-individual variability $(3,4)$. Numerous cohort studies, often performed on patients with acute coronary syndrome (ACS) and/or undergoing percutaneous coronary interventions $(\mathrm{PCl})$, have shown an association between high on-treatment platelet reactivity $(\mathrm{PR})$ and the risk of recurrent major adverse cardiovascular events (MACE)(5-7). However, recent studies in cohorts of stable cardiovascular outpatients $(8,9)$ or in medically managed ACS patients(10) failed to confirm these results. Several randomized trials aimed at reducing the recurrence of ischemic events have compared standard clopidogrel treatment to a $\mathrm{P}_{2} \mathrm{Y}_{12^{-}}$ inhibitor strategy tailored according to the presence of high PR. Although initial small trials were promising $(11,12)$ more recent larger trials showed no benefit from adjusting clopidogrel doses or switching to prasugrel based on PR testing in low-risk coronary patients undergoing $\mathrm{PCI}(13,14)$. These contrasting results, both from observational studies and randomized intervention trials, may be explained by different patient characteristics including the level of risk, but to date few data substantiate these hypotheses. We previously showed, in a studylevel meta-analysis, that the risk of recurrent MACE associated with high PR was greater in studies using Gpllb/llla inhibitors (a marker of high-risk patients) than in studies which did not(7). Another meta-regression from a study-level meta-analysis of randomized trials suggested that the higher the incidence of coronary stent thrombosis in a given study, the larger the net clinical benefit from a PR-tailored strategy(15). Finally, the ADAPT-DES registry of patients undergoing $\mathrm{PCl}$ showed that high $\mathrm{PR}$ was predictive of stent thrombosis mostly in ACS patients, but there was no interaction reported between PR and the presence 
101 of an ACS at inclusion(16). This information suggests the hypothesis that high PR might be

102 more relevant in high-risk populations, but convincing data at the individual level are lacking.

103 To date, the only meta-analysis on individual patient data performed on 6 studies totaling

1043,059 patients assessed with the VerifyNow P2Y12 assay did not explore this hypothesis(17).

105 Similarly, one of the largest and more recent meta-analysis on 8 studies and 4817 patients

106 did not explore this interaction due to the lack of individual data(18). To further investigate

107 this interaction on a larger population we performed a collaborative meta-analysis of

108 individual patient data and focused on the interaction between relevant vascular risk factors

109 and PR, assessed with ADP induced light transmission aggregometry (LTA), in order to

110 better define the risk of MACE. ADP-induced LTA is the assay upon which all P2Y ${ }_{12}$ receptor

111 inhibitors have been developed, thus supporting its use in the present meta-analysis. In

112 addition, among several available assays to evaluate PR, LTA is the historical gold standard

113 with which most platelet function assays were compared. 


\section{Data sources}

117 Literature review, confined to articles in English(19), was based on electronic databases 118 (Medline, Embase, Web of Science, Cochrane Central Register of Controlled Trials) and 119 abstracts from major international meetings held from 2010-2013 (ISTH, AHA, ACC, ESC). 120 A free-text search was conducted using an 'ADP' and 'aggregation' and 'clopidogrel' key121 word combination. Articles were selected on the basis of abstracts, before examination of the 122 full text. Reference lists of selected articles were also hand-searched to identify additional 123 relevant reports. Reviewers (JLR and PF) were not blinded to the journal, authors or 124 institutions in the publications as this has been shown to be unnecessary(20). The electronic 125 database search was last updated on 31 July, 2013. The objective of this individual patients' 126 data meta-analysis was described in a project that was part of French ministry of health's 127 initiative to encourage meta-analyses (PHRC 15-07 to JL Reny "Etudes prospectives sur la 128 réponse biologique au clopidogrel et évènements ischémiques chez les patients 129 athérothrombotiques : Métaanalyse sur données individuelles et résumées" http://www.plan130 alzheimer.gouv.fr/IMG/pdf/Liste des dossiers retenus - 2 mai 2008.pdf ). Protocol in 131 French available upon request.

134 Selected studies met the following criteria: (a) patients were treated with clopidogrel and had 135 symptomatic atherothrombosis (clinical signs related to vascular atherothrombotic lesions); 136 (b) pharmacodynamic response to clopidogrel was evaluated using the maximal aggregation 137 value from LTA on platelet-rich plasma with 20, 10, or $5 \mu$ M ADP as an agonist; (c) LTA was 138 performed remote from platelet function interfering drugs such as Gpllb/llla inhibitors; (d) 139 patients were prospectively monitored for MACE for at least 30 days, defined using at least 140 one of the following items: acute coronary syndrome (unstable angina, myocardial infarction 141 with/without ST segment elevation), ischemic stroke (acute neurological deficit due to a 142 cerebral infarction), and vascular death; (e) studies involved either a prospective cohort or a 
randomised therapeutic trial, but one in which treatment was allocated independently of the response to clopidogrel. When studies were suspected of including the same patients, the authors were asked to provide data from the largest possible number of independent patients.

Data extraction

148 The corresponding authors or principal investigators of eligible studies were contacted and 149 asked to participate in the CLOpidogrel and Vascular ISchemic events - Individual Patient Data (CLOVIS-IPD) meta-analysis group. Investigators provided individual data on: the 151 qualifying cardiovascular condition and clinical setting at inclusion (ACS or stable disease); 152 MACE and date of occurrence during follow-up; platelet reactivity (PR) with ADP 20, 10, 153 and/or $5 \mu \mathrm{M}$ and its timing relative to loading dose of clopidogrel; age, gender, height, and 154 weight; current smoking status, diabetes, hypercholesterolemia, and hypertension; left 155 ventricular ejection fraction; platelet count; PCl; use of Gpllb/llla inhibitors and timing; 156 concomitant medications; and bleeding events and timing during follow-up. Data were 157 checked for completeness and consistency with published reports. Any discrepancies were 158 resolved with the corresponding authors. After format harmonization, data were compiled for 159 statistical analysis. All studies were approved by their respective institutional review boards. 160

161 Quality assessment of studies

162 A new quality assessment tool for prognostic studies called PROBAST (see

163 Acknowledgements) was used to estimate risks of bias and concerns about applicability. As 164 PROBAST is not customized for meta-analyses of individual patient data, items were 165 adapted accordingly. Based on the present study's list of relevant criteria, risks of bias, and 166 concerns about applicability are rated as low, unclear, or high. Supplemental Figure 1 shows 167 the list of criteria. 
170 The primary clinical outcome was the occurrence of MACE, as defined above (see Study 171 selection (d)). The primary biological outcome was maximal aggregation with $20 \mu \mathrm{M}$ ADP, as 172 it is a better concentration for analyzing the effects of clopidogrel than lower ones. PR was 173 categorized in three strata. The higher cut-offs were selected on the basis of previously 174 published cut-offs (59\% to $64 \%$ for $20 \mu \mathrm{M} \mathrm{ADP}$, and $43 \%$ to $46 \%$ for $5 \mu \mathrm{M} \mathrm{ADP)(21),} \mathrm{and} \mathrm{to}$ keep relatively balanced numbers of patients in each PR categories. Three pre-specified categories allowed a better description of the dose-dependent effects of PR on the risk of MACE compared to the usual dichotomous high and low PR categorization. Three categories were also chosen to better parallel the analysis with a therapeutic PR window that has been associated with optimal net clinical benefit(22). A surrogate for the level of cardiovascular risk was defined as the number of factors with homogeneous definitions across studies, and these were markers of MACE in the meta-analysis. The factors were selected from among age, diabetes, hypertension, smoking, hypercholesterolemia, and the presence of an ACS at inclusion (as defined in study selection (d)), and were all provided at the time of inclusion and PR testing.

\section{Statistical analysis}

MACE-free survival curves were derived from individual patient data using the Kaplan-Meier estimator; curves were compared using log-rank tests stratified by study. Associations between conventional risk factors, PR strata, and risk of MACE were analyzed using multivariate, mixed-effect Cox models. The amount of heterogeneity was assessed by the size of the random effects $\left(\mathrm{Tau}^{2}\right)$ which is an estimate of the between study variability(23). The presence of heterogeneity was tested by comparing models with and without random effects (likelihood ratio test). The interactions between the level of risk and PR strata were tested. MACE-free survival according to PR, as a continuous variable, was assessed using the R package prodlim using the symmetrical nearest neighborhoods method.(24) Sensitivity analyses were conducted to check the robustness of the findings with respect to: the risks of bias and concerns about the applicability of studies; the definition of MACE, including target 
vessel revascularization or $\mathrm{PCl}$ at inclusion, and; the influence of a given specific study. The net reclassification index (NRI) for survival data(25) was computed to quantify the contribution of PR testing for the prediction of the 6-month risk of MACE in patients with increasing numbers of traditional risk factors. The event and non-event continuous NRIs were reported. Potential publication bias was checked for. P-values below 0.05 were considered significant and all tests were two-sided. Published guidelines for meta-analysis of observational studies in epidemiology (MOOSE) and their reporting(26) were followed. Details on statistical methods are given in the online data supplement.

\section{Results}

\section{Characteristics of included studies}

The Figure 1 flow-chart details how 13 of 20 qualifying studies were included, totaling 6,478 patients(8, 27-38). Table 1 shows their characteristics. Data on body mass index, concomitant medications, left ventricular ejection fraction, or the occurrence of target and non-target vessel revascularization during follow-up were only available in some studies. All studies provided individual data allowing a homogeneous definition of MACE, current smoking status, ACS, diabetes (fasting plasma glucose $\geq 7.0 \mathrm{mmol} / \mathrm{l}$, 2-h plasma glucose $\geq$ $11.1 \mathrm{mmol} / \mathrm{l}$ after $75 \mathrm{~g}$ oral glucose load or background therapy for diabetes), and hypertension (systolic blood pressure $\geq 140 \mathrm{mmHg}$ or diastolic blood pressure $\geq 90 \mathrm{mmHg}$ or a documented history of hypertension). Hypercholesterolemia was not defined in a homogeneous fashion across studies and plasma LDL-cholesterol levels were not available for more than 2,000 patients. Overall, risks of bias and concerns about applicability were low (online data supplement further details study characteristics, bias, and applicability). Information on bleeding was limited to five studies, with only 67 major and 20 moderate/minor bleedings.

MACE and level of risk 
Overall, 421 MACE occurred in 6,478 patients $(6.5 \%)$, the majority being ACS $(n=383)$. There were 83 stent thromboses, including 79 definite or probable and four possible ones, all included in the composite outcome of MACE. The MACE-free survival rate across the different studies at the end of follow-up ranged from $77.4 \%$ to $97.3 \%$. In a multivariate analysis, four factors were found relevant to determining patients' levels of risk: age greater than 75 years, diabetes, ACS at inclusion, and hypertension (Table 2). The number of these factors was used as a surrogate for the individual risk of MACE. Patients with none of these factors were classified 'low-risk', patients with one factor 'intermediate-risk', and patients with two or more factors 'high-risk' (global p-value $<0.0001$ for the trend).

\section{MACE and PR}

Nine studies ( $n=4,438$ patients) performed LTA using $20 \mu$ M ADP, four studies $(n=2,144$ patients) used $10 \mu \mathrm{M}$ ADP, and eight studies ( $n=3,317$ patients) used $5 \mu \mathrm{M}$ ADP. Figure 2 shows the MACE-free survival curves by category of ADP concentration. Risk of MACE increased significantly with PR with $20 \mu \mathrm{M}$ ADP, $10 \mu \mathrm{M}$ ADP, and $5 \mu \mathrm{M}$ ADP. With adjustment, high PR was still significantly associated with an increased risk of MACE (Table 3). However, for PR evaluated using $10 \mu \mathrm{M}$ ADP, risk only increased for the highest PR category, corresponding to LTA values greater than $60 \%$.

Interaction between risk level and PR for the outcome of MACE

Platelet reactivity assessed with $20 \mu \mathrm{M}$ ADP. Patients with none of the four risk factors showed no significantly increased risk associated with $\mathrm{PR}$, while for patients with one risk factor only, the higher strata of PR was associated with an increased risk of MACE. Patients with two or more risk factors showed an increased risk of MACE for both the medium and higher strata of PR. (Figure 3). In a Cox model, the interaction between PR strata and the risk level was statistically significant $(p=0.04)$. The corresponding hazard ratios $(H R s)$ are shown in Figure 3. Heterogeneity was not detected for the overall interaction $(p=0.81)$, as 
well as when it was restricted to each risk level category (intermediate versus low risk level, $p=0.45$, and high versus low risk level, $p=0.90$ ). Additional results on heterogeneity are provided in the supplemental material. Figure 4A shows that PR, when analyzed in a continuous fashion, barely affects the risk of MACE at 6 months in patients with no risk factors: the risk is close to $2 \%$ at six months, irrespective of the level of platelet reactivity. Conversely, patients with one risk factor and an overall $4.1 \%$ risk of MACE at six months have in fact a $2 \%$ risk of MACE when they have a low PR, or a $6 \%$ risk of MACE when they have a high PR (Figure 4B). Similarly, patients with two or more risk factors and an overall $6 \%$ risk of MACE at six months can indeed have a $2 \%$ risk of MACE when they have a low PR (Figure 4C). The reclassification of the 6-month risk of MACE, according to the three categories of platelet reactivity, in patients with no, one and two or more risk factors, is shown in Table 4. Overall, PR allowed the reclassification of $44 \%$ of the total population (1837/4193 patients) included in a 6-month follow-up to a different level, mostly in patients originally identified as intermediate or high risk on the basis of the number of risk factors only. In patients experiencing MACE in the first 6 months of follow-up, the risk predicted by the combination of $\mathrm{PR}$ and risk factors was on average increased compared with the risk predicted from risk factors only: the continuous event net reclassification index (NRI) was $0.39(95 \% \mathrm{Cl} 0.23$ to 0.62$)$. Conversely, in patients free of MACE at 6 months, the measure of 271 PR did not modify the predicted risk: the continuous non-event NRI was $0.01(95 \% \mathrm{Cl}-0.16$ to 272 0.09). The overall NRI was $0.39(95 \% \mathrm{Cl} 0.22$ to 0.57$)$. studies performing $10 \mu \mathrm{M}$ ADP LTA to assess PR precluded an analysis of this low-risk 276 group. Furthermore, the surrogate for risk level failed to demonstrate an association with the observed risk of MACE in these studies. Figure 4B shows that the risk of MACE increased in both intermediate- and high-risk patients for PR values above $40 \%$, without any obvious relation with the level of risk. 
281 Platelet reactivity assessed with $5 \mu \mathrm{M}$ ADP. The direction of interaction between PR using 5

$282 \mu \mathrm{M}$ ADP and the risk level was similar to that observed for PR using $20 \mu \mathrm{M}$ ADP, even

283 though overall interaction did not reach the significance level $(p=0.17)$. Of note there were

284980 fewer patients in the studies performing $5 \mu \mathrm{M}$ ADP than in those using $20 \mu \mathrm{M}$ ADP. The

285 increased risk of MACE as PR increases is indeed similar for intermediate- and high-risk

286 patients; for low-risk patients PR is not associated with a MACE outcome (online data

287 supplement). Heterogeneity was not detected for the overall interaction $(p=0.19)$. Figure $4 C$

288 shows that the risk of MACE was unaffected by PR in low-risk patients while it increased for 289 PR values above $30 \%$ in intermediate-risk patients and for PR values above $10 \%-20 \%$ in 290 high-risk patients.

\section{Sensitivity analyses}

Sensitivity analyses were performed for PR using $20 \mu \mathrm{M}$ ADP to assess: the robustness of the association between PR and risk of MACE and its interaction with the level of cardiovascular risk; the robustness of the results in the population of $\mathrm{PCl}$ patients and when target vessel revascularization is added to the composite outcome. All analyses showed that the sizes of the effects remained similar, and whilst in some instances the statistical significance of the interactions could be lost, there was no impact on their magnitudes

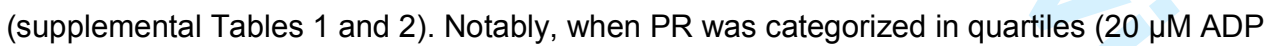
maximal aggregation quartiles $=0 \%-38.1 \%, 38.2 \%-51.3 \% .51 .4 \%-63.0 \%, 63.1 \%-100 \%$ ) the interaction between PR and the number of risk factors remained significant $(p=0.01)$. When restricted to the population of 3,564 patients treated with $\mathrm{PCl}$ and tested using $20 \mu \mathrm{M}$ ADP the interaction was of similar magnitude but no longer significant (supplemental Table $3)$.

\section{Publication and availability biases}

A check for potential publication bias was made for PR using $20 \mu \mathrm{M}$ ADP, on which the main analyses were performed. A funnel plot was obtained by representing the HR of PR using 20 
$309 \mu \mathrm{M}$ ADP and the standard error, assessed in each separate study (supplemental Figure 4).

310 Two studies with a negative association between PR using $20 \mu \mathrm{M}$ ADP and the risk of MACE

311 (with small sample sizes) were detected as missing using the 'trim and fill' method for making

312 the funnel plot symmetrical. When these missing studies were added, the pooled HR was not

313 significantly modified. These findings suggested that the publication bias in our meta-analysis

314 was minor.

315 Seven qualifying studies could not provide individual patient data. It is of note that in five of

316 these, the relation between clopidogrel non-response and ischemic events was not a study

317 objective (pharmacokinetic-pharmacodynamic studies or randomized trials of different

318 clopidogrel loading doses). The two remaining studies ( $n=101$ and 111 patients) were

319 specifically interested in the prognostic value of PR for MACE.

320 
321 Discussion

322 In the present meta-analysis of individual patient data conducted in a representative panel of 323 clopidogrel-treated patients we demonstrated that the association between PR and the risk of 324 MACE depended strongly on the level of cardiovascular risk. When using $20 \mu \mathrm{M}$ ADP, the 325 most commonly used concentration in LTA, the risk of MACE associated with PR increased 326 with the level of cardiovascular risk. Indeed, PR did not affect the risk of MACE in patients 327 presenting no risk factors, however it gradually increased the risk of MACE as the number of 328 cardiovascular risk factors increased, reaching a 3.7 times greater risk in high-risk patients 329 with a high PR. The measure of PR with $20 \mu \mathrm{M}$ ADP, in addition to risk factors, modified the 330 interpretation of the 6-month risk of MACE in $44 \%$ of patients, mainly in patients with at least 331 one risk factor.

332 Interestingly, smoking and hypercholesterolemia were not associated with the outcome of 333 MACE and were not included in the analysis of the interaction between PR and risk factors. 334 In randomized controlled trials, the benefit of clopidogrel in reducing the incidence of MACE 335 is primarily seen in smokers, with little benefit to non-smokers(39). With regard to the cohort 336 studies of clopidogrel-treated patients included in this meta-analysis, this differential effect 337 suggests that the increased risk of MACE related to smoking is offset by the benefit 338 clopidogrel provides to smokers; it thereby weakens any possible analysis of the interaction 339 between smoking and PR for outcomes of MACE. Regarding hypercholesterolemia, this 340 conventional risk factor is likely to be confounded by indications for statin treatment. Indeed, 341 in the ADAPT-DES registry(16) hyperlipidemia was protective against mortality with a $342 \mathrm{HR}=0.60(0.41-0.86)$ and was not prognostic of MACE in post-ACS patients with optimal 343 medical therapy(40). In addition, hypercholesterolemia was not homogeneously defined 344 across the studies in the present meta-analysis and other markers, such as plasma LDL345 cholesterol levels, were not widely available.

346 When PR was evaluated using $5 \mu \mathrm{M}$ ADP, its interaction with the level of cardiovascular risk 347 for the prediction of MACE was of a similar magnitude, although non-significant. These 348 findings may reflect the lower number of patients available in studies using $5 \mu M$ ADP, and a 
corresponding loss of power. Moreover, it was previously shown that ADP-induced platelet aggregation in citrated plasma was dependent on the artifactual generation of TxA2 that was modulated by aspirin, at least at lower ADP concentrations(41). This may be associated with an additional background noise in which the interaction between the identified risk factors and PR to predict MACE is blurred, as seen with the lowest $5 \mu \mathrm{M}$ ADP concentrations and partially also with the intermediate $10 \mu \mathrm{M}$ ADP concentrations. Only four of the studies analysed used $10 \mu \mathrm{M}$ ADP, and two of these had a follow-up limited to 30 days; with only 124 MACEs during follow-up, this accounts for a limitation in power to reliably study interactions. Overall, the concentration of ADP used is of limited significance since the influence of risk factors appears in all three ADP concentration groups (table 3 and figure 2). Which laboratory assay and which platelet agonist concentration are best suited for the clinical evaluation of platelet function is the matter of some debate. ADP-induced LTA is highly reproducible within a given laboratory, but its lack of standardization across studies may have slightly weakened the positive findings or lower the level of significance for the interactions found in the present meta-analysis. Of note, the present meta-analysis does not aim to promote the use of LTA to tailor antiplatelet therapy but it rather relied on a historical gold standard in platelet function testing to evidence an interaction with patients' characteristics that should be considered for a tailored approach. The point-of care VerifyNow $\mathrm{P}_{2} \mathrm{Y}_{12}$ assay, used in several intervention trials, correlates well with ADP-induced $\operatorname{LTA}(42,43)$ and we speculate that the main findings of the present meta-analysis would have been similar, had PR been evaluated using the VerifyNow $P 2 Y_{12}$ assay.

Several intervention trials have compared conventional clopidogrel treatment to an antiplatelet strategy tailored according to PR. Early, small randomized trials $(11,12)$ that utilized vasodilator-stimulated phosphoprotein phosphorylation level measurement to indicate $\mathrm{P} 2 \mathrm{Y}_{12}$ receptor reactivity, showed a protective effect for repeat $600 \mathrm{mg}$ clopidogrel loading doses in ACS patients prior to $\mathrm{PCl}$. However, recent larger trials utilizing the VerifyNow $\mathrm{P}_{2} \mathrm{Y}_{12}$ assay were negative. Indeed, the GRAVITAS(13) and ARCTIC(14) studies failed to show the benefit of a PR-tailored antiplatelet strategy after PCI. Various limitations of these trials were 
addressed in a recent consensus publication(22). The event rate of the GRAVITAS study was low compared to the one used for power calculation, and the antiplatelet effect of the high-dose regimen may have been suboptimal as it reduced the prevalence of high PR by only $22 \%$. Similarly, the ARTIC study population was also at a low absolute risk of subsequent cardiovascular events because the prevalence of ACS patients was low, and the composite endpoint also included other events that may not be related to platelet function. The interaction of PR and the number of risk factors, as identified in the present metaanalysis, substantiates the hypothesis that the risk associated with high PR was not clinically relevant in low-risk patients, and that any measure aiming to lower PR is unlikely to lead to a beneficial reduction of MACE for these low-risk patients. Based on these observations we speculate that higher risk patients are more likely to benefit from a therapy tailored to their initial PR. This may explain why early interventions designed to efficiently blunt high PR in ACS patients with multiple conventional risk factors translated into a reduction of MACE(11, 12, 22).

In the current new antiplatelet era, prasugrel and ticagrelor have a major part to play in the management of ACS, leaving clopidogrel as an alternative for patients with high bleeding risk. However, a recent cost-effectiveness analysis for six European perspectives showed that the universal use of newer $\mathrm{P}_{2} \mathrm{Y}_{12}$ inhibitors for ACS patients is probably not as cost-effective as strategies based on $\mathrm{PR}(44)$. It should also be kept in mind that ticagrelor and prasugrel increase the risk of bleeding and that a therapeutic medium-PR window is associated with optimal net clinical benefit(22). The net benefits of newer $P 2 Y_{12}$ inhibitors could also probably be improved not only by testing for PR, but also by incorporating patient risk levels in the decision-making process. Although ongoing trials on tailored $\mathrm{P}_{2} \mathrm{Y}_{12}$ strategies, including TROPICAL-ACS (ClinicalTrials.gov identifier: NCT01959451) and ANTARCTIC(45) partly include this concept of risk levels, further efforts in this direction are needed. This meta-analysis has several strengths, such as the good overall quality of the studies included, as assessed using a quality tool specifically adapted to prognostic studies. The availability of individual patient data allowed a reliable evaluation of the risk associated with 
PR and of the interaction with vascular risk factors. Readily available risk factors relevant to a 406 secondary prevention population were thus identified. The consistency of results across the 407 different ADP concentrations used in the different studies to assess PR, as well as the

409 Despite the advantages related to the availability of individual patient data, this meta-analysis 410 also had some limitations, including a low proportion of women (25\%). This did not allow a 411 stratification of the analyses by gender, as is usually the case in risk assessment tools such 412 the European SCORE or the Framingham risk score. Indeed, in these latter scores gender is 413 not considered as one of traditional risk factors, but is rather presented in separate charts for 414 women and men. There were incomplete data on concomitant medications or other relevant 415 risk factors such as the left ventricular ejection fraction, cholesterol levels or renal 416 insufficiency. Finally, information on bleeding was limited to five studies and a low number of 417 events, thus precluding a reliable analysis of bleeding events and their relation to PR. 418 In conclusion, high PR in patients on clopidogrel is associated with an increased risk of 419 MACE in patients with vascular risk factors, but not in low-risk patients. These findings 420 suggest that trials on tailored PR treatment strategies should be primarily stratified on the 421 individual vascular risk factors in order to assess a truly personalized approach.

423 Funding statement: French ministry of health PHRC 15-07 to JL Reny "Etudes prospectives 424 sur la réponse biologique au clopidogrel et évènements ischémiques chez les patients 425 athérothrombotiques : Métaanalyse sur données individuelles et résumées" (http://www.plan426 alzheimer.gouv.fr/IMG/pdf/Liste des dossiers retenus - 2 mai 2008.pdf ) and the Geneva 427 University Hospitals. Conducted within the Geneva Platelet Group and the division of Clinical 428 Epidemiology. The funders had no role in study design, data collection and analysis, decision 429 to publish, or preparation of the manuscript.

\section{Acknowledgments:}


432 We thank P Whiting for advices on the evaluation of the quality using the PROBAST tool:

433 The PROBAST tool for the assessment of risk of bias and concerns about applicability for 434 prognostic studies was kindly provided by the PROBAST steering group. This tool is still in 435 development and is available from the PROBAST team (www.systematic-reviews.com/probast).

437 Author contributions:

438 Reny JL, Fontana $\mathrm{P}$, and Combescure $\mathrm{C}$ are guarantors for the study, had full access to the 439 data and take responsibility for the integrity of the data and the accuracy of its analysis 440 Study concept and design: Reny JL, Fontana P, and Combescure C 441 Acquisition of data: Reny JL, Fontana P, Hochholzer W, Neumann FJ, Ten Berg J, Janssen 442 PW, Geisler T, Gawaz M, Marcucci R, Gori AM, Cuisset T, Alessi MC, Berdagué P, Gurbel P, 443 Yong G, Angiolillo D, Aradi D, Beigel R, Campo G.

444 Data management and statistical analysis: Combescure C and Reny JL 445 Drafting and critical revision of the manuscript for important intellectual content:: Reny JL, 446 Fontana P, Hochholzer W, Neumann FJ, Ten Berg J, Janssen P, Geisler T, Gawaz M, 447 Marcucci R, Gori AM, Cuisset T, Alessi MC, Berdagué P, Gurbel P, Yong G, Angiolillo D, 448 Aradi D, Beigel R, Campo G, Combescure C.

449 All authors had full access to all of the data (including statistical reports and tables) in the 450 study and can take responsibility for the integrity of the data.

\section{Conflicts of interest:}

453 Reny JL: payment for lectures by Merck Sharp and Dohme

454 Fontana P: consultancy for Evolva; grants from Evolva and Astra Zeneca, payment for

455 lectures by Bayer, AstraZeneca.

456 Hochholzer W: no conflicts of interest.

457 Neumann FJ: no conflicts of interest

458 Ten Berg J: consultancy for Astra Zeneca, Eli Lilly, Merck, and Daiichi Sankyo

459 Janssen P: no conflicts of interest 
Geisler T: consultancy for Bayer, Medicines company, Eli Lilly, Pfizer, BMS, and Daiichi

461 Sankyo; payments for lectures by Bayer, Medicines company, Eli Lilly, Pfizer, BMS, and

462 Daiichi Sankyo; MSD, Boehringer, Astra Zeneca.

463 Gawaz M: consulting fee for Bayer, Astra Zeneca, MSD. Lilly; consultancy for Boehringer-

464 Ingelheim

465 Marcucci R: no conflicts of interest

466 Gori AM: no conflicts of interest

467 Cuisset T: no conflicts of interest

468 Alessi MC: Board membership for Astra Zeneca and Lilly; lectures for Roche;

469 Berdagué P: no conflicts of interest

470 Gurbel P: Served as a consultant for Daiichi Sankyo, Sankyo, Lilly, Bayer, AstraZeneca, 471 Accumetrics, Merck, Medtronic, CSL, and Haemonetics; receiving grants from the National 472 Institutes of Health, Daiichi Sankyo, Lilly, CSL, AstraZeneca, Harvard Clinical Research 473 Institute, Haemonetics, and Duke Clinical Research Institute; receiving payment for lectures, 474 including service on speakers' bureaus, from Lilly, Daiichi Sankyo, and Merck; receiving 475 payment for development of educational presentations from Merck, the Discovery Channel, 476 and Pri-Med; Dr. Gurbel holds stock or stock options in Merck, Medtronic, and Pfizer; and 477 holds patents in the area of personalised antiplatelet therapy and interventional cardiology. 478 Yong G: no conflicts of interest

479 Angiolillo DJ: Received payment as an individual for: a) Consulting fee or honorarium from 480 Bristol-Myers Squibb, Sanofi-Aventis, Eli Lilly, Daiichi Sankyo, Inc., The Medicines Company, 481 AstraZeneca, Merck, Evolva, Abbott Vascular, and PLx Pharma; b) Participation in review 482 activities from Johnson \& Johnson, St. Jude, and Sunovion ; c) has received institutional 483 payments for grants from Bristol-Myers Squibb, Sanofi-Aventis, GlaxoSmith Kline, Otsuka, 484 Eli Lilly, Daiichi Sankyo, Inc., The Medicines Company, AstraZeneca, Evolva, Gilead; and 485 has other financial relationships with Esther and King Biomedical Research Grant. 486 Aradi D: consultancy for Verum Diagnostica $\mathrm{GmbH}$; lectures for Verum Diagnostica, Roche, 487 DSI/Lilly, Bayer, Astra-Zeneca, Pfizer, Biotronic, Abbott. 
1
2
3
4
5
6
7
8
9

6488 Beigel R: no conflicts of interest

7489 Campo G: no conflicts of interest

9490 Combescure C: no conflicts of interest 10

11

12

13

14

15

16

17

18

19

20

21

22

23

24

25

26

27

28

29

30

31

32

33

34

35

36

37

38

39

40

41

42

43

44

45

46

47

48

49

50

51

52

53

54

55

56

57

58

59

60 


\section{References}

492 1. Eikelboom JW, Hirsh J, Spencer FA, et al. Antiplatelet drugs: Antithrombotic Therapy 493 and Prevention of Thrombosis, 9th ed: American College of Chest Physicians Evidence494 Based Clinical Practice Guidelines. Chest 2012; 141(2 Suppl): e89S-119S.

495 2. Yusuf S, Zhao F, Mehta SR, et al. Effects of clopidogrel in addition to aspirin in 496 patients with acute coronary syndromes without ST-segment elevation. N Engl J Med 2001; 497 345(7): 494-502.

498 3. Gurbel PA, Bliden KP, Hiatt BL, et al. Clopidogrel for coronary stenting: response 499 variability, drug resistance, and the effect of pretreatment platelet reactivity. Circulation 2003; 500 107(23): 2908-13.

501 4. Hochholzer W, Trenk D, Frundi D, et al. Time dependence of platelet inhibition after a 502 600-mg loading dose of clopidogrel in a large, unselected cohort of candidates for 503 percutaneous coronary intervention. Circulation 2005; 111(20): 2560-4.

504 5. Snoep JD, Hovens MM, Eikenboom JC, et al. Association of laboratory-defined 505 aspirin resistance with a higher risk of recurrent cardiovascular events: a systematic review 506 and meta-analysis. Arch Intern Med 2007; 167(15): 1593-9.

507 6. Aradi D, Komocsi A, Vorobcsuk A, et al. Prognostic significance of high on-clopidogrel 508 platelet reactivity after percutaneous coronary intervention: systematic review and meta509 analysis. Am Heart J 2010; 160(3): 543-51.

$5107 . \quad$ Combescure C, Fontana P, Mallouk N, et al. Clinical implications of clopidogrel non511 response in cardiovascular patients: a systematic review and meta-analysis. J Thromb 512 Haemost 2010; 8(5): 923-33.

513 8. Reny JL, Berdague P, Poncet A, et al. Antiplatelet Drug Response Status Does Not 514 Predict Recurrent Ischemic Events in Stable Cardiovascular Patients: Results of the 515 Antiplatelet Drug Resistances and Ischemic Events Study. Circulation 2012; 125(25): 320151610.

517 9. Pettersen AA, Seljeflot I, Abdelnoor M, et al. High On-Aspirin Platelet Reactivity and 518 Clinical Outcome in Patients With Stable Coronary Artery Disease: Results From ASCET 
519 (Aspirin Nonresponsiveness and Clopidogrel Endpoint Trial). Journal of the American Heart 520 Association 2012; 1(3): e000703.

521 10. Gurbel PA, Erlinge D, Ohman EM, et al. Platelet function during extended prasugrel 522 and clopidogrel therapy for patients with ACS treated without revascularization: the 523 TRILOGY ACS platelet function substudy. JAMA 2012; 308(17): 1785-94.

524 11. Bonello L, Camoin-Jau L, Arques S, et al. Adjusted clopidogrel loading doses 525 according to vasodilator-stimulated phosphoprotein phosphorylation index decrease rate of 526 major adverse cardiovascular events in patients with clopidogrel resistance: a multicenter 527 randomized prospective study. J Am Coll Cardiol 2008; 51(14): 1404-11.

528 12. Bonello L, Camoin-Jau L, Armero S, et al. Tailored clopidogrel loading dose 529 according to platelet reactivity monitoring to prevent acute and subacute stent thrombosis. 530 Am J Cardiol 2009; 103(1): 5-10.

531 13. Price MJ, Berger PB, Teirstein PS, et al. Standard- vs high-dose clopidogrel based on 532 platelet function testing after percutaneous coronary intervention: the GRAVITAS 533 randomized trial. JAMA 2011; 305(11): 1097-105.

534 14. Collet JP, Cuisset T, Range G, et al. Bedside monitoring to adjust antiplatelet therapy 535 for coronary stenting. N Engl J Med 2012; 367(22): 2100-9.

536 15. Aradi D, Komocsi A, Price MJ, et al. Efficacy and safety of intensified antiplatelet 537 therapy on the basis of platelet reactivity testing in patients after percutaneous coronary 538 intervention: systematic review and meta-analysis. Int J Cardiol 2013; 167(5): 2140-8.

539 16. Stone GW, Witzenbichler B, Weisz G, et al. Platelet reactivity and clinical outcomes 540 after coronary artery implantation of drug-eluting stents (ADAPT-DES): a prospective 541 multicentre registry study. Lancet 2013; 382(9892): 614-23.

542 17. Brar SS, ten Berg J, Marcucci R, et al. Impact of platelet reactivity on clinical 543 outcomes after percutaneous coronary intervention. A collaborative meta-analysis of 544 individual participant data. J Am Coll Cardiol 2011; 58(19): 1945-54. 

testing on clinical outcomes of patients with cardiovascular disease: a systematic review and meta-analysis. Platelets 2013; 24(5): 352-61.

548 19. Moher D, Pham B, Klassen TP, et al. What contributions do languages other than 549 English make on the results of meta-analyses? J Clin Epidemiol 2000; 53(9): 964-72.

550 20. Berlin JA. Does blinding of readers affect the results of meta-analyses? University of 551 Pennsylvania Meta-analysis Blinding Study Group. Lancet 1997; 350(9072): 185-6.

552 21. Bonello L, Tantry US, Marcucci R, et al. Consensus and future directions on the 553 definition of high on-treatment platelet reactivity to adenosine diphosphate. J Am Coll Cardiol 554 2010; 56(12): 919-33.

555 22. Tantry US, Bonello L, Aradi D, et al. Consensus and update on the definition of on556 treatment platelet reactivity to adenosine diphosphate associated with ischemia and bleeding. 557 J Am Coll Cardiol 2013; 62(24): 2261-73.

558 23. Stewart GB, Altman DG, Askie LM, et al. Statistical analysis of individual participant 559 data meta-analyses: a comparison of methods and recommendations for practice. PLoS One $560 \quad 2012 ; 7(10):$ e46042.

561 24. Akritas MG. Nearest neighbor estimation of a bivariate distribution under random 562 censoring. Ann Stat 1994; 22: 1299-327.

563 25. Pencina MJ, D'Agostino RB, Sr., Steyerberg EW. Extensions of net reclassification 564 improvement calculations to measure usefulness of new biomarkers. Stat Med 2011; 30(1): 565 11-21.

566 26. Stroup DF, Berlin JA, Morton SC, et al. Meta-analysis of observational studies in 567 epidemiology: a proposal for reporting. Meta-analysis Of Observational Studies in 568 Epidemiology (MOOSE) group. JAMA 2000; 283(15): 2008-12.

569 27. Campo G, Valgimigli M, Gemmati D, et al. Value of Platelet Reactivity in Predicting 570 Response to Treatment and Clinical Outcome in Patients Undergoing Primary Coronary 571 Intervention. Insights Into the STRATEGY Study. Journal of the American College of 572 Cardiology 2006; 48(11): 2178-85. 
573 28. Hochholzer W, Trenk D, Bestehorn HP, et al. Impact of the degree of peri574 interventional platelet inhibition after loading with clopidogrel on early clinical outcome of 575 elective coronary stent placement. J Am Coll Cardiol 2006; 48(9): 1742-50.

576 29. Angiolillo DJ, Bernardo E, Sabate M, et al. Impact of platelet reactivity on cardiovascular outcomes in patients with type 2 diabetes mellitus and coronary artery disease. J Am Coll Cardiol 2007; 50(16): 1541-7.

30. Cuisset T, Frere C, Quilici J, et al. High post-treatment platelet reactivity is associated with a high incidence of myonecrosis after stenting for non-ST elevation acute coronary syndromes. Thrombosis and haemostasis 2007; 97(2): 282-7.

31. Geisler T, Grass D, Bigalke B, et al. The Residual Platelet Aggregation after Deployment of Intracoronary Stent (PREDICT) score. J Thromb Haemost 2008; 6(1): 54-61.

32. Gurbel PA, Antonino MJ, Bliden KP, et al. Platelet reactivity to adenosine diphosphate and long-term ischemic event occurrence following percutaneous coronary intervention: a potential antiplatelet therapeutic target. Platelets 2008; 19(8): 595-604.

33. Cuisset T, Frere C, Quilici J, et al. Predictive values of post-treatment adenosine diphosphate-induced aggregation and vasodilator-stimulated phosphoprotein index for stent thrombosis after acute coronary syndrome in clopidogrel-treated patients. Am J Cardiol 2009; 104(8): 1078-82.

34. Yong G, Rankin J, Ferguson L, et al. Randomized trial comparing 600- with 300-mg loading dose of clopidogrel in patients with non-ST elevation acute coronary syndrome undergoing percutaneous coronary intervention: results of the Platelet Responsiveness to Aspirin and Clopidogrel and Troponin Increment after Coronary intervention in Acute coronary Lesions (PRACTICAL) Trial. Am Heart J 2009; 157(1): 60 e1-9.

35. Breet NJ, van Werkum JW, Bouman HJ, et al. Comparison of platelet function tests in predicting clinical outcome in patients undergoing coronary stent implantation. JAMA 2010; 303(8): 754-62.

36. Marcucci R, Gori AM, Paniccia R, et al. High on-treatment platelet reactivity by more than one agonist predicts 12-month follow-up cardiovascular death and non-fatal myocardial 
601 infarction in acute coronary syndrome patients receiving coronary stenting. Thromb Haemost 602 2010; 104(2): 279-86.

603 37. Beigel R, Hod H, Fefer P, et al. Relation of aspirin failure to clinical outcome and to 604 platelet response to aspirin in patients with acute myocardial infarction. Am J Cardiol 2011; 605 107(3): 339-42.

606 38. Aradi D, Rideg O, Vorobcsuk A, et al. Justification of $150 \mathrm{mg}$ clopidogrel in patients 607 with high on-clopidogrel platelet reactivity. Eur J Clin Invest 2012; 42(4): 384-92.

608 39. Gagne JJ, Bykov K, Choudhry NK, et al. Effect of smoking on comparative efficacy of 609 antiplatelet agents: systematic review, meta-analysis, and indirect comparison. BMJ 2013; $610347:$ f5307.

611 40. Lafitte M, Pucheu Y, Latry K, et al. Predictors of cardiovascular prognosis in patients 612 receiving optimized secondary prevention measures after acute coronary syndrome. 613 European journal of preventive cardiology 2013; 20(2): 283-90.

614 41. Cattaneo M. Aspirin and clopidogrel: efficacy, safety, and the issue of drug resistance. 615 Arterioscler Thromb Vasc Biol 2004; 24(11): 1980-7.

616 42. Jeong YH, Bliden KP, Antonino MJ, et al. Usefulness of the VerifyNow P2Y12 assay 617 to evaluate the antiplatelet effects of ticagrelor and clopidogrel therapies. Am Heart J 2012; 618 164(1): 35-42.

619 43. Van Werkum J, Van Der Stelt CA, Seesing TH, et al. A head-to-head comparison 620 between the VerifyNow P2Y12 assay and light transmittance aggregometry for monitoring 621 the individual platelet response to clopidogrel in patients undergoing elective percutaneous 622 coronary intervention. J Thromb Haemost 2006; 4(11): 2516-8.

623 44. Coleman $\mathrm{Cl}$, Limone BL. Universal versus platelet reactivity assay-driven use of 624 P2Y12 inhibitors in acute coronary syndrome patients: cost-effectiveness analyses for six 625 European perspectives. Thromb Haemost 2014; 111(1): 103-10.

626 45. Cayla G, Cuisset T, Silvain J, et al. Platelet function monitoring in elderly patients on 627 prasugrel after stenting for an acute coronary syndrome: Design of the randomized antarctic 628 study. American heart journal 2014; 168(5): 674-81. 


1
2
3
4

2

3

4

5

$6 \quad 629$

7630

9

10

11

12

13

14

15

16

17

18

19

20

21

22

23

24

25

26

27

28

29

30

31

32

33

34

35

36

37

38

39

40

41

42

43

44

45

46

47

48

49

50

51

52

53

54

55

56

57

58

59

60 
631 Table 1. Main characteristics of published studies

\begin{tabular}{|l|c|c|c|c|c|c|c|c|c|c|c|c|c|}
\hline \multicolumn{1}{|c|}{ Study } & $\begin{array}{c}\text { Years of } \\
\text { publication }\end{array}$ & $\begin{array}{c}\text { Patients } \\
(\mathrm{n})\end{array}$ & $\begin{array}{c}\text { Age } \\
(\mathrm{y})\end{array}$ & $\begin{array}{c}\text { Male } \\
(\%)\end{array}$ & $\begin{array}{c}\text { Diabetics } \\
(\%)\end{array}$ & $\begin{array}{c}\text { Smokers } \\
(\%)\end{array}$ & $\begin{array}{c}\text { Hyper- } \\
\text { tension } \\
(\%)\end{array}$ & $\begin{array}{c}\text { Hypercholes- } \\
\text { terolemia } \\
(\%)\end{array}$ & $\begin{array}{c}\text { ACS at } \\
\text { inclusion } \\
(\%)\end{array}$ & $\begin{array}{c}\text { PCI } \\
(\%)\end{array}$ & $\begin{array}{c}\text { Gpllb/llla } \\
\text { inhibitor } \\
(\%)\end{array}$ & $\begin{array}{c}\text { Follow-up } \\
(\mathrm{months})^{*}\end{array}$ & $\begin{array}{c}\text { ADP } \\
(\mu \mathrm{M})\end{array}$ \\
\hline Campo et al.(27) & 2006 & 70 & $64 \pm 13$ & 69 & 19 & 37 & 63 & 34 & 100 & 100 & 100 & $10(15)$ & 5,20 \\
\hline Hochholzer et al.(28) & 2006 & 765 & $66 \pm 9$ & 78 & 24 & 11 & 82 & 92 & 0 & 100 & 0 & $12(12)$ & 5,20 \\
\hline Angiolillo et al.(29) & 2007 & 173 & $67 \pm 9$ & 65 & 100 & 13 & 65 & 68 & 0 & 0 & 0 & $24(36)$ & 20 \\
\hline Cuisset et al.(30) & 2007 & 190 & $65 \pm 12$ & 76 & 33 & 48 & 58 & 53 & 87.4 & 100 & 14.7 & $1(1)$ & 10,20 \\
\hline Geisler et al.(31) & 2008 & 1,092 & $67 \pm 11$ & 74 & 33 & 39 & 80 & 59 & 51.7 & 100 & 7.7 & $1(1)$ & 20 \\
\hline Gurbel et al.(32) & 2008 & 297 & $65 \pm 12$ & 65 & 41 & 55 & 74 & 82 & 0 & 100 & 42 & $24(24)$ & 5,20 \\
\hline Cuisset et al.(33) & 2009 & 598 & $65 \pm 12$ & 78 & 35 & 39 & 56 & 55 & 100 & 100 & 9.9 & $1(1)$ & 10 \\
\hline Yong et al.(34) & 2009 & 248 & $63 \pm 12$ & 71 & 22 & 27 & 53 & 52 & 100 & 55 & 39.7 & $6(21)$ & $5,10,20$ \\
\hline Breet et al.(35) & 2010 & 1,069 & $64 \pm 11$ & 75 & 81 & 11 & 77 & 80 & 0 & 100 & 7.0 & $12(12)$ & 5,20 \\
\hline Marcucci et al.(36) & 2010 & 1,108 & $69 \pm 10$ & 75 & 24 & 23 & 66 & 55 & 100 & 100 & 26.0 & $12(12)$ & 10 \\
\hline Beigel et al.(37) & 2011 & 174 & $59 \pm 12$ & 83 & 27 & 41 & 51 & 45 & 100 & 100 & - & $6(6)$ & 5 \\
\hline Aradi et al.(38) & 2012 & 160 & $62 \pm 9$ & 63 & 38 & 36 & 84 & 50 & 0 & 100 & 0 & $12(12)$ & 5 \\
\hline Reny et al.(8) & 2012 & 534 & $62 \pm 12$ & 82 & 21 & 20 & 56 & 63 & 0 & 0 & 0 & $32(50)$ & 5,20 \\
\hline
\end{tabular}

633 Age, mean \pm standard deviation; CAD, coronary artery disease; ACS, acute coronary syndrome; PCI, percutaneous coronary intervention; ADP,

634 adenosine diphospate concentration used for the evaluation of platelet reactivity

635 * Median (maximum) 
637 Table 2: Multivariate analysis to assess the associations between the risk factors and the composite outcome of MACE. This analysis was 638 conducted on the patients of the 13 studies of the meta-analysis ( $n=6,256$ after exclusion of missing data). MACE were observed in 412 patients.

639 Hazard ratios (HR) greater than one show an increased risk of MACE in patients having the corresponding risk factor.

\begin{tabular}{|l|c|c|l|c|c|}
\hline Factors collected in studies & Adjusted HR [95\% Cl] & $\mathbf{p}$ & Level of risk of MACE ${ }^{*}$ & HR [95\% CI] & $\mathbf{p}$ \\
\hline Current smoking status & $0.92[0.71 ; 1.18]$ & 0.50 & Low risk $(n=579)$ & 1 & \\
\hline Age $>75)$ & $1.56[1.25 ; 1.95]$ & $<0.0001$ & Intermediate risk $(n=2444)$ & $1.61[1.05 ; 2.45]$ & 0.03 \\
\hline Diabetes & $1.58[1.27 ; 1.96]$ & $<0.0001$ & High risk $(n=3435)$ & $2.58[1.69 ; 3.94]$ & $<0.0001$ \\
\hline Hypercholesterolemia & $0.86[0.69 ; 1.06]$ & 0.15 & & & \\
\hline Hypertension & $1.23[0.98 ; 1.54]$ & 0.07 & & & \\
\hline ACS at inclusion & $2.00[1.27 ; 3.16]$ & 0.003 & & & \\
\hline Gender (Male) & $1.11[0.89 ; 1.40]$ & 0.35 & & & \\
\hline
\end{tabular}

640 *: a surrogate for the level of risk was defined as the number of risk factors (among age, diabetes, hypertension, and ACS at inclusion): low risk for 641 no risk factor, intermediate risk for one risk factor and high risk for two or more risk factors). 
644 Table 3: Associations between the ADP induced-aggregation categories and the composite outcome of MACE with adjustment on the factors 645 collected in the studies of the meta-analysis (factors shown in Table 2).

\begin{tabular}{|c|c|c|c|c|c|c|}
\hline & \multicolumn{2}{|c|}{ ADP $20 \mu \mathrm{M}$} & \multicolumn{2}{|c|}{ ADP $10 \mu \mathrm{M}$} & \multicolumn{2}{|l|}{ ADP $5 \mu \mathrm{M}$} \\
\hline & $\mathbf{N}$ & & $\mathbf{N}$ & & $\mathbf{N}$ & \\
\hline Studies & 9 & & 4 & & 8 & \\
\hline Events & 287 & & 124 & & 229 & \\
\hline Patients (after exclusion of missing data) & 4,140 & & 2,077 & & 3,160 & \\
\hline & HR $[95 \% \mathrm{Cl}]$ & p & HR $[95 \% \mathrm{Cl}]$ & p & HR $[95 \% \mathrm{Cl}]$ & p \\
\hline ADP induced-aggregation categories & & $0.0003^{* *}$ & 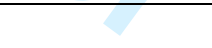 & $0.03^{* *}$ & & $0.02 * *$ \\
\hline Lower category* & 1 & & 1 & ( & 1 & \\
\hline Intermediate category * & $1.85[1.26 ; 2.73]$ & 0.002 & $1.31[0.79 ; 2.17]$ & 0.30 & $1.79[1.02 ; 3.14]$ & 0.04 \\
\hline Higher category* & $2.91[1.78 ; 4.74]$ & $<0.0001$ & $2.61[1.64 ; 4.16]$ & $<0.0001$ & $2.79[1.50 ; 5.22]$ & 0.001 \\
\hline
\end{tabular}

**: global p-values for testing the hypothesis that both HRs (intermediate- and higher-ADP induced-aggregation category) equal 1 
Table 4: Reclassification of the 6-month risk of MACE when the individual risk was predicted from platelet reactivity measured by $20 \mu \mathrm{M}$ ADP in addition to risk factors. The predicted risk was stratified in three levels (low: $\leq 3 \%$, intermediate: $>3 \%$ and $\leq 5 \%$, high: $>5 \%$ ) in agreement with the 6 month risk observed in patients with none, one and two or more risk factors $(2.3 \%, 4.1 \%$ and $6.2 \%$ respectively). Patients were stratified according

to their number of risk factors and to the level of the predicted risk. The numbers of patients and, in brackets, the corresponding observed 6-month risk of MACE in each stratum.

\begin{tabular}{|c|c|c|c|c|}
\hline \multirow[b]{2}{*}{$\begin{array}{l}\text { Risk predicted by the number of risk } \\
\text { factors only }\end{array}$} & \multicolumn{4}{|c|}{$\begin{array}{l}\text { Risk predicted by the combination of risk factors and platelet reactivity measured by } \\
\qquad 20 \mu \mathrm{M} \text { ADP }\end{array}$} \\
\hline & Low risk $(\leq 3 \%)$ & $\begin{array}{l}\text { Intermediate risk }(>3 \% \\
\quad \text { and } \leq 5 \%)\end{array}$ & $\begin{array}{l}\text { High risk } \\
(>5 \%)\end{array}$ & Total \\
\hline Low risk - no risk factor & $524 *\left(2.4 \%{ }^{* *}\right)$ & 26 * & 0 * & $550 *\left(2.3 \%{ }^{* *}\right)$ \\
\hline Intermediate risk - one risk factor & $625^{*}\left(2.1 \%{ }^{* *}\right)$ & $576 *(3.7 \% * *)$ & $\begin{array}{c}6222^{*} \\
(6.3 \% * *\end{array}$ & $1823^{*}\left(4.1 \%{ }^{* *}\right)$ \\
\hline High risk - two or more risk factors & $102 *\left(0.0 \%{ }^{* *}\right)$ & $462 *(3.0 \% * *)$ & $\begin{array}{l}1256^{*} \\
\left(7.6 \% \%^{* *}\right)\end{array}$ & $1820 *\left(6.2 \%{ }^{* *}\right)$ \\
\hline Total & $1251 *\left(2.1 \%{ }^{* *}\right)$ & $1064 *\left(3.4 \%{ }^{* *}\right)$ & $\begin{array}{c}1878^{*} \\
(7.1 \% * *\end{array}$ & $4193 *\left(4.7 \%{ }^{* *}\right)$ \\
\hline
\end{tabular}


659 Figure 1: Flow chart of the meta-analysis

660

\section{1,995 identified references}

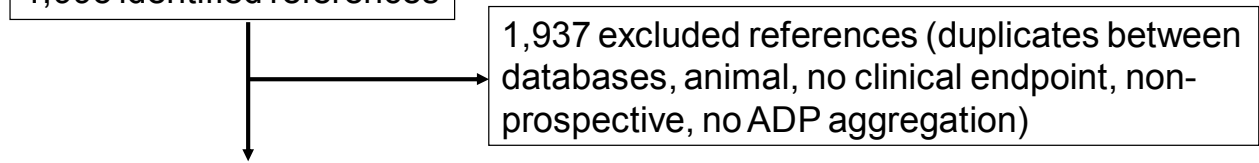

58 full-text articles assessed for eligibility

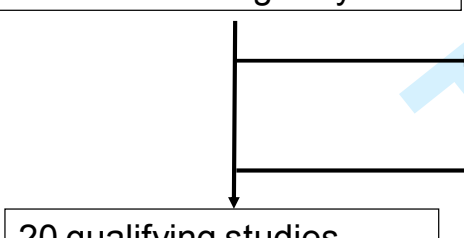

\section{9 duplicate data}

29 excluded studies (non-prospective, no clinical endpoint, no ADP aggregation, non

20 qualifying studies english)

7 excluded studies (5 not responding to requests, 2 refusals; not providing data for a total of 557 patients).

13 included studies totaling 6,478 patients 
662 Figure 2: Kaplan-Meier survival curve for the occurrence of MACE

663

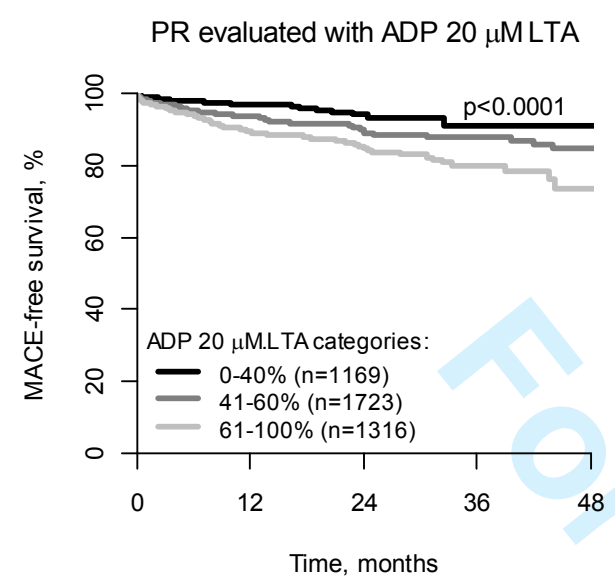

PR evaluated with ADP $10 \mu \mathrm{MLTA}$

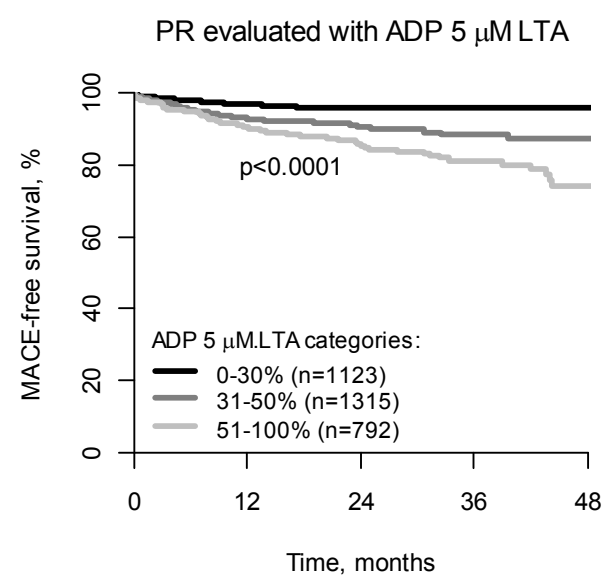


665 Figure 3: Association between platelet reactivity and the occurrence of MACE according to 666 the level of risk. Low-risk patients have none of the risk factors (among age $>75$ years, acute 667 coronary syndrome at inclusion, diabetes, and hypertension), intermediate-risk patients have 668 one risk factor and high-risk patients have two or more risk factors. PR was assessed with 20 $669 \mu \mathrm{M}$ ADP LTA. 670
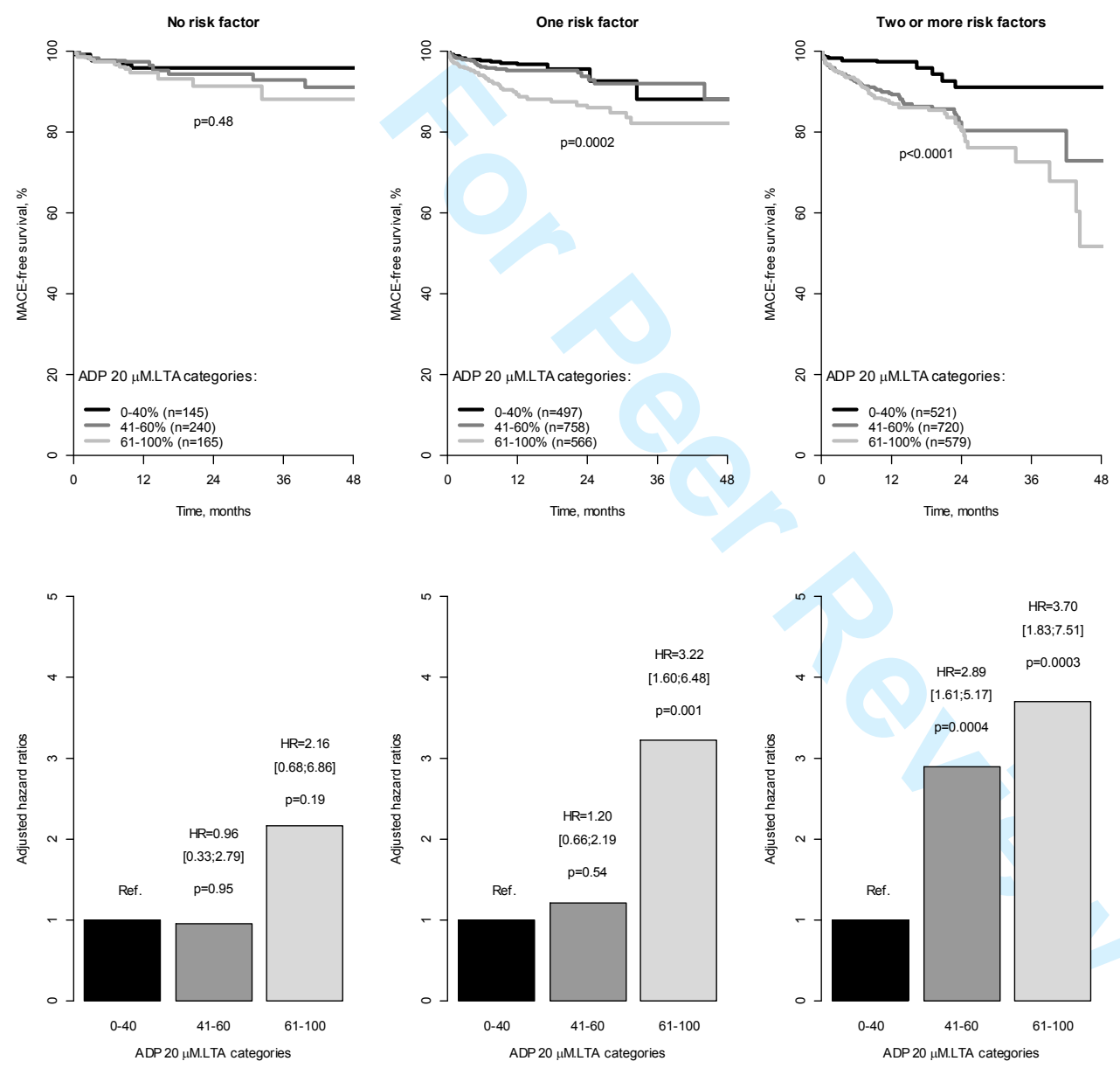
Figure 4: 6-month risk of MACE according to platelet reactivity in the different risk groups. The dashed line represents the overall risk, ignoring platelet reactivity and the black line shows the risk according to the platelet reactivity assessed with $20 \mu \mathrm{M}$ ADP LTA, in patients with no risk factors (A), one risk factor $(B)$ and two or more risk factors (C).
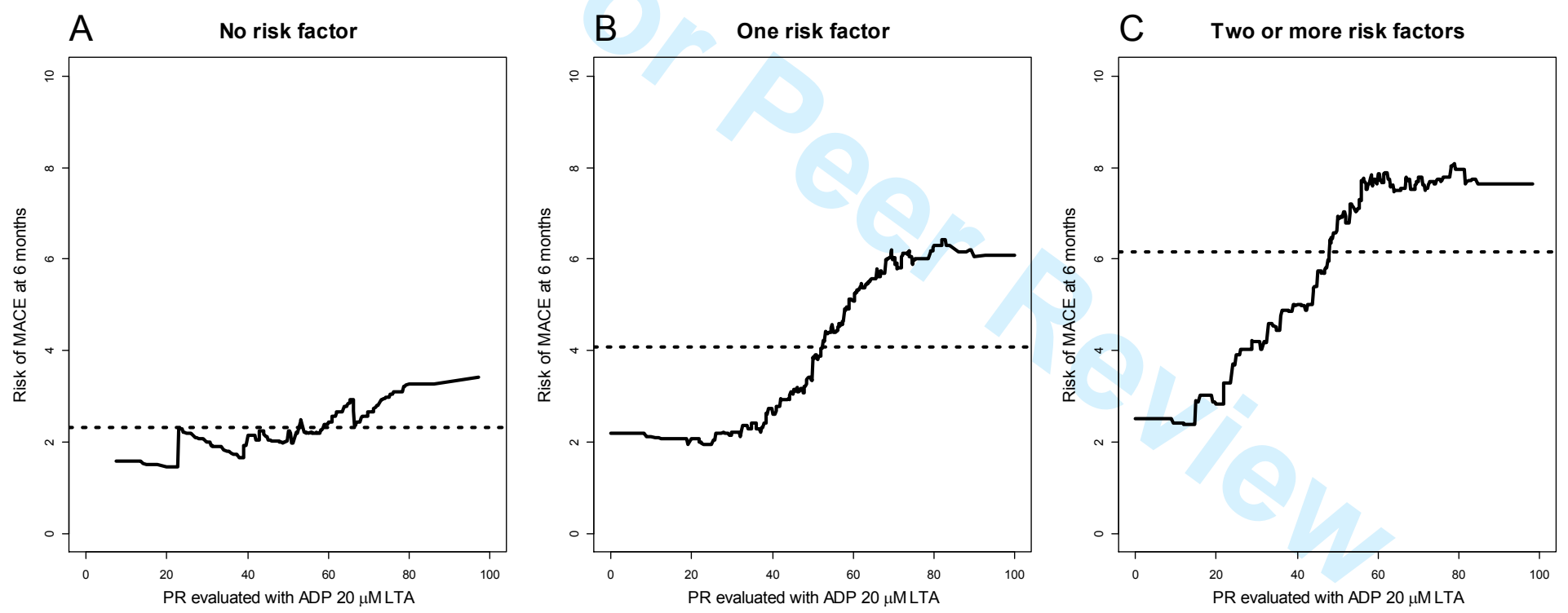
Rebuttal to BMJ reviewers' comments

\section{Reviewer: 1}

Comments:

I think this is a fascinating study performed by experts in the field of platelet function analysis. There is a clear demonstration of high residual platelet reactivity in treated patients with intermediate and high levels of vascular risk factors and its contribution to further adverse events. This paper will help redirect further research in this area and could like to significant health benefits at if it can be demonstrated that changes in therapy provide better healthcare.

Response : NA

\section{Reviewer: 2}

Comments:

In the present paper, the relation between platelet reactivity testing and number of vascular risk factors is studied affecting major adverse cardiovascular events. The study may add to existing literature as intervention studies so far did show the expected benefit in patients with low reactivity.

1. The background of the studies included in this meta analysis should be better clarified: The differential between purely observational cohorts versus studies in patients undergoing revascularization studies. In patients undergoing revascularization the risk for periprocedural adverse outcome is considered especially high in patients with low reactivity but this category is not clearly differentiated from the patients receiving secundairy preventive medical treatment only. Please make this more clear to the reader.

Response : we fully agree with this comment and purposely provided the information in table 1 on patients with ACS and/or PCI at inclusion (a majority of patients). It was likely that in patients with ACS, platelet reactivity would play a more important role but it was, up to now, not clearly established with strong data. The fact that ACS is a risk factor showing an interaction with platelet reactivity toward the outcome of MACE was identified in the present meta-analysis, within the set of risk factors associated with the outcome.

\section{The endpoints are ACS, ischemic stroke and vascular death. Why not including any} death ?

Response : these endpoints are consensual and the widely used so-called MACE or MACCE endpoints. While we agree that it is important to monitor " all deaths » when performing a clinical trial, it may not be relevant to include non-vascular deaths in a composite outcome when a study is interested in platelet reactivity and the risk of recurrent thrombotic events. One can speculate that platelet reactivity could play a role in cancer-related deaths thereby having an impact on total deaths but we were interested in a potential interaction between platelet reactivity and vascular risk factors. In order to avoid any diluting or noise effect due to the inclusion of total deaths we restricted our composite outcome to ischemic events only. Finally, as the outcome items were pre-specified and did not include non-vascular deaths we did not request individual patient data on this latter item and cannot perform this analysis now.

3. Did the authors look at separate outcome parameters within this dataset; especially on ACS versus stroke leaving vascular death out of the perspective?

Response : we did not look at each outcome separately or leaving vascular death aside. As mentioned in the results section, > 90\% of the events were ACS (383 ACS out of 421 MACE), thereby precluding a reliable analysis on separate outcomes. Of note, vascular deaths were adjudicated by an independent adjudicating committee in some of the included studies. 
4. Results page 10 line 47: reporting of stent thrombosis as outcome. This was not indicated as outcome parameter or it should be that ALL thrombosis led to ACS ? please explain.

Response : the information on stent thrombosis is provided as a descriptive statistics. All stent thrombosis indeed led to an ACS as this was mentioned in the manuscript ("There were 83 stent thromboses, including 79 definite or probable and four possible ones, all included in the composite outcome of MACE")

\section{A serious limitation is the use of LTA for PR only. Especially as the authors state in the} introduction that verify now was never tested likewise it would have seemed easy to also look for outcome of verify Now testing. Please explain. Also, make clear in the conclusion section that observed results account for LTA only !

Response: we acknowledge that the conclusion is supported by LTA data only and this was indeed the design of this IPD meta-analysis. We extensively discussed this in the manuscript. The definition of $P R$ is given in the abstract, in the introduction, in the methods, in the results and in the discussion. We feel that this is clearly stated throughout the manuscript and that the conclusion should remain concise.

As mentioned in the Discussion section, LTA is considered the gold against which all other point of care assays were developed. Finally, a meta-analysis on 3059 individual patient data using the VerifyNow assay has been performed, but its power was lower than the present work and the interaction with vascular risk was not investigated (Brar SS, ten Berg J, Marcucci R, Price MJ, Valgimigli M, Kim HS, Patti G, Breet NJ, DiSciascio G, Cuisset T, Dangas G. Impact of platelet reactivity on clinical outcomes after percutaneous coronary intervention. A collaborative meta-analysis of individual participant data. J Am Coll Cardiol. 2011;58:1945-1954)

6. In all risk prediction models in patients with cardiovascular patients age shows the largets effect. please extend more on the relation ship between age as a single risk factor and platelet reactivity.

Response: for reviewing purposes we performed the suggested analysis. The category of platelets reactivity (measured with ADP $20 \mu \mathrm{M} L T A$ ) was not associated with age (Kruskal-Wallis test: $p=0.44$ ): the median (IQR) values were 66.0 years (58.0 to 73.9) in the ADP aggregation category $0-40 \%, 66.0$ years (57.5 to 73.0 ) in the $41-60 \%$ category and 66.2 years (57.5 to 73.3 ) in the $41-100 \%$ category. Distributions of age are shown in the Figure below:

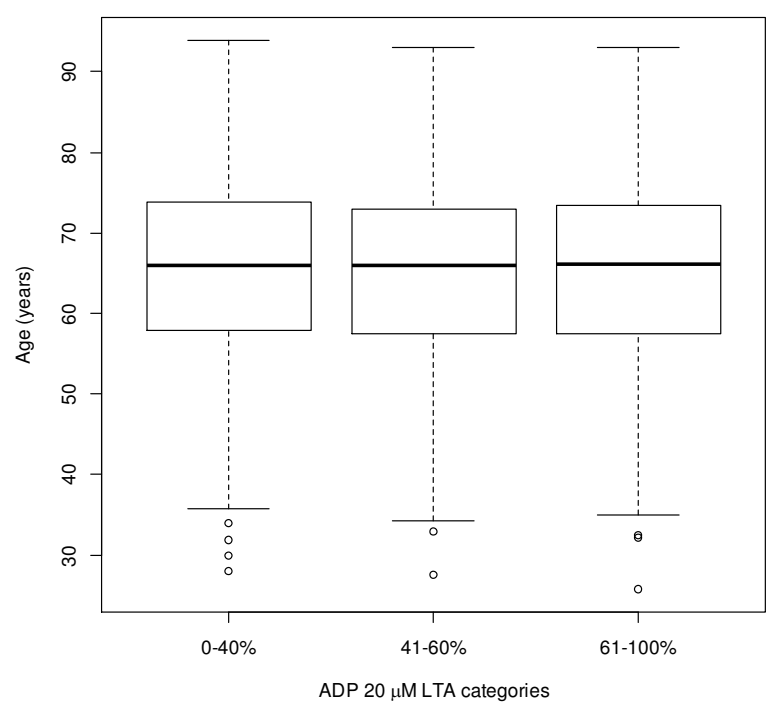

In a univariate Cox regression model with a mixed effect to take the clustering into account, age was significantly associated with the risk of MACE (HR=1.67 for patients above 75 years versus patients below 75 years old, $95 \% \mathrm{Cl} 1.35$ to $2.07, p<0.0001)$. 
In a univariate Cox regression model with a mixed effect to take the clustering into account, the category of platelet reactivity was significantly associated with the risk of MACE. The hazard ratios compared with the reference $0-40 \%$ category were:

- $\quad H R=1.90(95 \% \mathrm{Cl} 1.29$ to $2.80, p=0.001)$ for the $41-60 \%$ category

- $\quad H R=3.11(95 \% \mathrm{Cl} 2.12$ to $4.57, p<0.0001)$ for the $61-100 \%$ category

When the association between MACE and platelet reactivity measured by $20 \mu M$ ADP aggregation was adjusted for age, the hazards ratios for platelet reactivity categories and age of patients were similar:

- $\quad H R=1.88(95 \% \mathrm{Cl} 1.28$ to $2.77, p=0.001)$ for the $41-60 \%$ category

- $\quad H R=3.01(95 \% \mathrm{Cl} 2.05$ to $4.43, p<0.0001)$ for the $61-100 \%$ category

- $\quad H R=1.56(95 \% \mathrm{Cl} 1.20$ to $2.02, p=0.001)$ for age (patients older than 75 years versus patients less than 75 years old)

In patients younger than 75 years, platelet reactivity was significantly associated with the risk of MACE $(p<0.0001)$ and the hazard ratios were:

- $\quad H R=1.55$ (95\% Cl 1.01 to 2.40), $p=0.046$ ) for the category $41-60 \%$

- $\quad H R=2.76(95 \% \mathrm{Cl} 1.80$ to 4.23$), p<0.0001)$ for the category $61-100 \%$

In patients older than 75 years old, platelet reactivity was also associated with risk of MACE $(p=0.0004)$ and the hazard ratios were greater than for patients younger than 75 years old:

- $\quad H R=3.56(95 \% \mathrm{Cl} 1.48$ to 8.56$), p=0.005)$ for the category $41-60 \%$

- $\quad H R=4.15$ (95\% Cl 1.71 to 10.08$), p=0.002$ ) for the category $61-100 \%$

However, the interaction between platelet reactivity and age for prediction of MACE was not statistically significant $(p=0.12)$.

These results confirm the overall robustness of the main interaction finding but we believe that this type of sub-analysis should not be provided as it complicates the message and is not statistically significant.

7. Conclusion; "suggesting that PR tailored strategies may be most effective in higher risk patients" is not warranted based on the data presented in this study. Therefore this statement does not belong to the conclusion.

Response: We agree that this may sound too speculative and this was stated only as a suggestion. In order to remain conservative and avoid any overstatement we deleted this part of the conclusion

\section{Reviewer: 3}

Comments:

This manuscript reports the results of a meta-analysis of individual patient data on the relationship of the risk of major adverse cardiovascular events (MACE) to platelet reactivity and clinical risk factors in patients treated with clopidogrel prior to percutaneous coronary interventions. The study was performed by a team of multinational European investigators with experience in epidemiology and clinical cardiology. The data base analyzed was large (13 studies including 6478 patients), and the methods used were appropriate. The paper is well organized and clearly written. The conclusions are supported by the data.

The results of the study are important. They make a major contribution to a field of investigation that has high clinical relevance, but one that is burdened by methological disputes and conflicting outcomes. The results described provide new understanding of how platelet function data might be more usefully interpreted. A strong virtue of the study is that the results are consistent with clinical data. The paper will be of significant interest to clinicians.

Critique:

1)The number of authors is excessive. Major contributors should be selected or the work presented on behalf of a coalition consistent with BMJ editorial policy.

Response : we very respectfully disagree as all authors had an active participation in the management of their own studies, provided their own individual patient data and actively participated to this meta- 
analysis and this manuscript. We kindly ask the editors to allow the inclusion of 20 authors, all complying with authorship rules, for this manuscript

2)Page 8, paragrapgh 3: the statement that 20 micromolar ADP is better requires some qualification and references. It is reasonable to think that a maximal agonist stimulus would be the most useful, but it is apparent from the data presented that there is no standard that has been uniformly applied.

Response : We fully agree with this comment. Indeed, it was previously shown that ADP-induced platelet aggregation at low concentration (up to 10 microM) in citrated plasma was dependant of the artefactual generation of TXA2 that is sensitive to aspirin (Cattaneo M. Aspirin and clopidogrel: Efficacy, safety, and the issue of drug resistance. Arterioscler Thromb Vasc Biol. 2004;24:1980-1987). This may be associated with an additional background noise in which the interaction between the identified risk factors and PR to predict MACE is blurred, as seen with the lowest concentrations of $A D P$ This was stated in the discussion and the above reference was cited. We have now added a clear mention of the "lowest concentration" as 5 micromolar and the intermediate 10 micromolar.

3)Data presented in Table 3 and in Figure 2 could be interpreted to indicate that the concentration of ADP used is of limited significance since the influence of risk factors appears in all three ADP concentration groups. this should be discussed by the authors

Response : we fully agree and we have added a sentence with this interpretation. The consistency of the interaction independently of ADP concentration further support the main findings of this metaanalysis.

4)Since the bulk of the work is statistical, the paper should receive careful statistical review.

Response : this was done within the BMJ editorial committee including a statistician, Rafael Perera, who did not have any methodological or statistical issues related to this meta-analysis. In addition we provide a detailed description of the methods used and several sensitivity analyses that support the robustness of the findings (supplemental online material). Finely, we included a quality assessment tool, PROBAST, that was recently and specifically designed for meta-analysis or prognostic studies. The PROBAST tool was kindly made available to us by Dr Penny Whitting as acknowledged in the manuscript. 
MOOSE checklist designed for meta-analyses of observational studies (1) in lieu of the PRISMA checklist(2) focused on meta-analyses of randomized and intervention trials.

Vascular risk levels affect the predictive value of platelet reactivity for the occurrence of major adverse cardiovascular events in patients on clopidogrel: Systematic review and collaborative meta-analysis of individual patient data. Reny JL et al.

\section{Reporting of background should include}

Problem definition: lines 127-128 (Introduction)

Hypothesis statement: lines 132-136 (Introduction)

Description of study outcome(s): line 136 (Introduction), lines 192-207 (Methods)

Type of exposure or intervention used: lines 136-137 (Introduction), lines 192-207 (Methods)

Type of study designs used: line 137 (meta-analysis design, Introduction), lines 162-164

(designs od studies included in the meta-analysis, Methods)

Study population: lines 154-155 (Methods)

\section{Reporting of search strategy should include:}

Qualifications of searchers (eg, librarians and investigators): investigators, methods $p 6$ line 149

Search strategy, including time period included in the synthesis and keywords: lines 143151 (Methods)

Effort to include all available studies, including contact with authors: lines 171-173

Databases and registries searched: lines 143-151 (Methods)

Search software used, name and version, including special features used (eg, explosion): databases cited in the section "Methods" only lines 143-145

Use of hand searching (eg, reference lists of obtained articles): lines 148-149 (Methods)

List of citations located and those excluded, including justification: Figure 1 (flow-chart)

Method of addressing articles published in languages other than English: Not applicable.

Method of handling abstracts and unpublished studies: no congress abstract retrieved

Description of any contact with authors: lines 170-181 (Methods)

\section{Reporting of methods should include:}

Description of relevance or appropriateness of studies assembled for assessing the hypothesis to be tested: The relevance of studies was guaranteed by the inclusion criteria. Lines 154-165

Rationale for the selection and coding of data (eg, sound clinical principles or convenience): Individual patient's data. The format of data provided by authors was harmonized (standardization of units) - lines 178-181 (Methods)

Documentation of how data were classified and coded (eg, multiple raters, blinding, and interrater reliability): Data were not extracted from published papers but provided by authors. The list of required variables was provided to authors as well as the definition of the biological and clinical outcomes. Data were checked for completeness and consistency with published reports. Any discrepancies were resolved with the corresponding authors - lines 178-181 (Methods)

Assessment of confounding (eg, comparability of cases and controls in studies where appropriate): the quality of studies, assessed with the PROBAST tool (methods and acknowledgements), included the risk of bias related to the outcome measurement, follow-up of patients and measure of exposure. Lines 185-189. 
Assessment of study quality, including blinding of quality assessors; stratification or regression on possible predictors of study results: assessment of the quality with the PROBAST tool - lines 185-189 (Methods) and supplement Appendix Table 1.

Assessment of heterogeneity: lines 212-215 (Methods) and Supplement Appendix ("Detailed statistical analysis")

Description of statistical methods (eg, complete description of fixed or random effects models, justification of whether the chosen models account for predictors of study results, dose-response models, or cumulative meta-analysis) in sufficient detail to be replicated : lines 209-228 (Methods) and Supplement Appendix (("Detailed statistical analysis”)

Provision of appropriate tables and graphics: No tables, no graphics in the "Methods" section

\section{Reporting of results should include:}

Graphic summarizing individual study estimates and overall estimate: Individual study estimates are not reported because studies were not powered to test the interaction term between the platelet reactivity and the level of risk. Only overall estimates are reported. Tables 2, 3 and section "Results".

Table giving descriptive information for each study included: Table 1

Results of sensitivity testing (eg, subgroup analysis): Sensitivity are summarized lines 353-365 (Results) and detailed further in Supplement Appendix

Indication of statistical uncertainty of findings: $p$ values for testing the interaction term, $95 \%$ confidence interval for all estimates, publication and availability biases assessed in results (lines 367-380, Results)

\section{Reporting of discussion should include:}

Quantitative assessment of bias (eg, publication bias): publication and availability biases assessed in results (lines 367-380, Results and Supplement Appendix Figure 4)

Justification for exclusion (eg, exclusion of non-English-language citations): Line 143 and reference Moher D, Pham B, Klassen TP, et al. What contributions do languages other than English make on the results of meta-analyses? J Clin Epidemiol. 2000;53(9):964-972.

Assessment of quality of included studies: Lines 241-242 (Results) and Supplement appendix Figure 2), lines 459-460 (discussion)

\section{Reporting of conclusions should include:}

Consideration of alternative explanations for observed results: Lines $466-474$

Generalization of the conclusions (ie, appropriate for the data presented and within the domain of the literature review) : abstract and discussion lines 383-384, 463-466

Guidelines for future research: trials on PR tailored strategy warranted in HPR patients, lines 477-479

Disclosure of funding source : Lines 481-486.

1. Stroup DF, Berlin JA, Morton SC, Olkin I, Williamson GD, Rennie D, et al. Meta-analysis of observational studies in epidemiology: a proposal for reporting. Meta-analysis Of Observational Studies in Epidemiology (MOOSE) group. JAMA. 2000;283(15):2008-12. Epub 2000/05/02.

2. Moher D, Liberati A, Tetzlaff J, Altman DG. Preferred reporting items for systematic reviews and meta-analyses: the PRISMA statement. PLoS medicine. 2009;6(7):e1000097. Epub 2009/07/22. 


\section{Vascular risk levels affect the predictive value of platelet reactivity for the occurrence of major adverse cardiovascular events in patients on clopidogrel: Systematic review and collaborative meta-analysis of individual patient data}

\begin{tabular}{|c|c|}
\hline Journal: & $B M J$ \\
\hline Manuscript ID: & Draft \\
\hline Article Type: & Research \\
\hline BMJ Journal: & BMJ \\
\hline Date Submitted by the Author: & $n / a$ \\
\hline Complete List of Authors: & $\begin{array}{l}\text { Reny, Jean-Luc; Geneva University Hospitals, Internal médicine, } \\
\text { rehabilitation and geriatrics } \\
\text { Fontana, Pierre; Geneva University Hospitals, } \\
\text { Hochholzer, Willibald; Universitaets Herzzentrum Freiburg-Bad Krozingen, } \\
\text { Neumann, Franz-Josef; Universitaets Herzzentrum Freiburg-Bad Krozingen, } \\
\text { ten Berg, Jurriën; St Antionius Hospital, Cardiology } \\
\text { Janssen, Paul; St Antionius Hospital, Cardiology } \\
\text { Geisler, Tobias; University Hospital Tübingen, } \\
\text { Gawaz, Meinrad; University Hospital Tübingen, } \\
\text { Marcucci, Rossella; University of Florence, Azienda Ospedaliero } \\
\text { Universitaria Careggi, } \\
\text { Gori, Anna-Maria; University of Florence, Azienda Ospedaliero Universitaria } \\
\text { Careggi, } \\
\text { Cuisset, Thomas; Faculté de Médecine Marseille, } \\
\text { Alessi, Marie-Christine; Faculté de Médecine Marseille, } \\
\text { Berdagué, Philippe; CH Béziers, } \\
\text { Gurbel, Paul; Sinai Center for Thrombosis Research, } \\
\text { Yong, Gerald; Maryland Royal Perth Hospital, } \\
\text { Angiolillo, Domiiick; University of Florida College of Medicine, } \\
\text { Aradi, Daniel; Heart Centre, University of Pécs, Hungary, } \\
\text { Beigel, Roy; Tel-Hashomer, and the Sackler Faculty of Medicine, } \\
\text { Campo, Gianluca; Azienda Ospedaliera Universitaria di Ferrara, } \\
\text { Combescure, Christophe; Geneva University Hospitals, }\end{array}$ \\
\hline Keywords: & $\begin{array}{l}\text { clopidogrel, drug response, platelets, cardiovascular diseases, ischemic } \\
\text { events }\end{array}$ \\
\hline
\end{tabular}




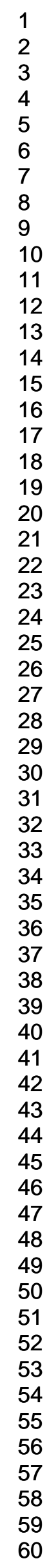

SCHOLARONE $^{\text {Im }}$

Manuscripts

https://mc.manuscriptcentral.com/bmj 
Vascular risk levels affect the predictive value of platelet reactivity for the occurrence

of major adverse cardiovascular events in patients on clopidogrel:

\author{
Systematic review and collaborative meta-analysis of individual patient data
}

Jean-Luc Reny; Pierre Fontana; Willibald Hochholzer; Franz Josef Neumann; Jurrën ten Berg; Paul W.Janssen; Tobias Geisler; Meinrad Gawaz; Rossella Marcucci; Anna-Maria Gori; Thomas Cuisset; Marie-Christine Alessi; Philippe Berdagué; Paul A. Gurbel; Gerald Yong; Dominick J. Angiolillo; Daniel Aradi; Roy Beigel; Gianluca Campo; and Christophe Combescure.

Authors' affiliations:

Geneva Platelet Group, School of Medicine, University of Geneva, Geneva, Switzerland (Dr. Reny, Dr. Fontana); Division of Internal Medicine and Rehabilitation, Trois-Chêne Hospital, Geneva University Hospitals, Geneva, Switzerland (Dr. Reny); Division of Angiology and Haemostasis, Geneva University Hospitals, Geneva, Switzerland (Dr. Fontana); Universitaets Herzzentrum Freiburg-Bad Krozingen, Klinik für Kardiologie und Angiologie II, Bad Krozingen, Germany (Dr. Hochholzer, Dr. Neumann); St Antonius Hospital, Nieuwegein, the Netherlands (Dr. ten Berg, Dr. Janssen); University Hospital Tübingen, Department of cardiology, Tübingen, Germany (Dr. Geisler, Dr. Gawaz) Center for atherothrombotic diseases, Dept Experimental and Clinical Medicine, University of Florence, Azienda Ospedaliero Universitaria Careggi, Florence, Italy (Dr. Marcucci, Ms Gori); Cardiologie Interventionnelle, CHU Timone Unité Inserm UMR 626, Faculté de Médecine Marseille, France (Dr Cuisset); UMR Inserm 1062, inra 1260, université Aix-Marseille, France (Dr Alessi); Division of Cardiology, Beziers Hospital, Béziers, France (Dr Berdagué); Sinai Center for Thrombosis Research, Sinai Hospital of Baltimore, Baltimore, USA (Dr Gurbel); Maryland Royal Perth Hospital, Australia (Dr. Yong); Division of Cardiology, University of Florida College of Medicine, Jacksonville, Florida, USA (Dr. Angiolillo); Department of Cardiology, Heart Center Balatonfüred, Hungary (Dr. Aradi); The Leviev Heart Center, Sheba Medical Center, Tel-Hashomer, and the Sackler Faculty of Medicine, Tel Aviv University, TelAviv, Israel (Dr. Beigel); Cardiovascular Institute, Azienda Ospedaliera Universitaria di Ferrara, Cona (FE), Italy (Dr Campo); Division of Clinical Epidemiology, Geneva University Hospitals, Geneva, Switzerland (Dr. Combescure).

Correspondence:

Jean-Luc Reny

Division of Internal Medicine and Rehabilitation

Trois-Chêne Hospital, Geneva University Hospitals

Ch. du Pont-Bochet 3

$\mathrm{CH}-1226$ Thônex-Geneva

Switzerland

Phone: +41-22-305 6537

Fax: +41-22-305 6115

E-mail: jean-luc.reny@hcuge.ch

Word count: 4'185 words for the manuscript's introduction, methods, results, and discussion (excluding the abstract, references, tables, figures and supplemental data) 


\section{Abstract}

Objective: Prior studies have shown an association between high on-clopidogrel platelet reactivity (PR) and the risk of major adverse cardiovascular events (MACE). However, large intervention trials on PR-tailored treatments have been neutral, possibly owing to the inclusion of patients at low cardiovascular risk. The role and usefulness of PR with regard to levels of cardiovascular risk are unclear. We assessed the clinical relevance of PR in predicting MACE according to patients' cardiovascular risk levels.

Design: Systematic review and meta-analysis of individual patient data on MACE outcomes (acute coronary syndromes, ischemic strokes, and vascular deaths) in relation to PR and its interaction with cardiovascular risk levels. PR was determined using ADP-induced light transmission aggregometry (LTA) with a primary concentration of $20 \mu \mathrm{M}$ ADP and defined as high (>60\% aggregation), medium $(41-60 \%)$ or low $(<41 \%)$. A surrogate for the level of cardiovascular risk was defined as the number of conventional vascular risk factors with homogeneous definitions across studies and identified as predictors of MACE in the metaanalysis. Associations between the number of risk factors, PR strata, and risk of MACE were analysed using multivariate, mixed-effect Cox models. The net reclassification index (NRI) for survival data and the $\%$ of patients reclassified to a different risk level were computed to quantify the contribution of PR testing for the prediction of the 6-month risk of MACE in patients with increasing numbers of traditional risk factors.

Data sources: Medline, Embase, Web of Science, Cochrane Central Register of Controlled Trials) and abstracts from major international meetings held from 2010-2013 (ISTH, AHA, ACC, ESC). Free-text search conducted using an 'ADP' and 'aggregation' and 'clopidogrel' key-word combination. Inclusion criteria: (a) patients treated with clopidogrel and with symptomatic atherothrombosis; (b) response to clopidogrel evaluated using the maximal aggregation value from LTA with 20,10 , or $5 \mu \mathrm{M}$; (c) LTA performed remote from platelet function interfering drugs other than aspirin or clopidogrel; (d) prospective follow-up for MACE for at least 30 days; (e) prospective cohort or a randomised therapeutic trial. 
Corresponding authors of selected studies were contacted to collaborate to the metaanalysis and to provide their individual patient's data.

Results: Thirteen prospective studies totalled 6,478 clopidogrel-treated patients who experienced 421 MACE (6.5\%) during a median follow-up of 12 months. The risk of MACE associated with PR increased differentially according to the number of risk factors present (age $>75$ years, ACS at inclusion, diabetes, and hypertension; interaction $p=0.04$ ): no association to PR in low-risk patients (no risk factor) $(p=0.48) ; 3.2(1.6$ to $6.5, p=0.001$ ) times greater risk of MACE in high PR intermediate-risk patients (one risk factor); 2.9 (1.6 to 5.2, $\mathrm{p}=0.0004)$ and 3.7 (1.8 to $7, p=0.0003$ ) times greater risk of MACE in medium PR and high PR high-risk patients ( $\geq 2$ risk factors). PR allowed the reclassification of $44 \%$ (1837/4193 patients) of the total population to a different risk level for the outcome of MACE, mostly in patients originally identified as intermediate or high risk.

Conclusion: The magnitude of the association between PR and MACE risk is strongly dependant on the level of cardiovascular risk faced by patients on clopidogrel suggesting that PR-tailored strategies may be most effective in higher-risk patients.

Keywords: clopidogrel, drug response, platelets, cardiovascular diseases, ischemic events. 
Atherosclerotic diseases account for more than $40 \%$ of deaths in Western countries, and antiplatelet therapy is a major preventive strategy in this setting. ${ }^{1}$ Clopidogrel, a P2Y 12 receptor blocker, inhibits the activation of platelets by adenosine diphosphate (ADP), and is widely prescribed for secondary prevention in patients with atherosclerotic diseases. When combined with aspirin, clopidogrel is particularly effective in patients with acute coronary syndromes (ACS), ${ }^{2}$ and has proved superior to aspirin alone in several other large randomised controlled trials. The pharmacodynamic response to clopidogrel shows a wide inter-individual variability. ${ }^{34}$ Numerous cohort studies, often performed on patients with acute coronary syndrome (ACS) and/or undergoing percutaneous coronary interventions (PCI), have shown an association between high on-treatment platelet reactivity $(P R)$ and the risk of recurrent major adverse cardiovascular events (MACE) ${ }^{5-7}$ However, recent studies in cohorts of stable cardiovascular outpatients ${ }^{89}$ or in medically managed ACS patients ${ }^{10}$ failed to confirm these results. Several randomised trials aimed at reducing the recurrence of ischemic events have compared standard clopidogrel treatment to a P2Y ${ }_{12}$-inhibitor strategy tailored according to the presence of high PR. Although initial small trials were promising ${ }^{11}{ }^{12}$ more recent larger trials showed no benefit from adjusting clopidogrel doses or switching to prasugrel based on PR testing in low-risk coronary patients undergoing $\mathrm{PCl}^{13}{ }^{14}$ These contrasting results, both from observational studies and randomised intervention trials, may be explained by different patient characteristics including the level of risk, but to date few data substantiate these hypotheses. We previously showed, in a study-level meta-analysis, that the risk of recurrent MACE associated with high PR was greater in studies using Gpllb/llla inhibitors (a marker of high-risk patients) than in studies which did not. ${ }^{7}$ Another meta-regression from a study-level meta-analysis of randomised trials suggested that the higher the incidence of coronary stent thrombosis in a given study, the larger the net clinical benefit from a PR-tailored strategy. ${ }^{15}$ Finally, the ADAPT-DES registry of patients undergoing $\mathrm{PCl}$ showed that high PR was predictive of stent thrombosis mostly in ACS patients, but there was no interaction reported between PR and the presence of an ACS at inclusion. ${ }^{16}$ 
This information suggests the hypothesis that high PR might be more relevant in high-risk populations, but convincing data at the individual level are lacking. To date, the only metaanalysis on individual patient data performed on 6 studies totalling 3,059 patients assessed with the VerifyNow P2Y12 assay did not explore this hypothesis. ${ }^{17}$ Similarly, one of the largest and more recent meta-analysis on 8 studies and 4817 patients did not explore this interaction due to the lack of individual data. ${ }^{18}$ To further investigate this interaction on a larger population we performed a collaborative meta-analysis of individual patient data and focused on the interaction between relevant vascular risk factors and PR, assessed with ADP induced light transmission aggregometry (LTA), in order to better define the risk of MACE. ADP-induced LTA is the assay upon which all $P 2 Y_{12}$ receptor inhibitors have been developed, thus supporting its use in the present meta-analysis. In addition, among several available assays to evaluate PR, LTA is the historical gold standard with which most platelet function assays were compared. 


\section{Methods}

Data sources

Literature review, confined to articles in English, ${ }^{19}$ was based on electronic databases (Medline, Embase, Web of Science, Cochrane Central Register of Controlled Trials) and abstracts from major international meetings held from 2010-2013 (ISTH, AHA, ACC, ESC). A free-text search was conducted using an 'ADP' and 'aggregation' and 'clopidogrel' keyword combination. Articles were selected on the basis of abstracts, before examination of the full text. Reference lists of selected articles were also hand-searched to identify additional relevant reports. Reviewers (JLR and PF) were not blinded to the journal, authors or institutions in the publications as this has been shown to be unnecessary. ${ }^{20}$ The electronic database search was last updated on 31 July, 2013.

\section{Study selection}

Selected studies met the following criteria: (a) patients were treated with clopidogrel and had symptomatic atherothrombosis (clinical signs related to vascular atherothrombotic lesions);

(b) pharmacodynamic response to clopidogrel was evaluated using the maximal aggregation value from LTA on platelet-rich plasma with 20,10 , or $5 \mu \mathrm{M}$ ADP as an agonist; (c) LTA was performed remote from platelet function interfering drugs such as Gpllb/llla inhibitors; (d) patients were prospectively monitored for MACE for at least 30 days, defined using at least one of the following items: acute coronary syndrome (unstable angina, myocardial infarction with/without ST segment elevation), ischemic stroke (acute neurological deficit due to a cerebral infarction), and vascular death; (e) studies involved either a prospective cohort or a randomised therapeutic trial, but one in which treatment was allocated independently of the response to clopidogrel. When studies were suspected of including the same patients, the authors were asked to provide data from the largest possible number of independent patients. The flow of references through the review process is shown in Figure 1. 


\section{Data extraction}

The corresponding authors or principal investigators of eligible studies were contacted and asked to participate in the CLOpidogrel and Vascular ISchemic events - Individual Patient Data (CLOVIS-IPD) meta-analysis group. Investigators provided individual data on: the qualifying cardiovascular condition and clinical setting at inclusion (ACS or stable disease); MACE and date of occurrence during follow-up; platelet reactivity (PR) with ADP 20, 10, and/or $5 \mu \mathrm{M}$ and its timing relative to loading dose of clopidogrel; age, gender, height, and weight; current smoking status, diabetes, hypercholesterolemia, and hypertension; left ventricular ejection fraction; platelet count; PCl; use of Gpllb/llla inhibitors and timing; concomitant medications; and bleeding events and timing during follow-up. Data were checked for completeness and consistency with published reports. Any discrepancies were resolved with the corresponding authors. After format harmonisation, data were compiled for statistical analysis. All studies were approved by their respective institutional review boards.

\section{Quality assessment of studies}

A new quality assessment tool for prognostic studies called PROBAST (see Acknowledgements) was used to estimate risks of bias and concerns about applicability. As PROBAST is not customised for meta-analyses of individual patient data, items were adapted accordingly. Based on the present study's list of relevant criteria, risks of bias, and concerns about applicability are rated as low, unclear, or high. Supplemental Figure 1 shows the list of criteria.

\section{Primary outcomes and measures}

The primary clinical outcome was the occurrence of MACE, as defined above (see Study selection (d)). The primary biological outcome was maximal aggregation with $20 \mu \mathrm{M}$ ADP, as it is a better concentration for analysing the effects of clopidogrel than lower ones. PR was categorised in three strata. The higher cut-offs were selected on the basis of previously 
published cut-offs (59\% to $64 \%$ for $20 \mu \mathrm{M}$ ADP, and $43 \%$ to $46 \%$ for $5 \mu \mathrm{M}$ ADP), ${ }^{21}$ and to keep relatively balanced numbers of patients in each PR categories. Three pre-specified categories allowed a better description of the dose-dependent effects of PR on the risk of MACE compared to the usual dichotomic high and low PR categorization. Three categories were also chosen to better parallel the analysis with a therapeutic PR window that has been associated with optimal net clinical benefit. ${ }^{22}$ A surrogate for the level of cardiovascular risk was defined as the number of factors with homogeneous definitions across studies, and these were markers of MACE in the meta-analysis. The factors were selected from among age, diabetes, hypertension, smoking, hypercholesterolemia, and the presence of an ACS at inclusion (as defined in study selection (d)), and were all provided at the time of inclusion and PR testing.

\section{Statistical analysis}

MACE-free survival curves were derived from individual patient data using the Kaplan-Meier estimator; curves were compared using log-rank tests stratified by study. Associations between conventional risk factors, PR strata, and risk of MACE were analysed using multivariate, mixed-effect Cox models. The amount of heterogeneity was assessed by the size of the random effects $\left(\mathrm{Tau}^{2}\right)$ which is an estimate of the between study variability. ${ }^{23}$ The presence of heterogeneity was tested by comparing models with and without random effects (likelihood ratio test). The interactions between the level of risk and PR strata were tested. MACE-free survival according to $P R$, as a continuous variable, was assessed using the $R$ package prodlim using the symmetrical nearest neighbourhoods method. ${ }^{24}$ Sensitivity analyses were conducted to check the robustness of the findings with respect to: the risks of bias and concerns about the applicability of studies; the definition of MACE, including target vessel revascularisation or $\mathrm{PCl}$ at inclusion, and; the influence of a given specific study. The net reclassification index (NRI) for survival data ${ }^{25}$ was computed to quantify the contribution of PR testing for the prediction of the 6-month risk of MACE in patients with increasing numbers of traditional risk factors. The event and non-event continuous NRIs were reported. 
Potential publication bias was checked for. P-values below 0.05 were considered significant and all tests were two-sided. Published guidelines for meta-analysis of observational studies in epidemiology (MOOSE) and their reporting ${ }^{26}$ were followed. Details on statistical methods are given in the online data supplement.

\section{Results}

Characteristics of included studies

The Figure 1 flow-chart details how 13 of 20 qualifying studies were included, totalling 6,478 patients. $^{87-38}$ Table 1 shows their characteristics. Data on body mass index, concomitant medications, left ventricular ejection fraction, or the occurrence of target and non-target vessel revascularisation during follow-up were only available in some studies. All studies provided individual data allowing a homogeneous definition of MACE, current smoking status, ACS, diabetes (fasting plasma glucose $\geq 7.0 \mathrm{mmol} / \mathrm{l}$, 2-h plasma glucose $\geq 11.1 \mathrm{mmol} / \mathrm{l}$ after $75 \mathrm{~g}$ oral glucose load or background therapy for diabetes), and hypertension (systolic blood pressure $\geq 140 \mathrm{mmHg}$ or diastolic blood pressure $\geq 90 \mathrm{mmHg}$ or a documented history of hypertension). Hypercholesterolemia was not defined in a homogeneous fashion across studies and plasma LDL-cholesterol levels were not available for more than 2,000 patients. Overall, risks of bias and concerns about applicability were low (online data supplement further details study characteristics, bias, and applicability). Information on bleeding was limited to five studies, with only 67 major and 20 moderate/minor bleedings.

\section{MACE and level of risk}

Overall, 421 MACE occurred in 6,478 patients $(6.5 \%)$, the majority being ACS $(n=383)$. There were 83 stent thromboses, including 79 definite or probable and four possible ones, all included in the composite outcome of MACE. The MACE-free survival rate across the different studies at the end of follow-up ranged from $77.4 \%$ to $97.3 \%$. In a multivariate analysis, four factors were found relevant to determining patients' levels of risk: age greater than 75 years, diabetes, ACS at inclusion, and hypertension (Table 2). The number of these 
factors was used as a surrogate for the individual risk of MACE. Patients with none of these factors were classified 'low-risk', patients with one factor 'intermediate-risk', and patients with two or more factors 'high-risk' (global p-value $<0.0001$ for the trend).

\section{MACE and PR}

Nine studies ( $n=4,438$ patients) performed LTA using $20 \mu$ MDP, four studies $(n=2,144$ patients) used $10 \mu \mathrm{M}$ ADP, and eight studies ( $n=3,317$ patients) used $5 \mu \mathrm{M}$ ADP. Figure 2 shows the MACE-free survival curves by category of ADP concentration. Risk of MACE increased significantly with PR with $20 \mu \mathrm{M}$ ADP, $10 \mu \mathrm{M}$ ADP, and $5 \mu \mathrm{M}$ ADP. With adjustment, high PR was still significantly associated with an increased risk of MACE (Table 3). However, for PR evaluated using $10 \mu \mathrm{M}$ ADP, risk only increased for the highest PR category, corresponding to LTA values greater than $60 \%$.

\section{Interaction between risk level and PR for the outcome of MACE Platelet reactivity assessed with $20 \mu \mathrm{M}$ ADP}

Patients with none of the four risk factors showed no significantly increased risk associated with PR, while for patients with one risk factor only, the higher strata of PR was associated with an increased risk of MACE. Patients with two or more risk factors showed an increased risk of MACE for both the medium and higher strata of PR. (Figure 3). In a Cox model, the interaction between PR strata and the risk level was statistically significant $(p=0.04)$. The corresponding hazard ratios (HRs) are shown in Figure 3. Heterogeneity was not detected for the overall interaction $(p=0.81)$, as well as when it was restricted to each risk level category (intermediate versus low risk level, $p=0.45$, and high versus low risk level, $p=0.90$ ). Additional results on heterogeneity are provided in the supplemental material. Figure 4A shows that PR, when analysed in a continuous fashion, barely affects the risk of MACE at 6 months in patients with no risk factors: the risk is close to $2 \%$ at six months, irrespective of the level of platelet reactivity. Conversely, patients with one risk factor and an overall $4.1 \%$ risk of MACE at six months have in fact a $2 \%$ risk of MACE when they have a low PR, or a 
$6 \%$ risk of MACE when they have a high PR (Figure 4B). Similarly, patients with two or more risk factors and an overall $6 \%$ risk of MACE at six months can indeed have a $2 \%$ risk of MACE when they have a low PR (Figure 4C). The reclassification of the 6-month risk of MACE, according to the three categories of platelet reactivity, in patients with no, one and two or more risk factors, is shown in Table 4. Overall, PR allowed the reclassification of $44 \%$ of the total population (1837/4193 patients) included in a 6-month follow-up to a different level, mostly in patients originally identified as intermediate or high risk on the basis of the number of risk factors only. In patients experiencing MACE in the first 6 months of follow-up, the risk predicted by the combination of PR and risk factors was on average increased compared with the risk predicted from risk factors only: the continuous event net reclassification index $(\mathrm{NRI})$ was $0.39(95 \% \mathrm{Cl} 0.23$ to 0.62$)$. Conversely, in patients free of MACE at 6 months, the measure of PR did not modify the predicted risk: the continuous nonevent NRI was $0.01(95 \% \mathrm{Cl}-0.16$ to 0.09$)$. The overall NRI was $0.39(95 \% \mathrm{Cl} 0.22$ to 0.57$)$.

\section{Platelet reactivity assessed with $10 \mu \mathrm{M}$ ADP}

A total of only five low-risk patients in four studies performing $10 \mu \mathrm{M}$ ADP LTA to assess PR precluded an analysis of this low-risk group. Furthermore, the surrogate for risk level failed to demonstrate an association with the observed risk of MACE in these studies. Figure 4B shows that the risk of MACE increased in both intermediate- and high-risk patients for PR values above $40 \%$, without any obvious relation with the level of risk.

\section{Platelet reactivity assessed with $5 \mu \mathrm{M}$ ADP}

The direction of interaction between PR using $5 \mu \mathrm{M}$ ADP and the risk level was similar to that observed for PR using $20 \mu \mathrm{M}$ ADP, even though overall interaction did not reach the significance level $(p=0.17)$. Of note there were 980 fewer patients in the studies performing 5 $\mu \mathrm{M}$ ADP than in those using $20 \mu \mathrm{M}$ ADP. The increased risk of MACE as PR increases is indeed similar for intermediate- and high-risk patients; for low-risk patients PR is not associated with a MACE outcome (online data supplement). Heterogeneity was not detected 
for the overall interaction $(p=0.19)$. Figure $4 C$ shows that the risk of MACE was unaffected by PR in low-risk patients while it increased for PR values above $30 \%$ in intermediate-risk patients and for PR values above $10 \%-20 \%$ in high-risk patients.

\section{Sensitivity analyses}

Sensitivity analyses were performed for PR using $20 \mu \mathrm{M}$ ADP to assess: the robustness of the association between PR and risk of MACE and its interaction with the level of cardiovascular risk; the robustness of the results in the population of $\mathrm{PCl}$ patients and when target vessel revascularisation is added to the composite outcome. All analyses showed that the sizes of the effects remained similar, and whilst in some instances the statistical significance of the interactions could be lost, there was no impact on their magnitudes (supplemental Tables 1 and 2). Notably, when PR was categorised in quartiles (20 $\mu \mathrm{M}$ ADP maximal aggregation quartiles $=0 \%-38.1 \%, 38.2 \%-51.3 \% .51 .4 \%-63.0 \%, 63.1 \%-100 \%)$ the interaction between PR and the number of risk factors remained significant $(p=0.01)$. When restricted to the population of 3,564 patients treated with $\mathrm{PCl}$ and tested using $20 \mu \mathrm{M}$ ADP the interaction was of similar magnitude but no longer significant (supplemental Table3).

\section{Publication and availability biases}

A check for potential publication bias was made for PR using $20 \mu \mathrm{M}$ ADP, on which the main analyses were performed. A funnel plot was obtained by representing the HR of PR using 20 $\mu \mathrm{M}$ ADP and the standard error, assessed in each separate study (supplemental Figure 4). Two studies with a negative association between PR using $20 \mu \mathrm{M}$ ADP and the risk of MACE (with small sample sizes) were detected as missing using the 'trim and fill' method for making the funnel plot symmetrical. When these missing studies were added, the pooled HR was not significantly modified. These findings suggested that the publication bias in our meta-analysis was minor.

Seven qualifying studies could not provide individual patient data. It is of note that in five of these, the relation between clopidogrel non-response and ischemic events was not a study 
objective (pharmacokinetic-pharmacodynamic studies or randomised trials of different clopidogrel loading doses). The two remaining studies ( $n=101$ and 111 patients) were specifically interested in the prognostic value of PR for MACE.

6

7

8

9

10

11

12

13

14

15

16

17

18

19

20

21

22

23

24

25

26

27

28

29

30

31

32

33

34

35

36

37

38

39

40

41

42

43

44

45

46

47

48

49

50

51

52

53

54

55

56

57

58

59

60

https://mc.manuscrîptcentral.com/bmj 


\section{Discussion}

In the present meta-analysis of individual patient data conducted in clopidogrel-treated patients we demonstrated that the association between PR and the risk of MACE depended strongly on the level of cardiovascular risk. When using $20 \mu \mathrm{M}$ ADP, the most commonly used concentration in LTA, the risk of MACE associated with PR increased with the level of cardiovascular risk. Indeed, PR did not affect the risk of MACE in patients presenting no risk factors, however it gradually increased the risk of MACE as the number of cardiovascular risk factors increased, reaching a 3.7 times greater risk in high-risk patients with a high PR. The measure of PR with $20 \mu \mathrm{M}$ ADP, in addition to risk factors, modified the interpretation of the 6-month risk of MACE in $44 \%$ of patients, mainly in patients with at least one risk factor. Interestingly, smoking and hypercholesterolemia were not associated with the outcome of MACE and were not included in the analysis of the interaction between PR and risk factors. In randomised controlled trials, the benefit of clopidogrel in reducing the incidence of MACE is primarily seen in smokers, with little benefit to non-smokers. ${ }^{39}$ With regard to the cohort studies of clopidogrel-treated patients included in this meta-analysis, this differential effect suggests that the increased risk of MACE related to smoking is offset by the benefit clopidogrel provides to smokers; it thereby weakens any possible analysis of the interaction between smoking and PR for outcomes of MACE. Regarding hypercholesterolemia, this conventional risk factor is likely to be confounded by indications for statin treatment. Indeed, in the ADAPT-DES registry ${ }^{16}$ hyperlipidemia was protective against mortality with a $H R=0.60$ $(0.41-0.86)$ and was not prognostic of MACE in post-ACS patients with optimal medical therapy. ${ }^{40}$ In addition, hypercholesterolemia was not homogeneously defined across the studies in the present meta-analysis and other markers, such as plasma LDL-cholesterol levels, were not widely available.

When PR was evaluated using $5 \mu \mathrm{M}$ ADP, its interaction with the level of cardiovascular risk for the prediction of MACE was of a similar magnitude, although non-significant. These findings may reflect the lower number of patients available in studies using $5 \mu \mathrm{M} A D P$, and a 
corresponding loss of power. Moreover, it was previously shown that ADP-induced platelet aggregation in citrated plasma was dependent on the artifactual generation of TxA2 that was modulated by aspirin, at least at lower ADP concentrations. ${ }^{41}$ This may be associated with an additional background noise in which the interaction between the identified risk factors and PR to predict MACE is blurred, as seen with the lowest concentrations of ADP. Only four of the studies analysed used $10 \mu \mathrm{M}$ ADP, and two of these had a follow-up limited to 30 days; with only 124 MACEs during follow-up, this accounts for a limitation in power to reliably study interactions. Which laboratory assay and which platelet agonist concentration are best suited for the clinical evaluation of platelet function is the matter of some debate. ADP-induced LTA is highly reproducible within a given laboratory, but its lack of standardisation across studies may have slightly weakened the positive findings or lower the level of significance for the interactions found in the present meta-analysis. Of note, the present meta-analysis does not aim to promote the use of LTA to tailor antiplatelet therapy but it rather relied on a historical gold standard in platelet function testing to evidence an interaction with patients'characteristics that should be considered for a tailored approach. The point-of care VerifyNow $\mathrm{P}_{2} \mathrm{Y}_{12}$ assay, used in several intervention trials, correlates well with ADP-induced LTA $^{4243}$ and we speculate that the main findings of the present meta-analysis would have been similar, had PR been evaluated using the VerifyNow $\mathrm{P}_{2} \mathrm{Y}_{12}$ assay.

Several intervention trials have compared conventional clopidogrel treatment to an antiplatelet strategy tailored according to PR. Early, small randomised trials ${ }^{1112}$ that utilised vasodilator-stimulated phosphoprotein phosphorylation level measurement to indicate $\mathrm{P}_{2} \mathrm{Y}_{12}$ receptor reactivity, showed a protective effect for repeat $600 \mathrm{mg}$ clopidogrel loading doses in ACS patients prior to $\mathrm{PCl}$. However, recent larger trials utilising the VerifyNow $\mathrm{P}_{2} \mathrm{Y}_{12}$ assay were negative. Indeed, the GRAVITAS ${ }^{13}$ and ARCTIC $^{14}$ studies failed to show the benefit of a PR-tailored antiplatelet strategy after PCI. Various limitations of these trials were addressed in a recent consensus publication. ${ }^{22}$ The event rate of the GRAVITAS study was low compared to the one used for power calculation, and the antiplatelet effect of the high-dose regimen may have been suboptimal as it reduced the prevalence of high PR by only $22 \%$. 
Similarly, the ARTIC study population was also at a low absolute risk of subsequent cardiovascular events because the prevalence of ACS patients was low, and the composite endpoint also included other events that may not be related to platelet function. The interaction of PR and the number of risk factors, as identified in the present meta-analysis, substantiates the hypothesis that the risk associated with high PR was not clinically relevant in low-risk patients, and that any measure aiming to lower PR is unlikely to lead to a beneficial reduction of MACE for these low-risk patients. Based on these observations we speculate that higher risk patients are more likely to benefit from a therapy tailored to their initial PR. This may explain why early interventions designed to efficiently blunt high PR in ACS patients with multiple conventional risk factors translated into a reduction of MACE. ${ }^{11} 12$ 22.

In the current new antiplatelet era, prasugrel and ticagrelor have a major part to play in the management of ACS, leaving clopidogrel as an alternative for patients with high bleeding risk. However, a recent cost-effectiveness analysis for six European perspectives showed that the universal use of newer $\mathrm{P}_{2} \mathrm{Y}_{12}$ inhibitors for ACS patients is probably not as cost-effective as strategies based on PR. ${ }^{44}$ It should also be kept in mind that ticagrelor and prasugrel increase the risk of bleeding and that a therapeutic medium-PR window is associated with optimal net clinical benefit. ${ }^{22}$ The net benefits of newer $\mathrm{P}_{2} \mathrm{Y}_{12}$ inhibitors could also probably be improved not only by testing for PR, but also by incorporating patient risk levels in the decision-making process. Although ongoing trials on tailored $\mathrm{P}_{2} \mathrm{Y}_{12}$ strategies, including TROPICAL-ACS (ClinicalTrials.gov identifier: NCT01959451) and ANTARCTIC ${ }^{45}$ partly include this concept of risk levels, further efforts in this direction are needed. This meta-analysis has several strengths, such as the good overall quality of the studies included, as assessed using a quality tool specifically adapted to prognostic studies. The availability of individual patient data allowed a reliable evaluation of the risk associated with PR and of the interaction with vascular risk factors. Readily available risk factors relevant to a secondary prevention population were thus identified. The consistency of results across the 
different ADP concentrations used in the different studies to assess PR, as well as the sensitivity analyses, indicated that the results were robust.

Despite the advantages related to the availability of individual patient data, this meta-analysis also had some limitations, including a low proportion of women (25\%). This did not allow a stratification of the analyses by gender, as is usually the case in risk assessment tools such the European SCORE or the Framingham risk score. Indeed, in these latter scores gender is not considered as one of traditional risk factors, but is rather presented in separate charts for women and men. There were incomplete data on concomitant medications or other relevant risk factors such as the left ventricular ejection fraction, cholesterol levels or renal insufficiency. Finally, information on bleeding was limited to five studies and a low number of events, thus precluding a reliable analysis of bleeding events and their relation to PR. In conclusion, high PR in patients on clopidogrel is associated with an increased risk of MACE in patients with vascular risk factors, but not in low-risk patients. These findings suggest that trials on tailored PR treatment strategies should be primarily stratified on the individual vascular risk factors in order to assess a truly personalized approach.

Funding: No external funding. Conducted within the Geneva Platelet Group and the division of Clinical Epidemiology.

\section{Acknowledgments:}

We thank P Whiting for advices on the evaluation of the quality using the PROBAST tool: The PROBAST tool for the assessment of risk of bias and concerns about applicability for prognostic studies was kindly provided by the PROBAST steering group. This tool is still in development and is available from the PROBAST team (www.systematic-reviews.com/probast).

\section{Contributors:}

Reny JL, Fontana $\mathrm{P}$, and Combescure $\mathrm{C}$ are guarantors for the study, had full access to the data and take responsibility for the integrity of the data and the accuracy of its analysis 
Study concept and design: Reny JL, Fontana P, and Combescure C

Acquisition of data: Reny JL, Fontana P, Hochholzer W, Neumann FJ, Ten Berg J, Janssen

PW, Geisler T, Gawaz M, Marcucci R, Gori AM, Cuisset T, Alessi MC, Berdagué P, Gurbel P, Yong G, Angiolillo D, Aradi D, Beigel R, Campo G.

Data management and statistical analysis: Combescure C and Reny JL

Drafting and critical revision of the manuscript for important intellectual content:: Reny JL, Fontana P, Hochholzer W, Neumann FJ, Ten Berg J, Janssen P, Geisler T, Gawaz M, Marcucci R, Gori AM, Cuisset T, Alessi MC, Berdagué P, Gurbel P, Yong G, Angiolillo D, Aradi D, Beigel R, Campo G, Combescure C.

All authors had full access to all of the data (including statistical reports and tables) in the study and can take responsibility for the integrity of the data.

\section{Conflicts of interest:}

Reny JL: payment for lectures by Merck Sharp and Dohme

Fontana P: consultancy for Evolva; grants from Evolva and Astra Zeneca, payment for lectures by Bayer, AstraZeneca.

Hochholzer W: no conflicts of interest.

Neumann FJ: no conflicts of interest

Ten Berg J: consultancy for Astra Zeneca, Eli Lilly, Merck, and Daiichi Sankyo Janssen P: no conflicts of interest

Geisler T: consultancy for Bayer, Medicines company, Eli Lilly, Pfizer, BMS, and Daiichi Sankyo; payments for lectures by Bayer, Medicines company, Eli Lilly, Pfizer, BMS, and Daiichi Sankyo; MSD, Boehringer, Astra Zeneca.

Gawaz M: consulting fee for Bayer, Astra Zeneca, MSD. Lilly; consultancy for BoehringerIngelheim

Marcucci R: no conflicts of interest

Gori AM: no conflicts of interest

Cuisset T: no conflicts of interest 
Alessi MC: Board membership for Astra Zeneca and Lilly; lectures for Roche;

Berdagué P: no conflicts of interest

Gurbel P: Served as a consultant for Daiichi Sankyo, Sankyo, Lilly, Bayer, AstraZeneca, Accumetrics, Merck, Medtronic, CSL, and Haemonetics; receiving grants from the National Institutes of Health, Daiichi Sankyo, Lilly, CSL, AstraZeneca, Harvard Clinical Research Institute, Haemonetics, and Duke Clinical Research Institute; receiving payment for lectures, including service on speakers' bureaus, from Lilly, Daiichi Sankyo, and Merck; receiving payment for development of educational presentations from Merck, the Discovery Channel, and Pri-Med; Dr. Gurbel holds stock or stock options in Merck, Medtronic, and Pfizer; and holds patents in the area of personalised antiplatelet therapy and interventional cardiology. Yong G: no conflicts of interest Angiolillo DJ: Received payment as an individual for: a) Consulting fee or honorarium from Bristol-Myers Squibb, Sanofi-Aventis, Eli Lilly, Daiichi Sankyo, Inc., The Medicines Company, AstraZeneca, Merck, Evolva, Abbott Vascular, and PLx Pharma; b) Participation in review activities from Johnson \& Johnson, St. Jude, and Sunovion ; c) has received institutional payments for grants from Bristol-Myers Squibb, Sanofi-Aventis, GlaxoSmith Kline, Otsuka, Eli Lilly, Daiichi Sankyo, Inc., The Medicines Company, AstraZeneca, Evolva, Gilead; and has other financial relationships with Esther and King Biomedical Research Grant. Aradi D: consultancy for Verum Diagnostica $\mathrm{GmbH}$; lectures for Verum Diagnostica, Roche, DSI/Lilly, Bayer, Astra-Zeneca, Pfizer, Biotronic, Abbott.

Beigel R: no conflicts of interest

Campo G: no conflicts of interest

Combescure C: no conflicts of interest 


\section{What this paper adds}

What is already known on this subject

Prior meta-analyses have shown an association between high on-clopidogrel platelet reactivity (PR) and the risk of major adverse cardiovascular events (MACE). However, data are heterogeneous and large intervention trials on PR-tailored treatments have been neutral, possibly owing to the inclusion of patients at low cardiovascular risk. The role and usefulness of PR with regard to levels of cardiovascular risk are unclear and may explain these discrepancies.

What this study adds

- The magnitude of the association between PR and MACE risk is strongly dependant on the level of cardiovascular risk faced by patients suggesting that trials on tailored PR treatment strategies should be primarily stratified on the individual vascular risk and clinical setting.

- This study suggests that medical policies edicted around the concept of personalized medicine should not be restricted to a single biological phenotype or single nucleotide variant but should also emphasize the role of individual clinical risk factors. 
Table 1. Main characteristics of published studies

\begin{tabular}{|c|c|c|c|c|c|c|c|c|c|c|c|c|c|}
\hline Study & $\begin{array}{l}\text { Years of } \\
\text { publication }\end{array}$ & $\begin{array}{l}\text { Patients } \\
\text { (n) }\end{array}$ & $\begin{array}{c}\text { Age } \\
(y)\end{array}$ & $\begin{array}{c}\text { Male } \\
(\%)\end{array}$ & $\begin{array}{c}\text { Diabetics } \\
(\%)\end{array}$ & $\begin{array}{c}\text { Smokers } \\
(\%)\end{array}$ & $\begin{array}{l}\text { Hyper- } \\
\text { tension } \\
(\%)\end{array}$ & $\begin{array}{c}\text { Hypercholes- } \\
\text { terolemia } \\
(\%)\end{array}$ & $\begin{array}{c}\text { ACS at } \\
\text { inclusion } \\
(\%)\end{array}$ & $\begin{array}{l}\mathrm{PCl} \\
(\%)\end{array}$ & $\begin{array}{c}\text { Gpllb/llla } \\
\text { inhibitor } \\
(\%)\end{array}$ & $\begin{array}{l}\text { Follow-up } \\
\text { (months) }^{*}\end{array}$ & $\begin{array}{l}\text { ADP } \\
(\mu \mathrm{M})\end{array}$ \\
\hline Campo et al. ${ }^{2 \prime}$ & 2006 & 70 & $64 \pm 13$ & 69 & 19 & 37 & 63 & 34 & 100 & 100 & 100 & $10(15)$ & 5,20 \\
\hline Hochholzer et al. ${ }^{28}$ & 2006 & 765 & $66 \pm 9$ & 78 & 24 & 11 & 82 & 92 & 0 & 100 & 0 & $12(12)$ & 5,20 \\
\hline Cuisset et al. ${ }^{30}$ & 2007 & 190 & $65 \pm 12$ & 76 & 33 & 48 & 58 & 53 & 87.4 & 100 & 14.7 & $1(1)$ & 10,20 \\
\hline Geisler et al. ${ }^{31}$ & 2008 & 1,092 & $67 \pm 11$ & 74 & 33 & 39 & 80 & 59 & 51.7 & 100 & 7.7 & $1(1)$ & 20 \\
\hline Cuisset et al. ${ }^{33}$ & 2009 & 598 & $65 \pm 12$ & 78 & 35 & 39 & 56 & 55 & 100 & 100 & 9.9 & $1(1)$ & 10 \\
\hline Yong et al. ${ }^{34}$ & 2009 & 248 & $63 \pm 12$ & 71 & 22 & 27 & 53 & 52 & 100 & 55 & 39.7 & $6(21)$ & $5,10,20$ \\
\hline Breet et al. ${ }^{35}$ & 2010 & 1,069 & $64 \pm 11$ & 75 & 81 & 11 & 77 & 80 & 0 & 100 & 7.0 & $12(12)$ & 5,20 \\
\hline Marcucci et al. ${ }^{36}$ & 2010 & 1,108 & $69 \pm 10$ & 75 & 24 & 23 & 66 & 55 & 100 & 100 & 26.0 & $12(12)$ & 10 \\
\hline Beigel et al. ${ }^{3 /}$ & 2011 & 174 & $59 \pm 12$ & 83 & 27 & 41 & 51 & 45 & 100 & 100 & - & $6(6)$ & 5 \\
\hline
\end{tabular}

Age, mean \pm standard deviation; CAD, coronary artery disease; ACS, acute coronary syndrome; PCl, percutaneous coronary intervention; ADP, adenosine diphospate concentration used for the evaluation of platelet reactivity

* Median (maximum) 
Table 2: Multivariate analysis to assess the associations between the risk factors and the composite outcome of MACE. This analysis was conducted on the patients of the 13 studies of the meta-analysis ( $n=6,256$ after exclusion of missing data). MACE were observed in 412 patients. Hazard ratios (HR) greater than one show an increased risk of MACE in patients having the corresponding risk factor.

\begin{tabular}{|c|c|c|c|c|c|}
\hline Factors collected in studies & Adjusted HR [95\% Cl] & $p$ & Level of risk of MACE * & $\mathrm{HR}[95 \% \mathrm{Cl}]$ & p \\
\hline Current smoking status & $0.92[0.71 ; 1.18]$ & 0.50 & Low risk ( $n=579$ ) & 1 & \\
\hline Age (> 75) & $1.56[1.25 ; 1.95]$ & $<0.0001$ & Intermediate risk ( $n=2444)$ & $1.61[1.05 ; 2.45]$ & 0.03 \\
\hline Diabetes & $1.58[1.27 ; 1.96]$ & $<0.0001$ & High risk ( $n=3435)$ & $2.58[1.69 ; 3.94]$ & $<0.0001$ \\
\hline Hypercholesterolemia & $0.86[0.69 ; 1.06]$ & 0.15 & & & \\
\hline Hypertension & $1.23[0.98 ; 1.54]$ & 0.07 & & & \\
\hline ACS at inclusion & $2.00[1.27 ; 3.16]$ & 0.003 & & & \\
\hline Gender (Male) & $1.11[0.89 ; 1.40]$ & 0.35 & & & \\
\hline
\end{tabular}

*: a surrogate for the level of risk was defined as the number of risk factors (among age, diabetes, hypertension, and ACS at inclusion): low risk for no risk factor, intermediate risk for one risk factor and high risk for two or more risk factors). 
Table 3: Associations between the ADP categories and the composite outcome of MACE with adjustment on the factors collected in the studies of the meta-analysis (factors shown in Table 2).

\begin{tabular}{|c|c|c|c|c|c|c|}
\hline & \multirow{2}{*}{\multicolumn{2}{|c|}{ ADP $20 \mu \mathrm{M}$}} & \multirow{2}{*}{\multicolumn{2}{|c|}{ ADP $10 \mu \mathrm{M}$}} & \multirow{2}{*}{\multicolumn{2}{|c|}{ ADP $5 \mu \mathrm{M}$}} \\
\hline & & & & & & \\
\hline & $\mathbf{N}$ & & $\mathbf{N}$ & & $\mathbf{N}$ & \\
\hline Studies & 9 & & 4 & & 8 & \\
\hline Events & 287 & & 124 & & 229 & \\
\hline \multirow[t]{2}{*}{ Patients (after exclusion of missing data) } & 4,140 & & 2,077 & & 3,160 & \\
\hline & $\mathrm{HR}[95 \% \mathrm{Cl}]$ & $\mathbf{p}$ & $\mathrm{HR}[95 \% \mathrm{Cl}]$ & $\mathbf{p}$ & $\mathrm{HR}[95 \% \mathrm{Cl}]$ & $\mathbf{p}$ \\
\hline ADP & & 0.0003 & & 0.03 & & 0.02 \\
\hline Lower category * & 1 & & 1 & & 1 & \\
\hline Intermediate category * & $1.85[1.26 ; 2.73]$ & 0.002 & $1.31[0.79 ; 2.17]$ & 0.30 & $1.79[1.02 ; 3.14]$ & 0.04 \\
\hline Higher category * & $2.91[1.78 ; 4.74]$ & $<0.0001$ & $2.61[1.64 ; 4.16]$ & $<0.0001$ & $2.79[1.50 ; 5.22]$ & 0.001 \\
\hline \multicolumn{7}{|c|}{ * Categories for ADP 20 and $10 \mu \mathrm{M}$ are $0 \%-40 \%, 41 \%-60 \%, 61 \%-100 \%$, } \\
\hline \multicolumn{7}{|c|}{ and for ADP $5 \mu \mathrm{M}$ are 0\%-30\%, 31\%-50\%, 51\%-100\% } \\
\hline
\end{tabular}


Table 4: Reclassification of the 6-month risk of MACE when the individual risk was predicted from platelet reactivity measured by $20 \mu \mathrm{M}$ ADP in addition to risk factors. The predicted risk was stratified in three levels (low: $\leq 3 \%$, intermediate: $>3 \%$ and $\leq 5 \%$, high: $>5 \%$ ) in agreement with the 6 -month risk observed in patients with none, one and two or more risk factors $(2.3 \%, 4.1 \%$ and $6.2 \%$ respectively). Patients were stratified according to their number of risk factors and to the level of the predicted risk. The numbers of patients and, in brackets, the corresponding observed 6-month risk of MACE in each stratum.

Risk predicted by the combination of risk factors and platelet

reactivity measured by $20 \mu \mathrm{M}$ ADP

\begin{tabular}{|c|c|c|c|c|c|}
\hline & & & & & \multirow[b]{2}{*}{ Total } \\
\hline & & $\begin{array}{l}\text { Low risk } \\
\text { ( } \leq 3 \%)\end{array}$ & $\begin{array}{l}\text { Intermediate risk } \\
\text { (>3\% and } \leq 5 \%)\end{array}$ & $\begin{array}{c}\text { High risk } \\
(>5 \%)\end{array}$ & \\
\hline Risk predicted by & Low risk - no risk factor & $524^{*}(2.4 \%$ **) & 26 * & 0 * & $550 *\left(2.3 \%{ }^{* *}\right)$ \\
\hline the number of risk & Intermediate risk - one risk factor & $625 *(2.1 \% * *)$ & $576 *(3.7 \% * *)$ & $622 *(6.3 \% * *)$ & $1823 *(4.1 \% * *)$ \\
\hline \multirow[t]{2}{*}{ factors only } & High risk - two or more risk factors & $102 *(0.0 \% * *)$ & $462 *(3.0 \% * *)$ & $1256 *(7.6 \% * *)$ & $1820 *(6.2 \% * *)$ \\
\hline & Total & $1251^{*}(2.1 \% * *)$ & $1064 *(3.4 \% * *)$ & $1878 *(7.1 \%$ ** $)$ & $4193^{*}\left(4.7 \%{ }^{* *}\right)$ \\
\hline
\end{tabular}




\section{Figure Legends}

\section{Figure 1: Flow chart of the meta-analysis}

Figure 2: Kaplan-Meier survival curve for the occurrence of MACE

Figure 3: Association between platelet reactivity and the occurrence of MACE according to the level of risk

Low-risk patients have none of the risk factors (among age > 75 years, acute coronary syndrome at inclusion, diabetes, and hypertension), intermediate-risk patients have one risk factor and high-risk patients have two or more risk factors. PR was assessed with $20 \mu \mathrm{M}$ ADP LTA.

Figure 4: 6-month risk of MACE according to platelet reactivity in the different risk groups. The dashed line represents the overall risk, ignoring platelet reactivity and the black line shows the risk according to the platelet reactivity assessed with $20 \mu M$ ADP LTA, in patients with no risk factors $(A)$, one risk factor $(B)$ and two or more risk factors $(C)$. 
Figure 1

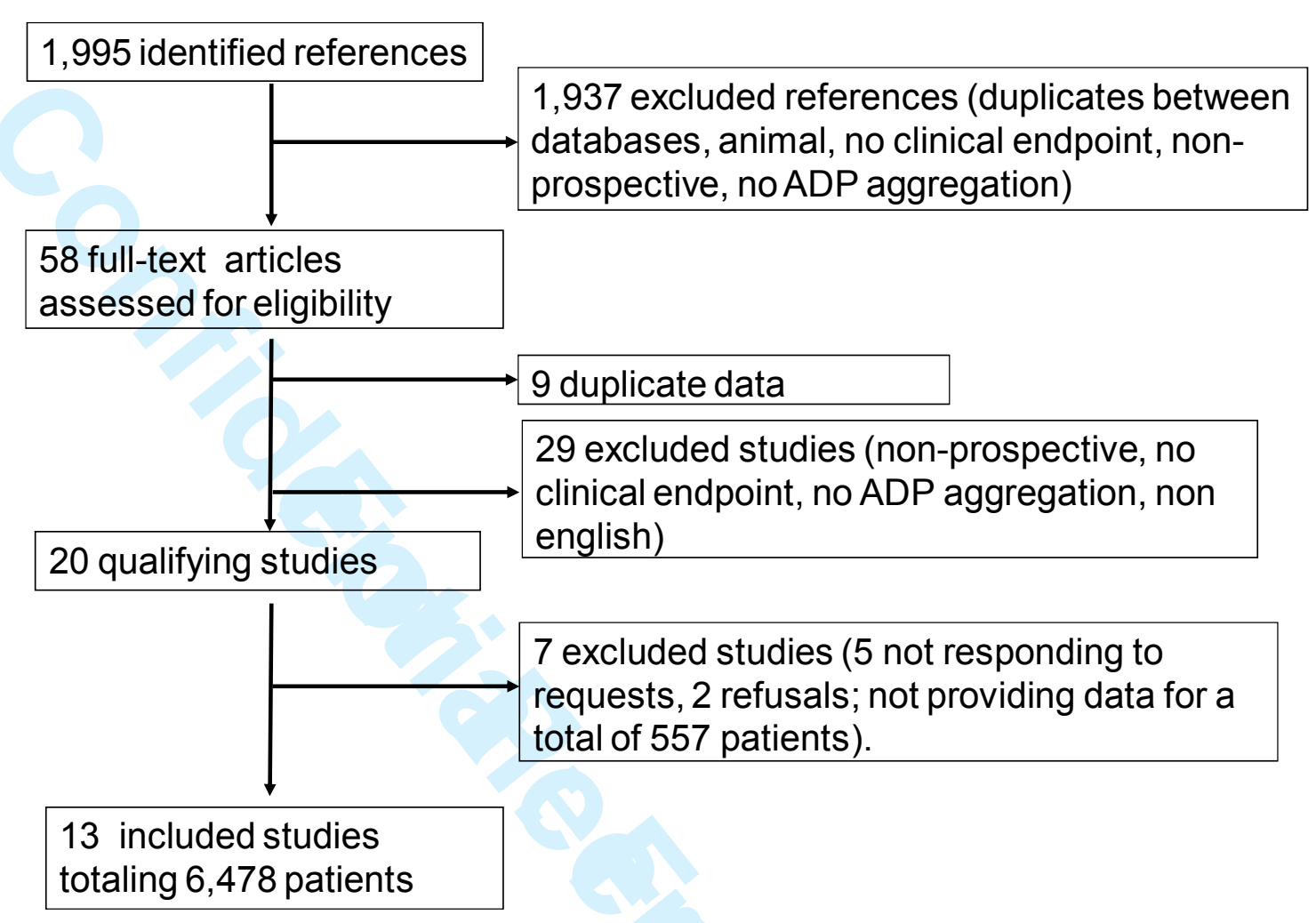




\section{Figure 2}
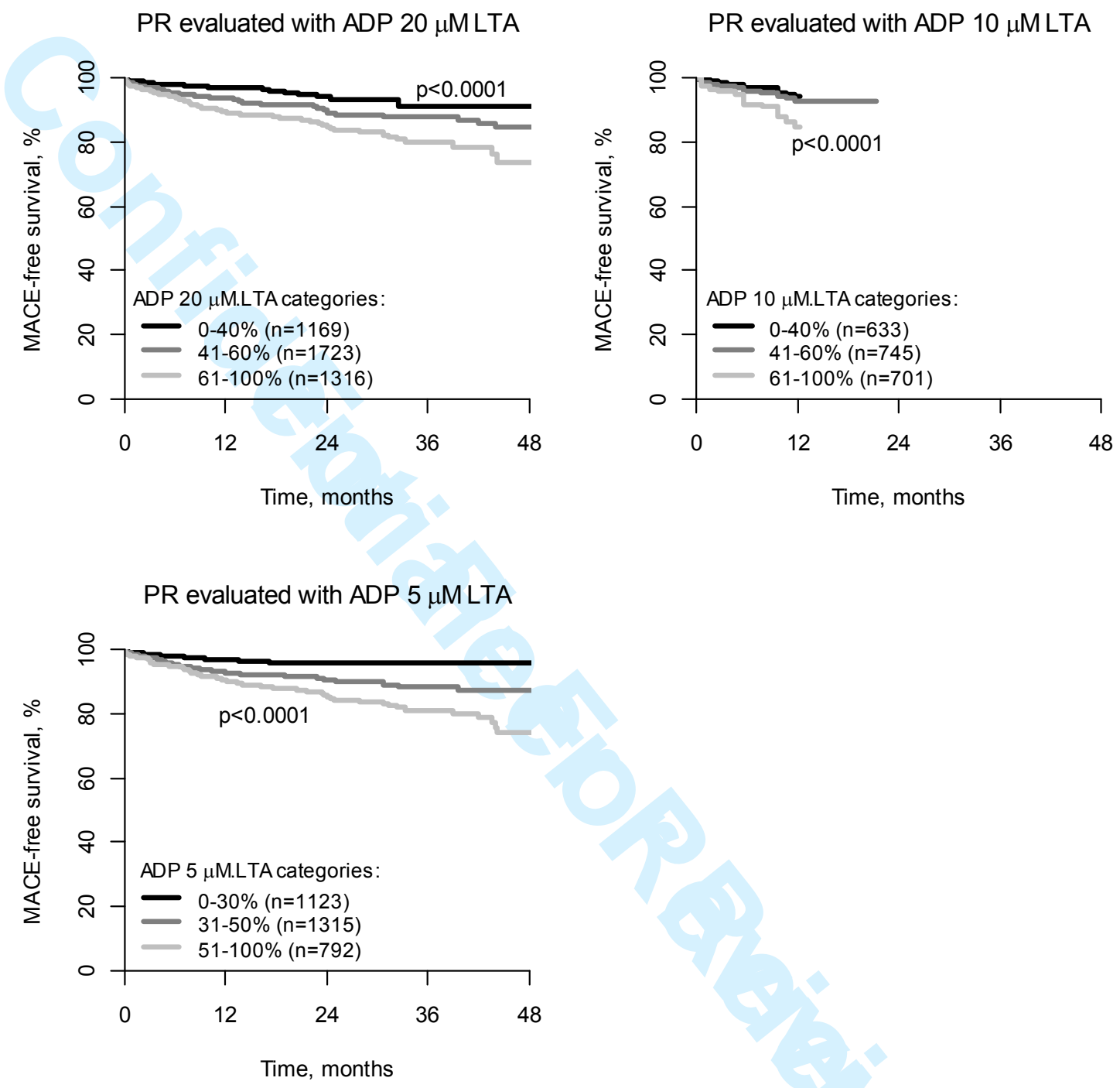

https://mc.manuscriptcentral.com/bmj 


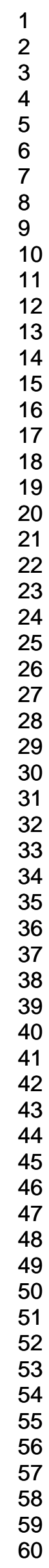

https://mc.manuscriptcentral.com/bmj 


\section{Figure 3}
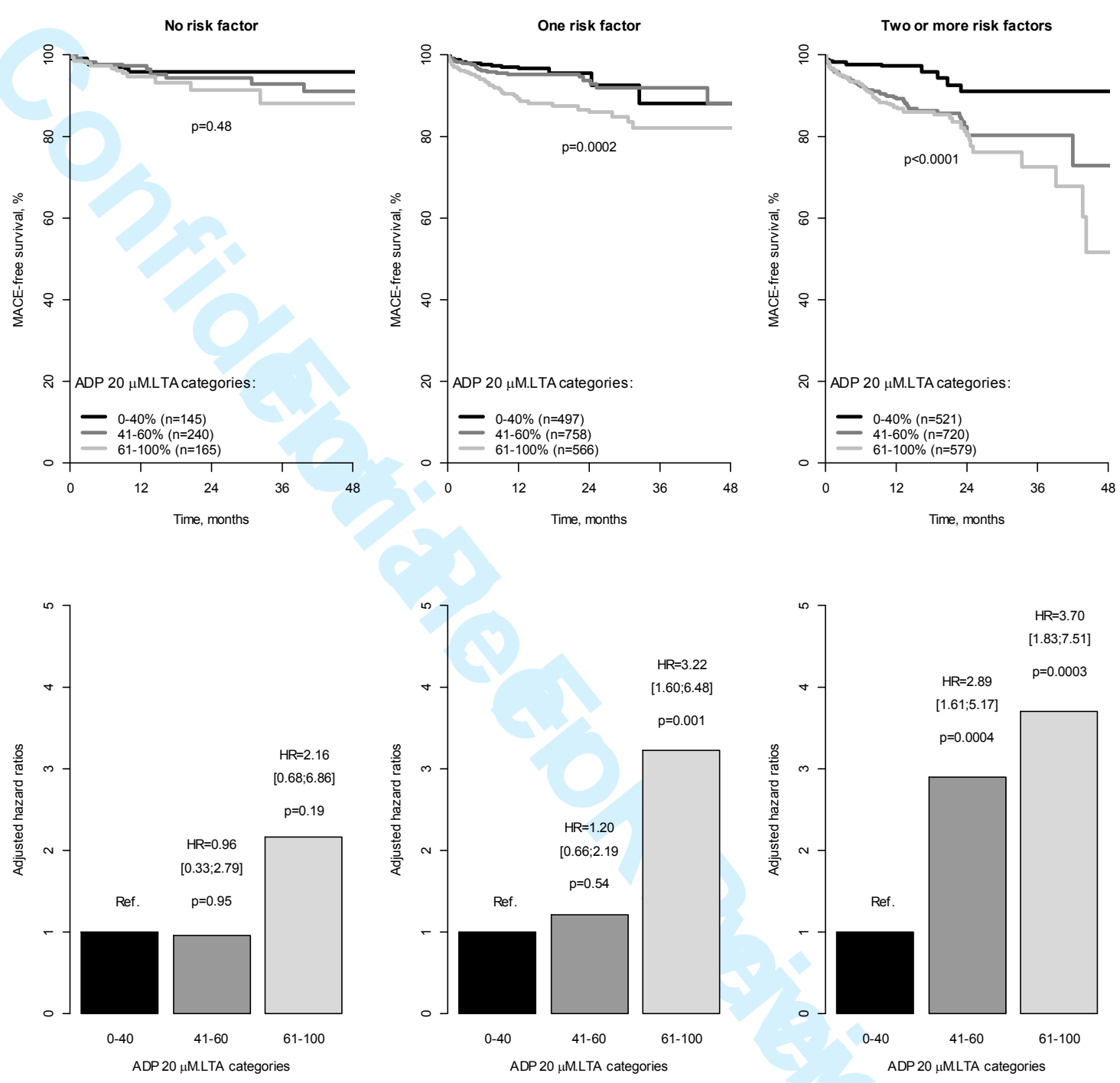

41

42

43

44

45

46

47

48

49

50

51

52

53

54

55

56 


\section{Figure 4}
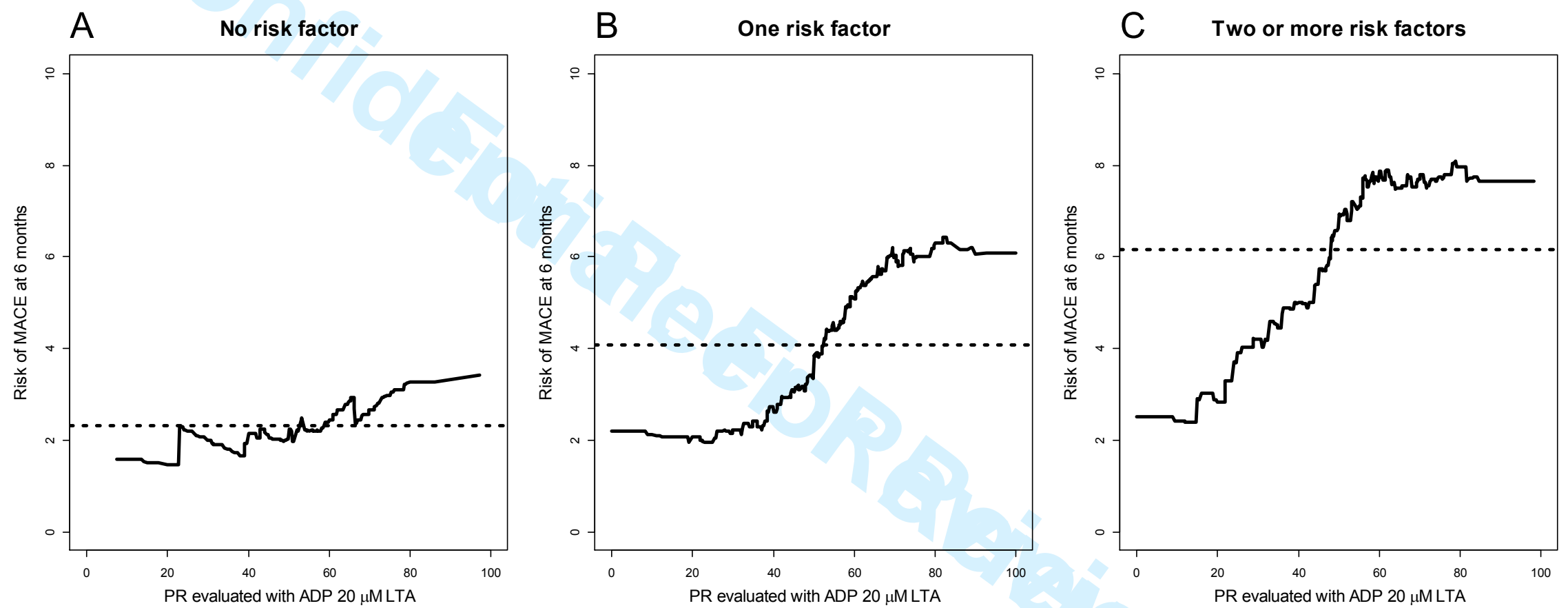


\section{Supplemental material}

Vascular risk levels affect the predictive value of platelet reactivity for the occurrence of major adverse cardiovascular events in patients on clopidogrel:

Systematic review and collaborative meta-analysis of individual patient data

\section{Content}

Detailed statistical analysis

Complementary characteristics of studies, risks of bias, and concerns regarding applicability

Supplemental Figure 1. Criteria to assess risks of bias and concerns about applicability

Supplemental Figure 2. Result of the assessment by domains for risk of bias and concerns about applicability

Cox models and assumption of proportionality of hazards

Complementary results on heterogeneity

Detailed results on studies using $5 \mu \mathrm{M}$ ADP to assess platelet reactivity

Supplemental Figure 3. Interaction between $5 \mu \mathrm{M}$ ADP LTA values and the level of risk

Sensitivity analyses

Supplemental Table 1. Sensitivity analysis for the association between PR using $20 \mu \mathrm{M}$ ADP and risk of MACE (leave-one-out procedure)

Supplemental Table 2. Sensitivity analysis for the modification of the association between PR, assessed using $20 \mu \mathrm{M}$ ADP and risk of MACE

Supplemental Table 3. Sensitivity analysis in patients with PCI at inclusion and when target vessel revascularisation (TVR) is included in the composite outcome

Supplemental Figure 4. Funnel plot for detection of a potential publication bias 


\section{Detailed statistical analysis}

MACE-free survival curves were obtained using the Kaplan-Meier estimator and by pooling data from studies. Comparisons between subgroups of PR with ADP 20, 10, and $5 \mu \mathrm{M}$ were performed using log-rank tests stratified on the studies. A surrogate of the individual level of risk of MACE was obtained by identifying the factors associated with the MACE outcome in a multivariate mixed-effect Cox model and by counting the number of these factors. The tested factors were the traditional risk factors (age, hypercholesterolemia, diabetes, hypertension, smoking, and acute ischemic event at inclusion). Other risk factors, such as body mass index or family history, were available in only a limited number of studies and were not included as covariates. The between-study variability in the baseline hazard was accounted for by a random coefficient. This analysis was conducted on the whole sample with the $\mathrm{R}$ package 'coxme'. 4647

In subsets of studies reporting PR evaluated using $20 \mu \mathrm{M}, 10 \mu \mathrm{M}$, and $5 \mu \mathrm{M}$ ADP, associations between $\mathrm{PR}$, expressed in categories (low, intermediate, high PR) and the risk of MACE, was analysed using mixed-effect Cox models with adjustment for traditional risk factors. The between-study variability was accounted for by a random coefficient for the baseline hazard and for each category of PR. The surrogate for the level of MACE risk (number of risk factors) was explored as a modifier of the association between PR and the risk of MACE: the interaction term was tested in a mixed-effect Cox model. The HRs were reported for intermediate and high PR categories, taking the category low as the reference and according it to the number of risk factors. To better describe the modification of the associations between PR and the risk of MACE, the MACE-free survival rates in patients at low-, intermediate-, and high-risk were assessed according to $\mathrm{PR}$, as continuous variables. This analysis was conducted using the $\mathrm{R}$ package prodlim, using the symmetrical nearest neighbourhoods method ${ }^{24}$. The assumption of the proportionality of hazards was tested for all models using Cox models, ${ }^{48}$ since this procedure was not available for mixed-effect Cox models and by plotting the complementary log-log survival against the logarithm of time. Sensitivity analyses were conducted to check the robustness of the findings with respect to the risks of bias and concerns for applicability of studies, as well as the definition of MACE including target vessel revascularisation and the influence of a given specific study (leaveone-out analysis). A potential publication bias was visually inspected on a funnel plot. The 'trim and fill' method was also applied to detect missing studies (for the funnel plot to be symmetric) and to test the sensitivity of the estimate to these missing studies. ${ }^{49}$ The improvement in the assessment of 6-month risk of MACE related to the measure of platelet 
reactivity was evaluated by using the net reclassification index for survival data [REF PENCINA].The event and non-event continuous NRIs were reported. When the continuous event NRI is positive, the predicted risk in patients experiencing MACE is more often increased than decreased, following the addition of PR to risk factors than when it involves risk factors only. Similarly, when the continuous non-event NRI, the predicted risk in patients experiencing MACE is more often decreased than increased when the prediction involves PR in addition to risk factors than when it involves risk factors only. To assess the event and non-event NRIs, we used the risk assessed according to PR as a continuous variable (previously described).

All analyses were conducted using $\mathrm{R}$ version 3.0.1 (R Development Core Team. R: $A$ Language and Environment for Statistical Computing, Vienna, Austria: R Foundation for Statistical Computing; 2010) and Comprehensive Meta-Analysis Version 2 (Biostat, Engelwood, NJ, USA). P-values less than 0.05 were considered significant and all tests were two-sided.

\section{Complementary characteristics of studies, risks of bias, and concerns regarding applicability}

In several instances, data on covariates were not available. For example, data on the use of proton pump inhibitors and statins were not available from eight studies. Aspirin was part of the treatment for all patients in ten studies, and was given to $79 \%, 90 \%$, and $95 \%$ of the patients in the three remaining studies. All but one study included coronary artery disease (CAD) patients exclusively; this last study ${ }^{8}$ included $85 \%$ CAD patients, $10 \%$ peripheral arterial disease patients, and $5 \%$ ischemic stroke patients. $\mathrm{PCl}$ was performed on $87 \%$ of patients. The studies differed markedly with respect to the frequency of diabetes $(19 \%-$ $100 \%)$, smoking $(11 \%-55 \%)$, the use of Gpllb/llla inhibitors $(0 \%-100 \%)$, the ADP concentration used for aggregation tests $(5-20 \mu \mathrm{M})$ to assess $\mathrm{PR}$, and the presence of acute ischemia at inclusion (0\%-100\%). The median follow-up was 12 months, and in all the studies the modalities of the follow-up were identical for clopidogrel responders and nonresponders. LTA was most frequently performed using ADP $20 \mu \mathrm{M}$.

Overall risks of bias and concerns about applicability of the studies were low (Figures 1 and 2). In one study using LTA with ADP $10 \mu \mathrm{M}$, MACE was defined as stent thrombosis during a 30-day follow-up period; ${ }^{33}$ however, with no specific information on myocardial infarction or stroke (as was the case in most of the studies), this lead to a potential high risk of outcome bias. In three studies, ${ }^{27} 3638$ the risk of outcome bias was unclear because either they did not include stroke in the composite outcome of MACE or they did not mention whether adjudication of the outcome was performed blinded to PR test results. In another study, using LTA using ADP $20 \mu \mathrm{M}$, the risk of bias with respect to flow and timing was unclear as $13 \%$ of 
patients had been lost to follow-up. ${ }^{31}$ The characteristics, PR test results, and treatments of these patients were similar to the rest of the cohort. Regarding their applicability, only two studies caused concern, because of an exclusively diabetic population in one ${ }^{29}$ and because of the absence of upper and lower limits to the platelet counts of the patients included in the second. $^{38}$ 
Supplemental Figure 1 Criteria to assess risks of bias and concerns about applicability. Derived from the PROBAST tool available at www.systematic-reviews.com/probast

\section{Risk of bias}

\section{Domains}

Patient selection

Predictor ADP LTA and covariates

Outcome

Flow and timing

\section{Concerns about applicability \\ Domains \\ Patient selection \\ Predictors \\ Outcome}

\section{Criteria}

Consecutive

Inappropriate inclusion or exclusion criteria

Disease at similar stage (inclusion)

Pre-specified or standard technique used

ADP LTA done at the same time for all participants

Vascular risk factors available

All items defining MACE available

MACE diagnosed blinded to ADP test results

Adjudicating committee blinded to ADP test results

Same MACE definition for all participants

ADP test results did not form part of MACE outcome

All patients included in the analysis

Lost to follow up

Clopidogrel was not stopped during follow-up

All the patients benefited from the same MACE assessment

Exact date of MACE known

\section{Criteria}

Sample representative of review's target population

Standard definition for covariates

Unexpected relative frequency of one or more MACE items

Differences in the quality of assessment of each MACE item

The time of assessment of MACE is relevant to the clinical situation 
Supplemental Figure 2: Result of the assessment by domains for risks of bias and concerns about applicability

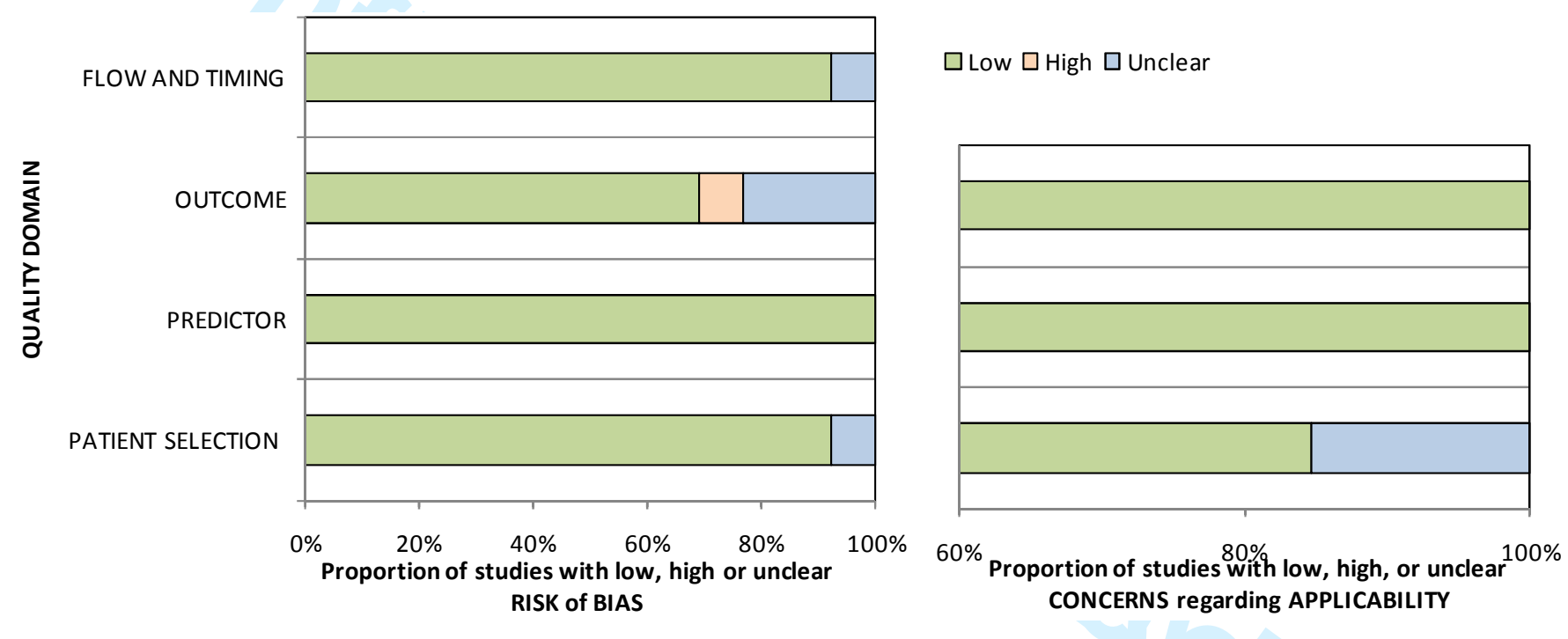




\section{Cox models and assumption of proportionality of hazards}

For the multivariate Cox model stratified on studies and conducted on the whole sample to identify risk factors, the hazards were found to be approximately proportional. The p-value for the test on residuals was greater than 0.10 for any factor. A visual inspection of the log minus log survival plots did not reveal any major deviation from the proportionality of hazards. When PR evaluated using ADP $20 \mu \mathrm{M}$ was added in the model, the p-values were 0.33 for the $41 \%-60 \%$ PR category and 0.28 for the $61 \%-100 \%$ category. When PR evaluated using ADP $10 \mu \mathrm{M}$ was added in the model, the p-values were 0.89 for the $41 \%-60 \%$ PR category and 0.63 for the $61 \%-100 \%$ category. When PR evaluated using ADP $5 \mu \mathrm{M}$ was added in the model, the p-values were 0.72 for the $31 \%-50 \%$ PR category and 0.19 for the $51 \%$ $100 \%$ category. A visual inspection of the log minus log survival plots revealed that when using $10 \mu \mathrm{M}$ ADP, the survival curves crossed in the first 20 days of follow-up. However, the survival in this period was close to one and the cross was not meaningful. In models testing the interaction between PR (evaluated using $20 \mu \mathrm{M}$ ADP and $5 \mu \mathrm{M}$ ADP) and the number of risk factors, $p$-values for all coefficients were greater than 0.20 .

\section{Complementary results on heterogeneity}

The amount of heterogeneity is represented by the variance of the random effects ${ }^{23}$ corresponding to the between-study variance $\left(\mathrm{Tau}^{2}\right)$. Theses variances are reported in the following table for the mixed-effects Cox model when PR is evaluated using ADP $20 \mu \mathrm{M}$.

\begin{tabular}{lc}
\hline Random effects & $\mathrm{Tau}^{2}$ \\
\hline Intercept & 0.112 \\
PR categories & \\
$41 \%-60 \%$ & 0.000 \\
$61 \%-100 \%$ & 0.324 \\
Interaction terms & \\
ADP category $2 *$ Intermediate risk level & 0.134 \\
ADP category $3 *$ Intermediate risk level & 0.086 \\
ADP category $2 *$ High risk level & 0.043 \\
ADP category $3 *$ High risk level & 0.002 \\
\hline
\end{tabular}

\section{Detailed results on studies using ADP $5 \mu \mathrm{M}$ to assess platelet reactivity}

Similarly to results found using $20 \mu \mathrm{M}$ ADP, for patients with none of the four risk factors described above, there was no increased risk for any of the PR strata $(H R=0.74$ [0.27;2.01], $\mathrm{p}=0.56$ for $\mathrm{PR}=31 \%-50 \%$; $\mathrm{HR}=1.20$ [0.42;3.47], $\mathrm{p}=0.73$ for $\mathrm{PR}=51 \%-100 \%$ ). In intermediate risk patients (one risk factor), there was an increased risk for both the medium and high strata of $\mathrm{PR}(\mathrm{HR}=2.87[1.40 ; 5.90]$ and $\mathrm{p}=0.004$ for $\mathrm{ADP} 5 \mu \mathrm{M} 31 \%-50 \% ; \mathrm{HR}=4.81$ 
[2.29;10.10] and $\mathrm{p}<0.0001$ for ADP $5 \mu \mathrm{M} 51 \%-100 \%$ ), while in high risk patients (two or more risk factors), the direction of the effect was the same but only significant for the high PR category $(H R=1.73$ [0.91;3.28] and $p=0.10$ for ADP $5 \mu M \quad 31 \%-50 \%$; $H R=2.84$ [1.49;5.43]

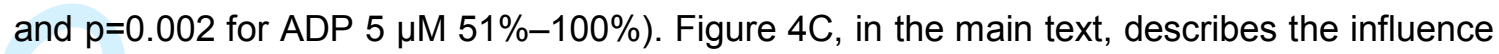
of PR, analysed as a continuous variable, on the two-year risk of MACE for the different patient risk levels. In low-risk patients, the MACE-free survival fluctuates between $90 \%$ and $95 \%$ with no pattern of decreased survival with higher PR. Intermediate risk patients have a reduced MACE-free survival corresponding to an increased risk of MACE for PR values above $30 \%$, while the risk of MACE in high-risk patients increases earlier (for PR values above $20 \%$ ). 
2
Supplemental Figure 3. Interaction between $5 \mu$ M ADP LTA values and the level of risk Low-risk patients have none of the risk factors (among age $>75$ years, acute coronary syndrome at inclusion, diabetes, and hypertension), intermediate-risk patients have one risk factor and high-risk patients have two or more risk factors.
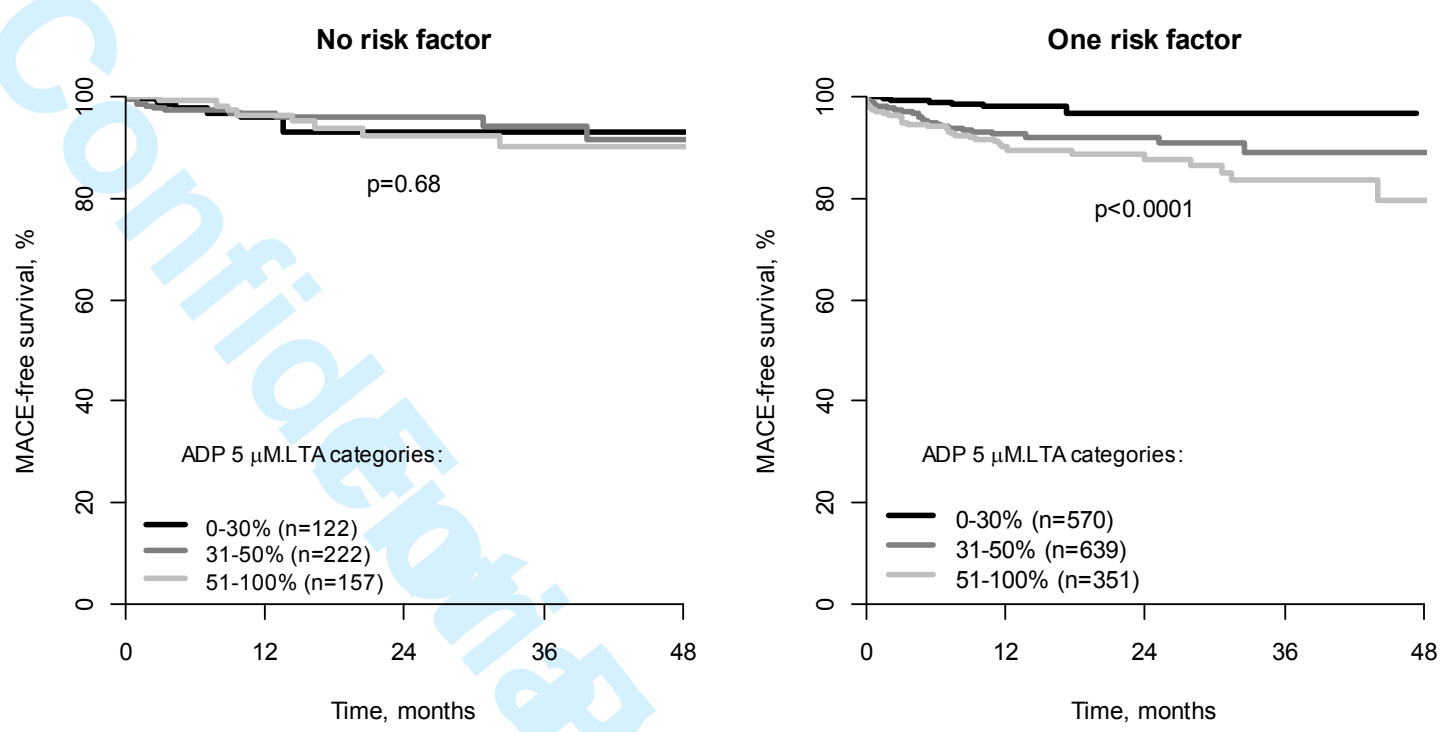


\section{Sensitivity analyses}

Sensitivity analyses were performed for PR using ADP $20 \mu \mathrm{M}$. A leave-one-out approach was applied to check the robustness of the association between PR using ADP $20 \mu \mathrm{M}$ and risk of MACE. Whichever study was removed, the association remained significant. The HR for the intermediate category of maximal aggregation using ADP $20 \mu \mathrm{M}(41 \%-60 \%)$ ranged from 1.64 (when Gurbel et al.'s study was removed) to 2.04 (when Angiolillo et al.'s study was removed). The HR for the higher category of maximal aggregation values $(61 \%-100 \%)$ ranged from 2.34 (when Gurbel et al.'s study was removed) to 3.29 (when Hochholzer et al.'s study was removed). Thus the association detected in the meta-analyses was not caused by any single study (Table1).

The leave-one-out approach was also applied to evaluate the robustness of the interaction between the number of risk factors and the level of PR in predicting MACE outcomes. The interaction remained at the same magnitude, but depending on which study was removed it was sometimes no longer significant (Table 1). This was most marked when Reny et al.'s study was removed, leading to a p-value of 0.22 for that interaction. In this particular case the magnitude of the non-significant interaction remained the same (Table 2).

In the studies evaluating PR using ADP $20 \mu \mathrm{M}$, two studies had an unclear risk of bias: Geisler et al.'s study, in the domain of 'flow and timing' due to patients lost to follow-up; ${ }^{31}$ and Campo et al.'s study, because adjudication of the outcome was not stated as blinded to the PR test results, and because stroke and vascular death were not part of the composite MACE outcome. ${ }^{27}$ When these two studies were removed, the results on the interaction with risk factors were similar (Table 2). There were unclear concerns about applicability of Angiolillo et al.'s study as it included diabetic patients exclusively. ${ }^{29}$ Removing this study did not affect the interaction with risk factors.

For the main analysis, target vessel revascularisation (TVR) was not included in the composite MACE outcome; however, four studies had TVR information available. A reanalysis of data, restricted to these four studies and comprising 1,066 patients, was performed with a definition of MACE including TVR $(n=160)$. The results were similar to those obtained when TVR was not included in the composite MACE outcome: the adjusted HRs were $2.92[1.55 ; 5.51](p=0.0009)$ and $4.98[1.72 ; 14.43](p=0.003)$ for the intermediate and high categories of $P R$, respectively. When restricted to the population of 3,564 patients treated with $\mathrm{PCl}$ and tested using $20 \mu \mathrm{M}$ ADP, the interaction was of similar magnitude but no longer significant (Table 3). Similarly, the interactions with the number of risk factors 
(Table 3) remained of the same magnitude (compared to the main analysis of nine studies on 4,438 patients), but were no longer significant ( $p=0.25)$.

The robustness of the findings with regard of the choice of cut-offs for PR evaluated using ADP $20 \mu \mathrm{M}$ was checked. Categories of PR were determined by the quartile of PR: $0 \%-$ $38.1 \%, 38.2 \%-51.3 \%, 51.4 \%-63.0 \%, 63.1-100 \%$. In low-risk patients (no risk factors), the HRs for categories $38.2 \%-51.3 \%, 51.4 \%-63.0 \%$, and $63.1-100 \%$ were respectively 0.45 $[0.12 ; 1.68] \quad(p=0.23), \quad 0.88 \quad[0.29 ; 2.71] \quad(p=0.82)$, and $1.98 \quad[0.61 ; 6.48] \quad(p=0.24)$. In intermediate-risk patients (one risk factor), the HRs were $1.33[0.64 ; 2.72](p=0.44), 1.54$ [0.77;3.09] $(p=0.22)$, and $4.73[2.17 ; 10.31](p<0.0001)$. In high-risk patients (two or more risk factors), the HRs were $2.64[1.35 ; 5.17](p=0.005), 3.58[1.90 ; 6.75](p<0.0001)$, and 4.21 [1.96;9.05] $(p=0.0002)$. The interaction between the level of cardiovascular risk and PR was statistically significant $(p=0.01)$.

An additional sensitivity analysis was carried out to check the robustness of the main findings with a different categorization of the level of risk. Alternate choices were restricted for different reasons : i) the increase in the risk of MACE is aleardy present in patients with one risk factor compared to those with none $(H R=1.61$ [1.05;2.45], $p=0.03$, as shown in table 2 of the manuscript) thus precluding the grouping of patients with 0 or 1 risk factor; ii) patients with 4 risk factors had the highest risk of MACE. However, the size of this sub-group $(n=173)$ was much too small to analyze it as a single category of risk level. We therefore performed a sensitivity analysis with four risk levels : first level $=0$ risk factor, second level $=1$ risk factor, third level $=2$ risk factors, fourth levels $=3$ or 4 risk factors. The magnitude of the interaction between risk level and PR level was similar between this categorization with four risk levels and the categorization with three risk levels shown in the manuscript. However the interaction term was not significant with this new categorization $(p=0.11$ from the mixed effect Cox model). This can logically be explained by the loss of power due to the higher number of parameters involved with the additional risk category. Detailed results are shown in the tables below

Number of patients according to the number of risk factors in studies with PR measured by $20 \mu \mathrm{M}$ ADP, $10 \mu \mathrm{M}$ ADP and $5 \mu \mathrm{M}$ ADP (after exclusion of patients with missing data for PR).

\begin{tabular}{lccc} 
& $\mathbf{2 0} \boldsymbol{\mu M}$ ADP & $\mathbf{1 0} \boldsymbol{\mu M}$ ADP & $\mathbf{5} \boldsymbol{\mu M}$ ADP \\
\hline No RF & 554 & 5 & 519 \\
$\mathbf{1}$ RF & 1874 & 520 & 1564 \\
$\mathbf{2}$ RFs & 1371 & 856 & 934 \\
$\mathbf{3}$ RFs & 455 & 604 & 189 \\
$\mathbf{4}$ RFs & 85 & 97 & 13 \\
\hline
\end{tabular}


HRs for MACE accrording to PR within each risk level.

\begin{tabular}{|c|c|c|c|c|c|}
\hline & \multicolumn{5}{|c|}{$20 \mu \mathrm{M}$ ADP } \\
\hline & $0-40$ & $41-60$ & & $61-100$ & \\
\hline Risk & & & & & \\
\hline level & $H R$ & $\mathrm{HR}[95 \% \mathrm{Cl}]$ & $p$ value & $\mathrm{HR}[95 \% \mathrm{Cl}]$ & $p$ value \\
\hline No RF & 1 (ref) & $0.96[0.33 ; 2.79]$ & 0.95 & $2.15[0.68 ; 6.82]$ & 0.19 \\
\hline $1 \mathrm{RF}$ & 1 (ref) & $1.20[0.66 ; 2.18]$ & 0.55 & 3.19 [1.59;6.41] & 0.001 \\
\hline $2 R F s$ & 1 (ref) & $2.91[1.42 ; 5.97]$ & 0.004 & $3.87[1.70 ; 8.81]$ & 0.001 \\
\hline 3, 4 RFs & 1 (ref) & $2.99[1.12 ; 7.97]$ & 0.03 & $3.31[1.15 ; 9.54]$ & 0.03 \\
\hline
\end{tabular}


Supplemental Table 1. Sensitivity analysis for the association between PR using $20 \mu M$ ADP and risk of MACE (leave-oneout procedure)

\begin{tabular}{|c|c|c|c|c|c|c|}
\hline \multirow[b]{2}{*}{ Removed study } & \multirow[b]{2}{*}{$\mathrm{N}$ analysed / $\mathrm{N}$ events } & \multicolumn{2}{|c|}{$20 \mu \mathrm{M}$ ADP LTA 41\%-60\% } & \multicolumn{2}{|c|}{$20 \mu \mathrm{M}$ ADP LTA 61\%-100\% } & \multirow{2}{*}{$\begin{array}{c}\text { Interaction between PR } \\
\text { and risk level } \\
\mathrm{p} \\
\end{array}$} \\
\hline & & $\mathrm{HR}[95 \% \mathrm{Cl}]$ & $\mathrm{p}$ & $\mathrm{HR}[95 \% \mathrm{Cl}]$ & $p$ & \\
\hline Hochholzer et al. & $3375 / 267$ & $1.98[1.29 ; 3.04]$ & 0.002 & $3.29[2.00 ; 5.43]$ & $<0.0001$ & 0.08 \\
\hline Angiolillo et al. & $3967 / 251$ & $2.04[1.35 ; 3.10]$ & 0.0008 & $2.93[1.74 ; 4.94]$ & $<0.0001$ & 0.02 \\
\hline Campo et al. & $4075 / 283$ & $1.77[1.20 ; 2.62]$ & 0.004 & $2.57[1.60 ; 4.12]$ & $<0.0001$ & 0.04 \\
\hline Cuisset et al. 2007 & $3951 / 279$ & $1.81[1.23 ; 2.67]$ & 0.003 & $2.81[1.67 ; 4.73]$ & $<0.0001$ & 0.08 \\
\hline Gurbel et al. & $3882 / 252$ & $1.61[1.07 ; 2.41]$ & 0.02 & $2.31[1.46 ; 3.66]$ & 0.0004 & 0.08 \\
\hline Breet et al. & $3089 / 197$ & $1.75[1.15 ; 2.67]$ & 0.009 & $2.89[1.61 ; 5.18]$ & 0.0004 & 0.03 \\
\hline Yong et al. & $3953 / 273$ & $1.84[1.24 ; 2.75]$ & 0.003 & $3.06[1.85 ; 5.06]$ & $<0.0001$ & 0.07 \\
\hline
\end{tabular}


Supplemental Table 2. Results of the sensitivity analysis for the modification of the association between PR, assessed with $20 \mu \mathrm{M}$ ADP and risk of MACE

\begin{tabular}{|c|c|c|c|c|c|c|c|c|c|c|}
\hline & \multicolumn{2}{|c|}{ Reny study removed } & \multicolumn{2}{|c|}{ Geisler study removed } & \multicolumn{2}{|c|}{ Campo study removed } & \multicolumn{2}{|c|}{$\begin{array}{l}\text { Geisler \& Campo } \\
\text { studies removed }\end{array}$} & \multicolumn{2}{|c|}{ Angiolillo study removed } \\
\hline & $\mathrm{HR}[95 \% \mathrm{Cl}]$ & $\mathrm{p}$ & $\mathrm{HR}[95 \% \mathrm{Cl}]$ & $\mathrm{p}$ & $\mathrm{HR}[95 \% \mathrm{Cl}]$ & $\mathrm{p}$ & $\mathrm{HR}[95 \% \mathrm{Cl}]$ & $\mathrm{p}$ & $\mathrm{HR}[95 \% \mathrm{Cl}]$ & $\mathrm{p}$ \\
\hline \multicolumn{11}{|l|}{$\begin{array}{l}\text { Interaction with } \\
\mathrm{Nb} \text { Risk factors }\end{array}$} \\
\hline \multicolumn{11}{|l|}{ No risk factors } \\
\hline ADP $200 \%-40 \%$ & Ref & & Ref & & Ref & & Ref & & Ref & \\
\hline ADP 20 41\%-60\% & $1.26[0.31 ; 5.03]$ & 0.75 & $0.94[0.32 ; 2.71]$ & 0.90 & $0.96[0.33 ; 2.78]$ & 0.95 & $0.93[0.32 ; 2.69]$ & 0.90 & $0.96[0.33 ; 2 . .78]$ & 0.94 \\
\hline ADP 20 61\%-100\% & $2.23[0.50 ; 9.72]$ & 0.29 & $2.05[0.60 ; 6.93]$ & 0.25 & $1.88[0.61 ; 5.77]$ & 0.27 & $1.68[0.52 ; 5.36]$ & 0.38 & $1.98[0.62 ; 6.35]$ & 0.25 \\
\hline \multicolumn{11}{|l|}{ One risk factor } \\
\hline ADP $200 \%-40 \%$ & Ref & & Ref & & Ref & & Ref & & Ref & \\
\hline ADP 20 41\%-60\% & $1.43[0.74 ; 2.77]$ & 0.28 & $1.45[0.75 ; 2.83]$ & 0.27 & $1.16[0.64 ; 2.11]$ & 0.62 & $1.40[0.72 ; 2.72]$ & 0.33 & $1.47[1.01 ; 2.14]$ & 0.54 \\
\hline $\begin{array}{l}\text { ADP } 2061 \%-100 \% \\
\text { Two or more risk } \\
\text { factors }\end{array}$ & $4.03[1.89 ; 8.63]$ & 0.0003 & $4.24[1.85 ; 9.69]$ & 0.0006 & $2.77[1 . .44 ; 5.31]$ & 0.002 & $3.43[1.63 ; 7.24]$ & 0.001 & $3.02[1.45 ; 6.26]$ & 0.003 \\
\hline ADP $200 \%-40 \%$ & Ref & & Ref & & Ref & & Ref & & Ref & \\
\hline ADP 20 41\%-60\% & $2.47[1.36 ; 4.49]$ & 0.003 & $3.04[1.54 ; 6.00]$ & 0.001 & $2.82[1.55 ; 5.13]$ & 0.0007 & $2.96[1.46 ; 6.00]$ & 0.003 & $3.85[1.95 ; 7.58]$ & $<0.0001$ \\
\hline ADP 20 61\%-100\% & $3.54[1.71 ; 7.31]$ & 0.0006 & $4.01[1.71 ; 9.38]$ & 0.001 & $3.15[1.60 ; 6.19]$ & 0.0009 & $3.20[1.43 ; 7.14]$ & 0.005 & $4.47[1.97 ; 10.16]$ & 0.0003 \\
\hline Interaction (p) & & 0.22 & & 0.04 & & 0.04 & & 0.04 & & 0.02 \\
\hline
\end{tabular}


Supplemental Table 3. Results of the sensitivity analysis for the modification of the association between PR using $20 \mu M$ ADP and risk of MACE in patients with PCl at inclusion and when target vessel revascularisation (TVR) is included in the composite outcome of MACE.

\begin{tabular}{|c|c|c|c|c|}
\hline \multirow[b]{2}{*}{$\mathrm{N}$ patients } & \multicolumn{2}{|c|}{ Patients with $\mathrm{PCl}$} & \multicolumn{2}{|c|}{ MACE event definition incl. TVR } \\
\hline & \multicolumn{2}{|l|}{3,406} & \multicolumn{2}{|l|}{1,066} \\
\hline \multirow[t]{2}{*}{$\mathrm{N}$ events } & \multicolumn{2}{|l|}{198} & \multicolumn{2}{|l|}{160} \\
\hline & $\mathrm{HR}[95 \% \mathrm{Cl}]$ & $\mathrm{p}$ & $\mathrm{HR}[95 \% \mathrm{Cl}]$ & $\mathrm{p}$ \\
\hline \multicolumn{5}{|l|}{ Interaction with } \\
\hline \multicolumn{5}{|l|}{ Nb Risk factors } \\
\hline \multicolumn{5}{|l|}{ No risk factors } \\
\hline ADP 20 0\%-40\% & Ref & & Ref & \\
\hline ADP 20 41\%-60\% & $1.26[0.31 ; 5.04]$ & 0.75 & $1.43[0.69 ; 5.04]$ & 0.58 \\
\hline ADP 20 61\%-100\% & $2.32[0.51 ; 10.49]$ & 0.27 & $3.41[0.67 ; 17.41]$ & 0.14 \\
\hline \multicolumn{5}{|l|}{ One risk factor } \\
\hline ADP 20 0\%-40\% & Ref & & Ref & \\
\hline ADP 20 41\%-60\% & $1.44[0.72 ; 2.89]$ & 0.3 & $2.24[0.73 ; 6.89]$ & 0.16 \\
\hline ADP 20 61\%-100\% & $4.16[1.78 ; 9.72]$ & 0.001 & $7.52[1.86 ; 30.37]$ & 0.005 \\
\hline \multicolumn{5}{|c|}{ Two or more risk factors } \\
\hline ADP $200 \%-40 \%$ & Ref & & Ref & \\
\hline ADP 20 41\%-60\% & $3.36[1.61 ; 6.8]$ & 0.001 & $4.07[1.79 ; 9.28]$ & 0.0008 \\
\hline ADP 20 61\%-100\% & $4.97[2.03 ; 12.16]$ & 0.0004 & $6.14[1.82 ; 20.65]$ & 0.003 \\
\hline Interaction $(p)$ & & 0.21 & & 0.25 \\
\hline
\end{tabular}




\section{Supplemental Figure 4. Funnel plot for detection of a potential publication bias.}

The hazard ratio for the association between PR using $20 \mu \mathrm{M}$ ADP (per 10\%) and the risk of MACE was assessed in each study with adjustment on risk factors. The logarithm of the hazard ratios and their standard errors were represented in the funnel plot (white circles). The white diamond shows the pooled hazard ratio $(1.25,95 \% \mathrm{Cl} 1.08$ to 1.44$)$. Two studies were detected as missing using the 'trim and fill' method for the funnel plot to be symmetrical (black circles). However, when these missing studies were added, the pooled hazard ratio was not significantly modified $(1.21,95 \% \mathrm{Cl} 1.05$ to 1.40$)$.

\section{Funnel Plot of Standard Error by Point estimate}

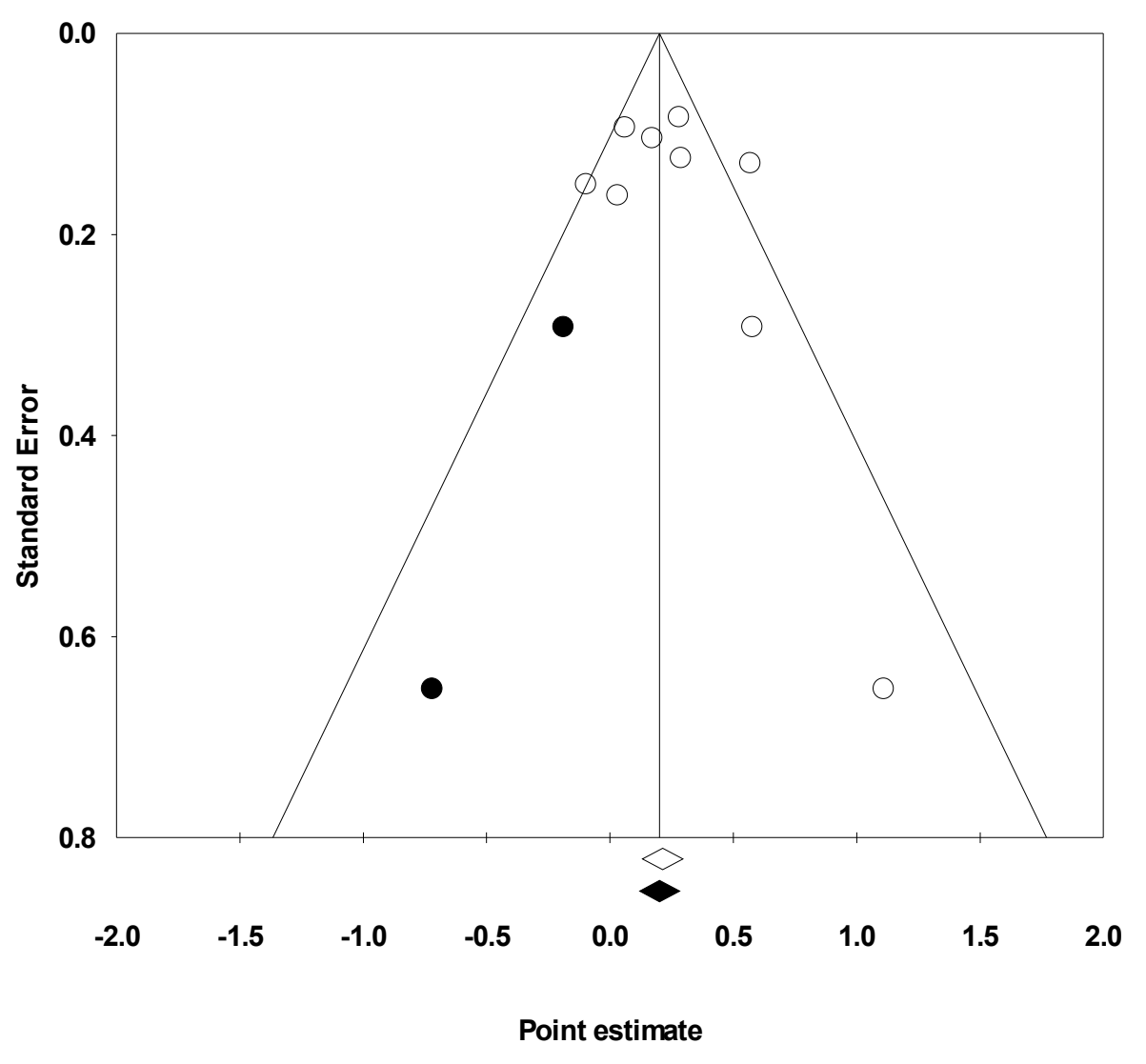

49

50

51

52

53

54

55

56 


\section{References}

1. Eikelboom JW, Hirsh J, Spencer FA, et al. Antiplatelet drugs: Antithrombotic Therapy and Prevention of Thrombosis, 9th ed: American College of Chest Physicians EvidenceBased Clinical Practice Guidelines. Chest 2012;141(2 Suppl):e89S-119S.

2. Yusuf S, Zhao F, Mehta SR, et al. Effects of clopidogrel in addition to aspirin in patients with acute coronary syndromes without ST-segment elevation. N Engl J Med $2001 ; 345(7): 494-502$.

3. Gurbel PA, Bliden KP, Hiatt BL, et al. Clopidogrel for coronary stenting: response variability, drug resistance, and the effect of pretreatment platelet reactivity. Circulation 2003;107(23):2908-13.

4. Hochholzer W, Trenk D, Frundi D, et al. Time dependence of platelet inhibition after a 600mg loading dose of clopidogrel in a large, unselected cohort of candidates for percutaneous coronary intervention. Circulation 2005;111(20):2560-4.

5. Snoep JD, Hovens MM, Eikenboom JC, et al. Association of laboratory-defined aspirin resistance with a higher risk of recurrent cardiovascular events: a systematic review and meta-analysis. Arch Intern Med 2007;167(15):1593-9.

6. Aradi D, Komocsi A, Vorobcsuk A, et al. Prognostic significance of high on-clopidogrel platelet reactivity after percutaneous coronary intervention: systematic review and meta-analysis. Am Heart J 2010;160(3):543-51.

7. Combescure C, Fontana P, Mallouk N, et al. Clinical implications of clopidogrel nonresponse in cardiovascular patients: a systematic review and meta-analysis. J Thromb Haemost 2010;8(5):923-33.

8. Reny JL, Berdague P, Poncet A, et al. Antiplatelet Drug Response Status Does Not Predict Recurrent Ischemic Events in Stable Cardiovascular Patients: Results of the Antiplatelet Drug Resistances and Ischemic Events Study. Circulation 2012;125(25):3201-10. 
9. Pettersen AA, Seljeflot I, Abdelnoor M, et al. High On-Aspirin Platelet Reactivity and Clinical Outcome in Patients With Stable Coronary Artery Disease: Results From ASCET (Aspirin Nonresponsiveness and Clopidogrel Endpoint Trial). Journal of the American Heart Association 2012;1(3):e000703.

10. Gurbel PA, Erlinge D, Ohman EM, et al. Platelet function during extended prasugrel and clopidogrel therapy for patients with ACS treated without revascularization: the TRILOGY ACS platelet function substudy. JAMA 2012;308(17):1785-94.

11. Bonello L, Camoin-Jau L, Arques S, et al. Adjusted clopidogrel loading doses according to vasodilator-stimulated phosphoprotein phosphorylation index decrease rate of major adverse cardiovascular events in patients with clopidogrel resistance: a multicenter randomized prospective study. J Am Coll Cardiol 2008;51(14):1404-11.

12. Bonello L, Camoin-Jau L, Armero S, et al. Tailored clopidogrel loading dose according to platelet reactivity monitoring to prevent acute and subacute stent thrombosis. Am J Cardiol 2009;103(1):5-10.

13. Price MJ, Berger PB, Teirstein PS, et al. Standard- vs high-dose clopidogrel based on platelet function testing after percutaneous coronary intervention: the GRAVITAS randomized trial. JAMA 2011;305(11):1097-105.

14. Collet JP, Cuisset T, Range G, et al. Bedside monitoring to adjust antiplatelet therapy for coronary stenting. N Engl J Med 2012;367(22):2100-9.

15. Aradi D, Komocsi A, Price MJ, et al. Efficacy and safety of intensified antiplatelet therapy on the basis of platelet reactivity testing in patients after percutaneous coronary intervention: systematic review and meta-analysis. Int J Cardiol 2013;167(5):2140-8.

16. Stone GW, Witzenbichler B, Weisz G, et al. Platelet reactivity and clinical outcomes after coronary artery implantation of drug-eluting stents (ADAPT-DES): a prospective multicentre registry study. Lancet 2013;382(9892):614-23.

17. Brar SS, ten Berg J, Marcucci R, et al. Impact of platelet reactivity on clinical outcomes after percutaneous coronary intervention. A collaborative meta-analysis of individual participant data. J Am Coll Cardiol 2011;58(19):1945-54. 
18. Yamaguchi $Y$, Abe $T$, Sato $Y$, et al. Effects of VerifyNow P2Y12 test and CYP2C19*2 testing on clinical outcomes of patients with cardiovascular disease: a systematic review and meta-analysis. Platelets 2013;24(5):352-61.

19. Moher D, Pham B, Klassen TP, et al. What contributions do languages other than English make on the results of meta-analyses? J Clin Epidemiol 2000;53(9):964-72.

20. Berlin JA. Does blinding of readers affect the results of meta-analyses? University of Pennsylvania Meta-analysis Blinding Study Group. Lancet 1997;350(9072):185-6.

21. Bonello L, Tantry US, Marcucci R, et al. Consensus and future directions on the definition of high on-treatment platelet reactivity to adenosine diphosphate. J Am Coll Cardiol 2010;56(12):919-33.

22. Tantry US, Bonello L, Aradi D, et al. Consensus and update on the definition of ontreatment platelet reactivity to adenosine diphosphate associated with ischemia and bleeding. J Am Coll Cardiol 2013;62(24):2261-73.

23. Stewart GB, Altman DG, Askie LM, et al. Statistical analysis of individual participant data meta-analyses: a comparison of methods and recommendations for practice. PLoS One 2012;7(10):e46042.

24. Akritas MG. Nearest neighbor estimation of a bivariate distribution under random censoring. Ann Stat 1994;22:1299-327.

25. Pencina MJ, D'Agostino RB, Sr., Steyerberg EW. Extensions of net reclassification improvement calculations to measure usefulness of new biomarkers. Stat Med 2011;30(1):11-21.

26. Stroup DF, Berlin JA, Morton SC, et al. Meta-analysis of observational studies in epidemiology: a proposal for reporting. Meta-analysis Of Observational Studies in Epidemiology (MOOSE) group. JAMA 2000;283(15):2008-12.

27. Campo G, Valgimigli M, Gemmati D, et al. Value of Platelet Reactivity in Predicting Response to Treatment and Clinical Outcome in Patients Undergoing Primary Coronary Intervention. Insights Into the STRATEGY Study. Journal of the American College of Cardiology 2006;48(11):2178-85. 
28. Hochholzer W, Trenk D, Bestehorn HP, et al. Impact of the degree of peri-interventional platelet inhibition after loading with clopidogrel on early clinical outcome of elective coronary stent placement. J Am Coll Cardiol 2006;48(9):1742-50.

29. Angiolillo DJ, Bernardo E, Sabate M, et al. Impact of platelet reactivity on cardiovascular outcomes in patients with type 2 diabetes mellitus and coronary artery disease. J Am Coll Cardiol 2007;50(16):1541-7.

30. Cuisset T, Frere C, Quilici J, et al. High post-treatment platelet reactivity is associated with a high incidence of myonecrosis after stenting for non-ST elevation acute coronary syndromes. Thrombosis and haemostasis $2007 ; 97(2): 282-87$.

31. Geisler T, Grass D, Bigalke B, et al. The Residual Platelet Aggregation after Deployment of Intracoronary Stent (PREDICT) score. J Thromb Haemost 2008;6(1):54-61.

32. Gurbel PA, Antonino MJ, Bliden KP, et al. Platelet reactivity to adenosine diphosphate and long-term ischemic event occurrence following percutaneous coronary intervention: a potential antiplatelet therapeutic target. Platelets 2008;19(8):595-604.

33. Cuisset T, Frere C, Quilici J, et al. Predictive values of post-treatment adenosine diphosphate-induced aggregation and vasodilator-stimulated phosphoprotein index for stent thrombosis after acute coronary syndrome in clopidogrel-treated patients. Am J Cardiol 2009;104(8):1078-82.

34. Yong G, Rankin J, Ferguson L, et al. Randomized trial comparing 600- with 300-mg loading dose of clopidogrel in patients with non-ST elevation acute coronary syndrome undergoing percutaneous coronary intervention: results of the Platelet Responsiveness to Aspirin and Clopidogrel and Troponin Increment after Coronary intervention in Acute coronary Lesions (PRACTICAL) Trial. Am Heart J 2009;157(1):60 e1-9.

35. Breet NJ, van Werkum JW, Bouman $\mathrm{HJ}$, et al. Comparison of platelet function tests in predicting clinical outcome in patients undergoing coronary stent implantation. JAMA 2010;303(8):754-62. 
36. Marcucci R, Gori AM, Paniccia R, et al. High on-treatment platelet reactivity by more than one agonist predicts 12-month follow-up cardiovascular death and non-fatal myocardial infarction in acute coronary syndrome patients receiving coronary stenting. Thromb Haemost 2010;104(2):279-86.

37. Beigel $R$, Hod H, Fefer $P$, et al. Relation of aspirin failure to clinical outcome and to platelet response to aspirin in patients with acute myocardial infarction. Am J Cardiol 2011;107(3):339-42.

38. Aradi D, Rideg O, Vorobcsuk A, et al. Justification of $150 \mathrm{mg}$ clopidogrel in patients with high on-clopidogrel platelet reactivity. Eur J Clin Invest 2012;42(4):384-92.

39. Gagne JJ, Bykov K, Choudhry NK, et al. Effect of smoking on comparative efficacy of antiplatelet agents: systematic review, meta-analysis, and indirect comparison. BMJ 2013;347:f5307.

40. Lafitte M, Pucheu Y, Latry K, et al. Predictors of cardiovascular prognosis in patients receiving optimized secondary prevention measures after acute coronary syndrome. European journal of preventive cardiology 2013;20(2):283-90.

41. Cattaneo M. Aspirin and clopidogrel: efficacy, safety, and the issue of drug resistance. Arterioscler Thromb Vasc Biol 2004;24(11):1980-7.

42. Jeong YH, Bliden KP, Antonino MJ, et al. Usefulness of the VerifyNow P2Y12 assay to evaluate the antiplatelet effects of ticagrelor and clopidogrel therapies. Am Heart $\mathrm{J}$ 2012;164(1):35-42.

43. Van Werkum J, Van Der Stelt CA, Seesing TH, et al. A head-to-head comparison between the VerifyNow P2Y12 assay and light transmittance aggregometry for monitoring the individual platelet response to clopidogrel in patients undergoing elective percutaneous coronary intervention. J Thromb Haemost 2006;4(11):2516-8.

44. Coleman $\mathrm{Cl}$, Limone BL. Universal versus platelet reactivity assay-driven use of P2Y12 inhibitors in acute coronary syndrome patients: cost-effectiveness analyses for six European perspectives. Thromb Haemost 2014;111(1):103-10. 
45. Cayla G, Cuisset T, Silvain J, et al. Platelet function monitoring in elderly patients on prasugrel after stenting for an acute coronary syndrome: Design of the randomized antarctic study. American heart journal 2014;168(5):674-81.

46. Ripatti S, Palmgren J. Estimation of multivariate frailty models using penalized partial likelihood. Biometrics 2000;56(4):1016-22.

47. Therneau T, Grambsch P, Pankatz VS. Penalized survival models and frailty. J Computational and Graphical Statistics 2003;12:156-75.

48. Grambsch P, Therneau T. Proportional hazards tests and diagnostics based on weighted residuals. Biometrika 1994;81:515-26.

49. Duval S, Tweedie R. Trim and fill: A simple funnel-plot-based method of testing and adjusting for publication bias in meta-analysis. Biometrics 2000;56(2):455-63. 